\title{
Developments in the Field of Conducting and Non-conducting Polymer Based Potentiometric Membrane Sensors for Ions Over the Past Decade
}

\author{
Farnoush Faridbod ${ }^{1}$, Mohammad Reza Ganjali ${ }^{1, *}$, Rassoul Dinarvand ${ }^{2}$ and Parviz Norouzi ${ }^{1}$ \\ ${ }^{1}$ Center of Excellence in Electrochemistry, Faculty of Chemistry, University of Tehran, Tehran, Iran \\ ${ }^{2}$ Medical Nanotechnology Research Centre, Medical Sciences/University of Tehran, Tehran, P.O. Box \\ 14155-6451, Iran
}

* Author to whom correspondence should be addressed; E-mail: ganjali@khayam.ut.ac.ir; Tel: +98-21-61112788

Received: 1 February 2008 / Accepted: 2 April 2008 / Published: 3 April 2008

\begin{abstract}
Many research studies have been conducted on the use of conjugated polymers in the construction of chemical sensors including potentiometric, conductometric and amperometric sensors or biosensors over the last decade. The induction of conductivity on conjugated polymers by treating them with suitable oxidizing agents won Heeger, MacDiarmid and Shirakawa the 2000 Nobel Prize in Chemistry. Common conjugated polymers are poly(acetylene)s, poly(pyrrole)s, poly(thiophene)s, poly(terthiophene)s, poly(aniline)s, poly(fluorine)s, poly(3-alkylthiophene)s, polytetrathiafulvalenes, polynapthalenes, poly( $p$-phenylene sulfide), poly( $p$-phenylenevinylene $) \mathrm{s}$, poly(3,4-ethylenedioxythiophene), polyparaphenylene, polyazulene, polyparaphenylene sulfide, polycarbazole and polydiaminonaphthalene. More than 60 sensors for inorganic cations and anions with different characteristics based on conducting polymers have been reported. There have also been reports on the application of non-conducting polymers (nCPs), i.e. $\mathrm{PVC}$, in the construction of potentiometric membrane sensors for determination of more than 60 inorganic cations and anions. However, the leakage of ionophores from the membranes based on these polymers leads to relatively lower life times. In this article, we try to give an overview of Solid-Contact ISE (SCISE), Single-Piece ISE (SPISE), Conducting Polymer (CP)-Based, and also non-conducting polymer PVC-based ISEs for various ions which their difference is in the way of the polymer used with selective
\end{abstract}


membrane. In SCISEs and SPISEs, the plasticized PVC containing the ionophore and ionic additives govern the selectivity behavior of the electrode and the conducting polymer is responsible of ion-to-electron transducer. However, in CPISEs, the conducting polymer layer is doped with a suitable ionophore which enhances the ion selectivity of the $\mathrm{CP}$ while its redox response has to be suppressed.

Keywords: Conducting polymer, sensor, ion selective electrodes, non-conducting polymer, potentiometric membrane sensor

\section{Table of Contents}

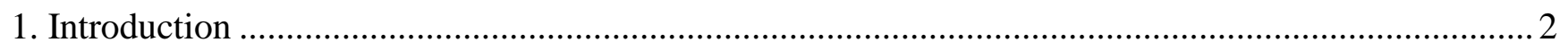

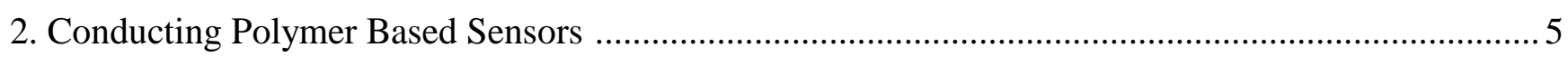

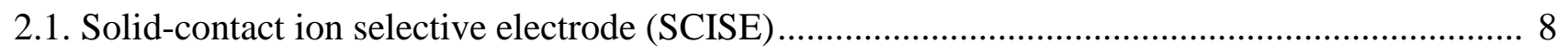

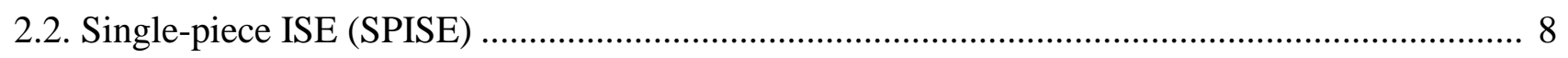

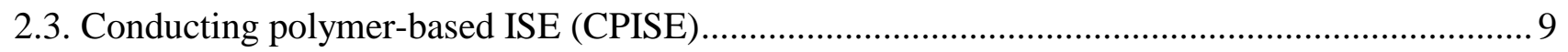

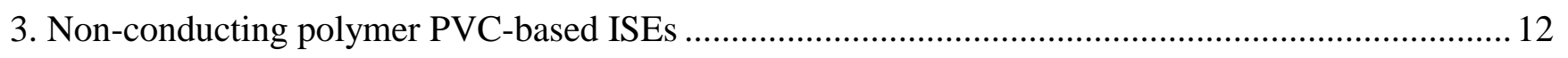

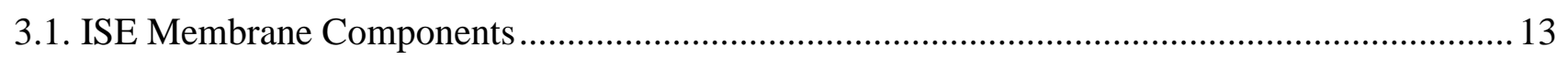

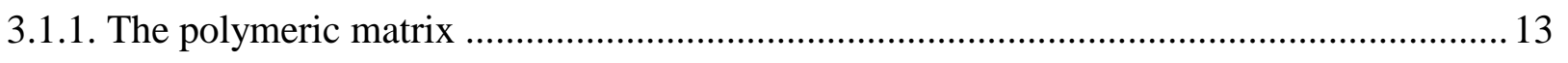

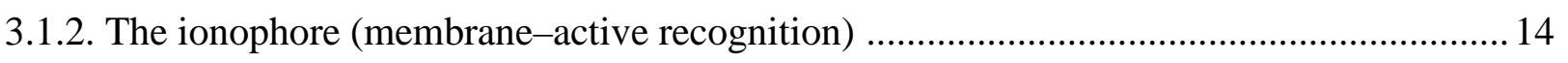

3.1.3. The membrane solvent (Plasticizer) ….................................................................... 15

3.1.4. Plasticizer-Free Polymer Membrane ISEs .......................................................... 16

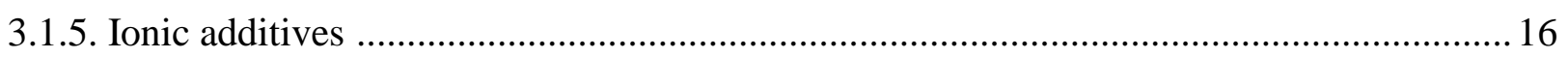

3.2. Non-conducting Polymer Membrane Sensors for the Hydrogen Ion and the First Main Group

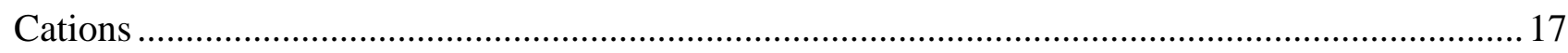

3.3. Non-conducting Polymer Membrane Sensors for the Second Main Group Cations .................. 19

3.4. Non-Conducting Polymer Membrane Sensors for the Third Main Group Cations .................. 21

3.5. Non-conducting Polymer Membrane Sensors for the Fourth Main Group Cations ................... 23

3.6. Non-conducting Polymer Membrane Sensors for the Transition Metal Cations....................... 26

3.7. Non-conducting Polymer Membrane Sensors for the Rare Earth Cations .............................. 31

3.8. Non-conducting Polymer Membrane Sensors for Inorganic Anions ...................................... 37

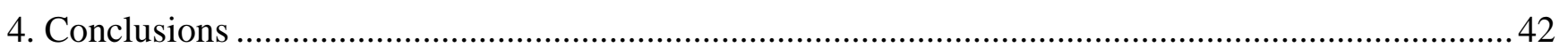

\section{Introduction}

Potentiometric measurements are based on monitoring potential values under a zero current flow regime, in order to determine the analytical concentration desired components in an analyte. In these methods the potential difference between an indicator electrode (i.e. ion-selective electrode, redox electrode, metal-metal oxide electrode) and the reference electrode is measured as the analytical potential. The potential of an electrochemical cell is due to the changes in the free energy that occurs if 
the chemical phenomena reach an equilibrium; which is a concept that is typically applied in quantitative analysis in relation to electrochemical cells, in which case, the difference between the cathodic and the anodic potentials is the potential of the electrochemical cell and is calculated using the so-called Nernst equation. Another source of potential may be physical phenomena not involving explicit redox reactions but having non-zero free energy initial conditions. For instance ion concentration gradients across a semi-permeable membrane can is one of such phenomena. This can also be potentiometric phenomena, and is the basis of measurements that use ion-selective electrodes, according to which the below equation is used to determine the concentration of the analyte:

$$
\mathrm{E}_{\mathrm{mem}}=\text { Constant }-\mathrm{RT} / \mathrm{z}_{\mathrm{i}} \mathrm{F} \ln \mathrm{a}_{\mathrm{i}}
$$

where $z_{i}$ and $a_{i}$ represent the charges and the activity of the ion of interest respectively. Potentiometric ion sensors represent well-established analytical tools. The ISE illustrated in Figure 1 is a typical electrode in which the transduction between the ion and electron takes place at the internal reference electrode $(\mathrm{Ag} / \mathrm{AgCl})$ immersed in the inner filling solution $(\mathrm{KCl})$, as follows:

$$
\mathrm{AgCl}+e^{-} \leftrightarrow A g+C l^{-}
$$

Figure 1. A classical ion-selective electrode ISE.

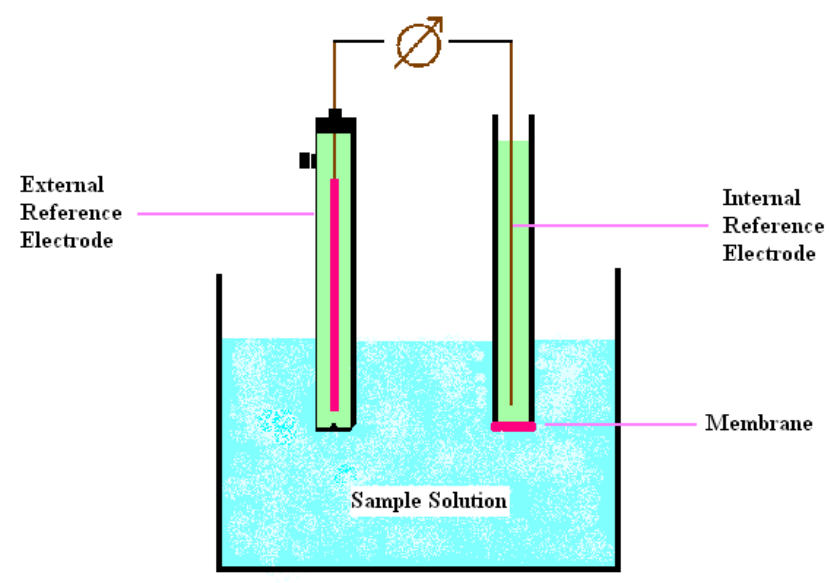

The identical reaction (2) ocurrs at the external reference electrode (RE), illustrating the reversible and stable connection between the ionic $\left(\mathrm{Cl}^{-}\right)$and electronic $\left(e^{-}\right)$signals. The selectivity is the result of incorporation of specific ionophores in the ion-selective membrane. Potentiometric ion sensors (ISEs) are very attractive, especially due to their small-size and portability, and low cost. Neutral or charged carrier based ISEs, which are available for about 60 different analytes, have found widespread applications in routine uses like clinical analysis [1-7]. On the other hand, to fully use the potentials of this technique, more durable and maintenance-free ISEs that are easily miniaturized are needed.

The works showing that the detection limit of conventional ISEs has the potentials to be lowered towards the sub-nanomolar concentration level [8], making them suitable for trace analyses has attracted more interest to these devices [2]. The development of new and applicable membranes such as plasticizer-free membranes [9], covalently attached ionophores [10], stable carborane anions [11], and monolithic capillary based ISEs [12], can be regarded as a sign of the great interest in these analytical tools. 
Fabrication of all-solid-state ISEs that do not require internal filling solutions is another common approach, which necessitates careful design of the solid contact between the ion-selective membrane and the electronic conductor [9-11, 13]. The introduction of the so-called coated-wire electrodes (Figure 2b) about three decades ago, can be regarded as an important step towards solid-state ISEs [14]. These devices, however, suffered from the ill-defined transduction of the ionically conducting ion-selective membrane and the electronic conductor, which made them rather instable. Scientists overcame this by simply using a hydrogel based electrolyte instead of the liquid internal electrolyte of the classical ISE (Figure 2a and 2c) [15]. Hydrogel-contact ISEs suffer, however, from shortcomings originating from the water uptake/release and the resulting volume changes of the hydrogel layer which is a function of the salt concentration of the hydrogel. Another approach to solid-state ISEs is based on the modification of the coated-wire electrodes (Figure 2b), through the application of an intermediate layer of suitable redox and ion-exchange properties at the wire membrane interface [13], in which case, even the application of a monolayer of redox-active compound allows a well-defined pathway for ionto-electron transduction [16]. Monolayers, however, suffer an inherently low frequency redox capacitance to provide stability condition which depends on the low frequency redox capacitance of the conducting polymer. The low-frequency impedance response of conducting polymers is similar to ideal capacitance resulting from reversible oxidation of the polymer bulk connected with ion transport. The small current that passes through the electrode during potentiometric measurements inevitably results in oxidation/reduction of the conducting polymer, i.e. charging/discharging of the bulk capacitance [17].

Figure 2. Structure of ion selective electrode electrodes; a) conventional ISE with an internal reference electrode and internal filling solution; b) coated wire or graphite coated ISE; c) ISE with a hydrogel contact.

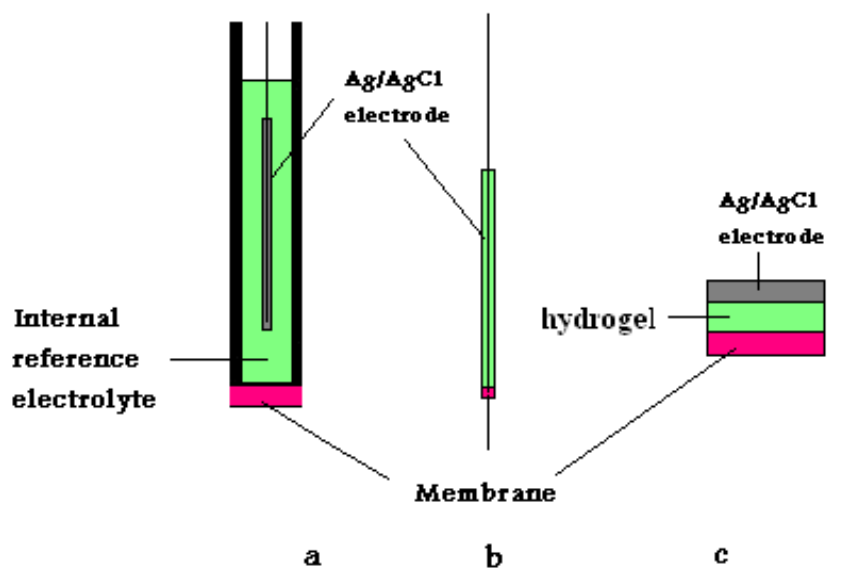

The above mentioned may be one of the reasons behind the increasing interest in the application of conducting polymers as ion-to-electron transducers in this group of ISEs [9-11]. The ion-to-electron transduction process in this case is quite identical with the mechanism of this process at the internal reference electrode of the conventional or hydrogel-contact ISE and can be summarized as:

$$
\mathrm{M}^{+} \mathrm{A}^{-}+\mathrm{e} \leftrightarrow \mathrm{M}+\mathrm{A}^{-}
$$


where $\mathrm{M}^{+}$is metal ion (e.g. $\mathrm{Ag}^{+}$) or oxidized conducting polymer unit, $\mathrm{e}^{-}$is electron, $\mathrm{M}$ is metal (e.g. $\mathrm{Ag}$ ) or neutral conducting polymer unit, and $\mathrm{A}^{-}$is anion (e.g. $\mathrm{Cl}^{-}$).

Because of the priceless electrical and electrochemical characteristics of conducting polymers, they have found many applications since their discovery. One of them is their wide use in the fabrication of electrochemical sensors such as potentiometric, amperometric and conductometric sensors [10, 18]. They can be used to convert chemical information such as concentration or activity into electrical signals in the solid state. Combination of the properties of the conjugated polymer transducers with those leading to selective molecular recognition is a very important step in the way of obtaining durable chemical sensors. This wide application and great interest is mostly due to the their potential to exhibit improved response properties and also their high sensitivity to small perturbations which are great advantages in comparison to the inert polymers were which used only to enhance the mechanical strength of the membranes. Apart from their unique conductivity and ion transport properties leading to the mentioned advantages, conducting polymers also enjoy the benefits of being compatible with biological molecules in neutral aqueous solutions or even they can be used to bind biomolecules to a biosensor [18-22].

\section{Conducting Polymer Based Sensors}

Detailed classifications divide "conducting polymers" into several types, including doped conjugated and redox polymers, polymer composites and polymer electrolytes. These compounds have shown to be very promising for the purpose of building different chemical sensors [23-25]. There are several reasons behind their being suitable as transducers in solid-state ISEs. First, they are able to form an ohmic contact to materials of high work functions, like carbon, gold and platinum. Second, due to the solubility of several CPs, they can be deposited from solutions. Third, they have ability to be electroactive materials of mixed electronic and ionic conductivity, which gives them the unique potential to transduce ionic signals into electronic ones.

Figure 3 illustrates some CP monomers and CPs commonly used as ion-to-electron transducers in construction of all solid state potentiometric sensors. Conducting polymers have been used in the construction of ISEs according to general approaches, which have led to the development of solid contact ion selective electrodes (SCISEs), and single-piece ion selective electrodes (SPISEs) and conducting polymer based ion selective electrodes (CPISEs). In SCISEs, the conducting polymer is electro polymerized on the surface of the electronic conductor and then, coated with the ion-selective membrane. The selective membrane determines the selectivity, and the conducting polymer only acts as an ion-to-electron transducer of the sensor. This way, both the ion-exchange and redox characteristics of the conducting polymers are successfully combined with the selectivity of the ionophore-based ion-selective membranes. In SPISEs, the selective membrane contains conducting polymer due to the solubility and the consequent dispersability of conjugated polymers in an organic solvent. In both the above mentioned ISEs, the plasticized PVC containing the ionophore and ionic additives govern the selectivity behavior of the electrode and the conducting polymer is responsible of ion-to-electron transducer. In CPISEs, the conducting polymer layer is doped with a suitable ionophore which enhances the ion selectivity of the $\mathrm{CP}$ while its redox response has to be suppressed. Thus, the 
redox sensitivity limits the use of CPISEs in direct measurements. The further explanation about these different types of ISEs will be discussed below.

Figure 3. Common used $\mathrm{CP}$ monomers and $\mathrm{CPs}$ as ion-to-electron transducers in construction of all solid state potentiometric sensors.

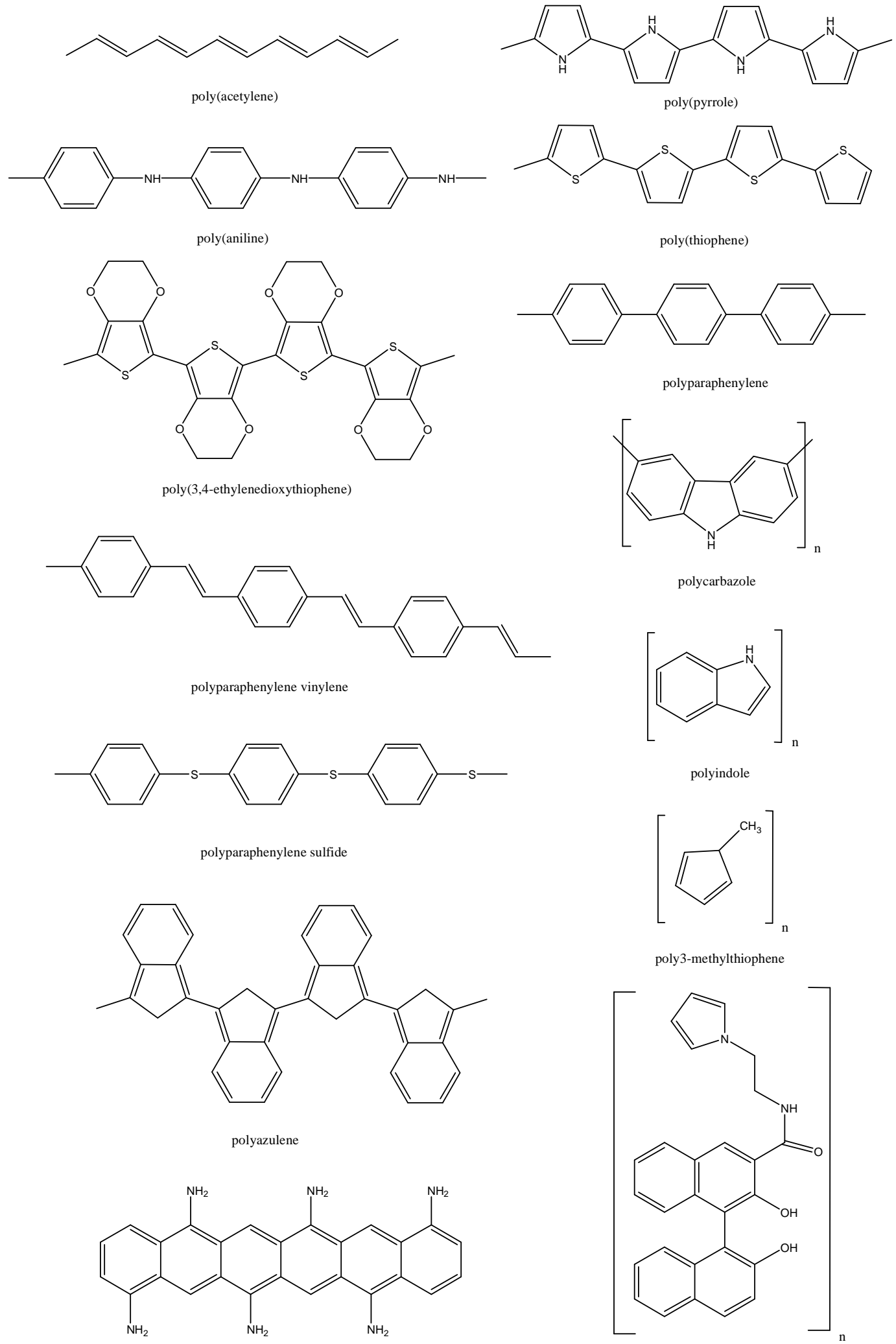

polydiaminonaphthalene 


\subsection{Solid-contact ion selective electrode (SCISE)}

To construct an SCISE (Figure 4) the CP can be electropolymerized on an electronic conductor and then coated with the ion-selective membrane. In the case of the electrodes prepared in this way, the PVC ion selective coating determines the ion selectivity, while the CP film acts as the ion-to-electron transducer. Bobacka et al. have recently used a number of CPs, like polypyrrole (PPy), poly(3-octylthiophene, POT), and poly(3,4-ethylenedioxythiophene, PEDOT) for the construction of SCISEs [911]. Due the special nature and design of the SCISEs, these devices have also been miniaturized to yield solid state ion-selective microelectrodes [26].

Figure 4. different types of conducting polymeric based ISE; a) electronic conductor; b)electronic conductor with a high work function (the work function is the minimum energy needed to remove an electron from a solid to a point close to the solid on the macroscopic scale outside the solid surface); c) conducting polymer; d) ion selective membrane; e) ion selective membrane containing conducting polymer; f) conducting polymer doped with ionophores.

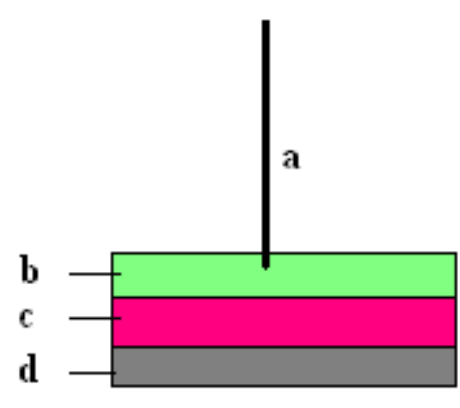

SCISE

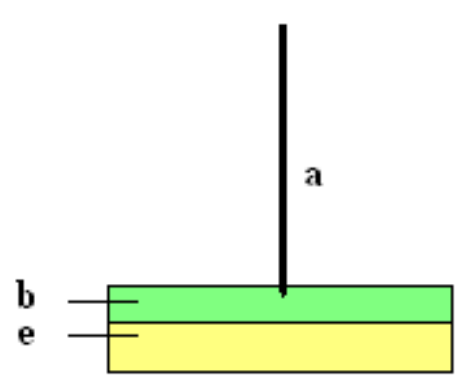

SPISE

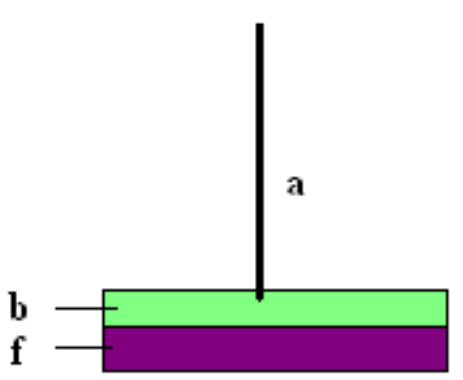

CPISE

\subsection{Single-piece ISE (SPISE)}

This approach uses the solubility, and the consequent dispersability, of conjugated polymers in organic solvents such as tetrahydrofuran (THF), which is a commonly used as the solvent to dissolve the membrane components during the preparation of plasticized PVC-based ISEs. So, in case the CP is also dissolved as an additional membrane component it can be physically integrated within the ionselective membrane, resulting in the so-called single-piece electrode (SPISE) [27]. The presence of the $\mathrm{CP}$ in the membrane composition, however, definitely influences the electrode selectivity which changes the role of the CP from SCISE where they have a mere physical role, to SPISEs (Figure 4) where the $\mathrm{CP}$ also has a role as a membrane composition, which necessitates the careful optimization of this species in the later approach. It should be noted that, no matter which of the two abovementioned approaches is used, it is still the plasticized PVC containing the ionophore and ionic additives that governs the selectivity behavior of the electrode.

On the other hand, since the life time of the plasticized PVC membranes is affected by leaching of the membrane components from the membrane into the sample solution, many efforts have been made 
to achieve plasticizer-free membranes and covalently bound ion recognition sites, in which case CPs also offer unique possibilities.

\subsection{Conducting polymer-based ISE(CPISE)}

Conducting polymers (e.g. PPY) are known to possess mixed ionic/redox responses, which introduces some considerations, in case one tends to develop ISEs with a CP polymeric matrix or in other words a conducting polymer-based ISE (CPISE), including the fact that the ion selectivity of the $\mathrm{CP}$ should be enhanced while its redox response has to be suppressed. This can be achieved through doping the CP with metal-complexing ligands, the ion selectivity of which depends on the metalcomplexing ligand used [28]. Although the redox sensitivity limits the use of CPISEs in direct measurements, such ISEs have been successfully used as potentiometric indicator electrodes in nonclassical titrations [29]. For instance in the case of the PPy electrodes, the applicability of the CPISE in titrations was demonstrated by determination of $\mathrm{Ca}^{2+}$ in mineral water [30].

In another attempt to construct a CPISE valinomycin and a lipophilic anion were incorporated in undoped POT to build $\mathrm{K}^{+}$selective ISEs by dissolving the ingredients in chloroform, and casting the CPISE membrane [31], resulting semi-conducting organic polymer with a reduced redox response (compared to PPy) due to the low electronic conductivity of the undoped POT and the high $\mathrm{K}^{+}$selectivity of valinomycin. This is an indication that the selectivity of CP-based membranes can be greatly enhanced by addition of suitable ionophores and ionic sites [31]. POT has also been used, in a similar way, to prepare $\mathrm{Cl}^{-}$sensors using tridodecylmethylammonium chloride (TDMACl) [32]. $\mathrm{Ca}^{2+}$ selective CPISEs have also been constructed through the direct addition of a neutral ionophore (ETH 1001) to the soluble PANI [33] or by simply using the $\mathrm{Ca}^{2+}$-selectivity of the phosphoric acid dopants, incorporated into the membrane [34,35]. In the case of the phosphoric acid dopants either bis(2-ethylhexyl)phosphoric acid [34] or bis[4-(1,1,3,3-tetramethylbutyl)phenyl]phosphoric acid (DTMBP-PO $\left.{ }_{4} \mathrm{H}\right)$ [35] were used as the protonating acid. Some of conducting polymer can be made soluble by treating them with functionalized organic acids, e.g. sulfonic acids and organophosphates [35], which make them at the same time electrically conducting and soluble. Table 1 shows the characterizations of the most reported conducting polymer based ion selective electrodes.

Table 1. The characterizations of a number of reported conducting polymer based ISEs.

\begin{tabular}{|c|c|c|c|c|c|}
\hline Ions & Conducting Polymer & $\begin{array}{c}\text { Dynamic Linear } \\
\text { Range } \\
\text { (M) } \\
\end{array}$ & $\begin{array}{c}\text { Detection } \\
\text { Limit } \\
\text { (M) }\end{array}$ & $\begin{array}{c}\text { Slope } \\
\left(\mathrm{mV} \text { decade }^{-1}\right)\end{array}$ & Ref \\
\hline $\mathrm{H}^{+}-1$ & $\begin{array}{l}\text { electrochemical polymerization of ortho- } \\
\text { methoxyaniline and ortho-methylaniline }\end{array}$ & pH 2-11 & N.M & 63.8 & 36 \\
\hline $\mathrm{H}^{+}-2$ & poly(1-aminoanthracene) film & pH 1-12 & $1.0 \times 10^{-12}$ & 52.5 & 37 \\
\hline $\mathrm{H}^{+}-3$ & $\begin{array}{l}\text { cobaltabis(dicarbollide) [3,3'-Co(1,2- } \\
\left.\left.\mathrm{C}_{2} \mathrm{~B}_{9} \mathrm{H}_{11}\right)\right]^{(-)} \text {-doped polypyrrole (PPy) }\end{array}$ & pH 3-12 & $1.0 \times 10^{-12}$ & 50 & 38 \\
\hline $\mathrm{H}^{+}-4$ & polyaniline and its substituted derivatives & pH 2-9 & $1 \times 10^{-9}$ & $62.4 \pm 0.9$ & 39 \\
\hline
\end{tabular}


Table 1. Cont.

\begin{tabular}{|c|c|c|c|c|c|}
\hline Ions & Conducting Polymer & $\begin{array}{c}\text { Dynamic Linear } \\
\text { Range (M) }\end{array}$ & $\begin{array}{l}\text { Detection } \\
\text { Limit (M) } \\
\end{array}$ & $\begin{array}{c}\text { Slope } \\
\left(\mathrm{mV} \text { decade }^{-1}\right) \\
\end{array}$ & Ref \\
\hline $\mathrm{H}^{+}-5$ & polyaniline (PANI) & pH 2-9 & $1 \times 10^{-9}$ & 52.7 & 40 \\
\hline $\mathrm{H}^{+}-6$ & $\begin{array}{l}\text { polypyrrole with the dopant anion cobalt } \\
\text { bis(dicarbollide) }\left[3,3^{\prime}-\mathrm{Co}\left(1,2-\mathrm{C}_{2} \mathrm{~B}_{9} \mathrm{H}_{11}\right)_{2}\right]^{(-)}\end{array}$ & pH 2-9 & $1 \times 10^{-9}$ & 59.8 & 41 \\
\hline $\mathrm{H}^{+}-7$ & polypyrrole (P-Py) & $10^{-6}-3$ & N.M & 45.5 & 42 \\
\hline $\mathrm{H}^{+}-8$ & polypyrrole & N.M & N.M & 58 & 43 \\
\hline $\mathrm{H}^{+}-9$ & poly(aniline) ultrathin films & $\mathrm{pH} 3-9$ & $1.0 \times 10^{-9}$ & $55-59$ & 44 \\
\hline $\mathrm{Na}^{+}$ & polypyrrole (PPy), doped with NaBF4 & $10^{-5}-10^{-1}$ & $3.0 \times 10^{-5}$ & 59.2 & 45 \\
\hline $\mathrm{K}^{+}-1$ & polypyrrole & $10^{-7}-10^{-1}$ & $10^{-7.4}$ & 65.9 & 46 \\
\hline $\mathrm{K}^{+}-2$ & poly(3-octylthiophene) and valinomycin & $10^{-5}-10^{-1}$ & $5 \times 10^{-5}$ & 49 & 47 \\
\hline $\mathrm{K}^{+}-3$ & polypyrrole & $10^{-5}-10^{-1}$ & $1.0 \times 10^{-5}$ & 53.5 & 48 \\
\hline $\mathrm{K}^{+}-4$ & polyaniline (PANI) & $10^{-6}-10^{-1}$ & N.M & 58.2 & 49 \\
\hline $\mathrm{K}^{+}-5$ & poly(3,4-ethylenedioxythiophene) & $10^{-6}-10^{-1}$ & N.M & - & 50 \\
\hline $\mathrm{K}^{+}-6$ & poly(3,4-ethylenedioxythiophene) & $10^{-5}-10^{-1}$ & $10^{-5}$ & 56.4 & 51 \\
\hline $\mathrm{K}^{+}-7$ & $\begin{array}{l}\text { hexacyanoferrate(II)/(II) doped } \\
\text { polypyrrole }\end{array}$ & $10^{-5}-10^{-1}$ & $10^{-5}$ & 24.3 & 52 \\
\hline $\mathrm{K}^{+}-8$ & $\begin{array}{l}\text { polypyrrole doped with di(2-ethylhexyl) } \\
\text { sulfosuccinate }\end{array}$ & $10^{-6}-10^{-1.5}$ & $1.0 \times 10^{-6}$ & - & 53 \\
\hline $\begin{array}{l}\mathrm{K}^{+} \text {and } \\
\mathrm{Cu}\end{array}$ & poly(3-octylthiophene) & $\begin{array}{l}10^{-6}-10^{-1} \text { and } \\
10^{-7}-10^{-1}\end{array}$ & $\begin{array}{l}5.8 \times 10^{-5} \text { and } \\
6.8 \times 10^{-5}\end{array}$ & 58 and 54 & 54 \\
\hline $\begin{array}{l}\mathrm{Mg}^{2+} \\
\text { and } \\
\mathrm{Ca}^{2+}\end{array}$ & poly(3,4-ethylenedioxythiophene) & $10^{-5}-10^{-1}$ & $1.0 \times 10^{-5}$ & 29.1 and 28.6 & 55 \\
\hline $\mathrm{Ca}^{2+}-1$ & $\begin{array}{l}\text { polyaniline functionalized with bis[4- } \\
\text { (1,1,3,3-tetramethylbutyl)phenyl]- } \\
\text { phosphate }\end{array}$ & $10^{-1}$ to $10^{-4}$ & $8 \times 10^{-7}$ & $27.8 \pm 0.2$ & 56 \\
\hline $\mathrm{Ca}^{2+}-2$ & polyaniline and di(2-ethylhexyl)phosphate & $10^{-1}-10^{-3}$ & $10^{-4}$ & $27.0 \pm 0.4$ & 57 \\
\hline $\mathrm{Ca}^{2+}-3$ & polypyrrole based & $0.1-10^{-10}$ & $10^{-5}$ & 27.3 & 58 \\
\hline $\mathrm{Ca}^{2+}-4$ & polyaniline and di(2-ethylhexyl)phosphate & $10^{-3}-10^{-1}$ & $5 \times 10^{-3}$ & $28.6 \pm 1.1$ & 59 \\
\hline $\begin{array}{l}\mathrm{Li}^{+} \\
\mathrm{Ca}^{2+} \\
\text { and } \mathrm{Cl}^{-}\end{array}$ & poly(3-octylthiophene) & $\begin{array}{l}10^{-4}-10^{-1}, \\
10^{-7}-10^{-1} \\
\text { and } \\
10^{-6}-10^{-1}\end{array}$ & $\begin{array}{l}3 \times 10^{-4} \\
6 \times 10^{-7} \text { and } \\
9 \times 10^{-6}\end{array}$ & $\begin{array}{l}56.5 \\
30 \text { and } \\
62.6\end{array}$ & 60 \\
\hline $\begin{array}{l}\mathrm{Ca}^{2+} \\
\text { and } \mathrm{K}^{+}\end{array}$ & $\begin{array}{l}\text { poly(3,4-ethylenedioxythiophene) doped } \\
\text { with poly(4-styrenesulfonate) }\end{array}$ & $\begin{array}{l}10^{-9}-10^{-1} \text { and } \\
10^{-6}-10^{-1}\end{array}$ & $\begin{array}{l}5 \times 10^{-9} \text { and } \\
4.4 \times 10^{-7}\end{array}$ & 48.8 & 61 \\
\hline $\mathrm{Sr}^{2+}$ & polyaniline/polycarbonate & $1 \times 10^{-10}$ to $1 \times 10^{-9}$ & N.M & - & 62 \\
\hline $\begin{array}{l}\mathrm{Pb}^{2+} \text { or } \\
\mathrm{Ca}^{2+}\end{array}$ & poly(3,4-ethylenedioxythiophene) & $\begin{array}{l}10^{-5}-10^{-2} \text { and } \\
10^{-4}-10^{-1}\end{array}$ & $\begin{array}{l}5.0 \times 10^{-5} \text { and } \\
5.0 \times 10^{-4}\end{array}$ & $\begin{array}{l}29.9 \text { and } \\
27.3\end{array}$ & 63 \\
\hline $\mathrm{Pb}^{2+}-1$ & polypyrrole & $10^{-8.5}-10^{-5}$ & $10^{-9}$ & 58.2 & 64 \\
\hline $\mathrm{Cu}^{2+}-1$ & $\begin{array}{l}\text { poly(3,4-ethylenedioxythiophene) films } \\
\text { doped by hexacyanoferrate anions }\end{array}$ & $10^{-6}-10^{-2}$ & $10^{-6}$ & 30 & 65 \\
\hline
\end{tabular}


Table 1. Cont.

\begin{tabular}{|c|c|c|c|c|c|}
\hline Ions & Conducting Polymer & $\begin{array}{l}\text { Dynamic Linear } \\
\text { Range (M) }\end{array}$ & $\begin{array}{l}\text { Detection } \\
\text { Limit (M) }\end{array}$ & $\begin{array}{c}\text { Slope } \\
\left(\mathbf{m V} \text { decade }^{-1}\right) \\
\end{array}$ & Ref \\
\hline $\mathrm{Cu}^{2+}-2$ & $\begin{array}{l}\text { poly(3,4-ethylenedioxythiophene) } \\
\text { (PEDOT) doped with 2-(o-arseno- } \\
\text { phenylazo)-1,8-dihydroxynaphthalene- } \\
\text { 3,6-disulphonic sodium salt (Arsenazo- } \\
\text { 1) }\end{array}$ & N.M & N.M & 59 & 66 \\
\hline $\mathrm{Ag}^{+}-1$ & $\begin{array}{l}\text { poly(3,4-ethylenedioxythiophene) } \\
\text { (PEDOT) }\end{array}$ & $10^{-5}-10^{-1}$ & $5 \times 10^{-5}$ & 56 & 67 \\
\hline $\mathrm{Ag}^{+}-2$ & poly(3-octylthiophene) (POT) & $10^{-4}-10^{-1}$ & $10^{-5.5}$ & 49 & 68 \\
\hline $\mathrm{Ag}^{+}-3$ & poly(3,4-ethylenedioxythiophene) & $10^{-4}-10^{-1}$ & $10^{-5.3}$ & 60.7 & 69 \\
\hline $\mathrm{Ag}^{+}-4$ & $\begin{array}{l}\text { poly(3,4-ethylenedioxythiophene) doped } \\
\text { with silver hexabromocarborane }\end{array}$ & $10^{-5}-10^{-1}$ & $1.0 \times 10^{-5}$ & $40.5 \pm 1.6$ & 70 \\
\hline $\mathrm{Ag}^{+}-5$ & $\begin{array}{l}\text { poly(3,4-ethylenedioxythiophene) and } \\
\text { polypyrrole doped with sulfonated } \\
\text { calixarenes }\end{array}$ & $10^{-5}-10^{-1}$ & $10^{-5.1}$ & 55.7 & 71 \\
\hline $\begin{array}{l}\mathrm{Zn}^{2+} \text { and } \\
\mathrm{K}^{+} \\
\end{array}$ & $\begin{array}{l}\text { tetraphenylborate (TPB) ion doped } \\
\text { polypyrrole }\end{array}$ & $10^{-5.6}-10^{-1}$ & $10^{-6}$ & 58 & 72 \\
\hline $\mathrm{Hg}^{2+}$ & polypyrrolel/polyantimonic acid & $10^{-5}-10^{-1}$ & $5.0 \times 10^{-5}$ & 29.6 & 73 \\
\hline $\mathrm{CO}_{3}{ }^{2-}$ & $\begin{array}{l}\text { [poly(1-hexyl-3,4-dimethyl-2,5- } \\
\text { pyrrolylene) }\end{array}$ & $1 \times 10^{-4}$ to $1 \times 10^{-1}$ & $2.6 \times 10^{-4}$ & -29.5 & 74 \\
\hline $\mathrm{NO}_{3}^{-}-1$ & doping of polypyrrole & $5.0 \times 10^{-5}-0.50$ & $2 \times 10^{-5}$ & $-56 \pm 1$ & 75 \\
\hline $\mathrm{S}^{2-}$ & $\begin{array}{l}\text { poly(3-methylthiophene) and poly- } \\
\text { (dibenzo-18-crown-6) }\end{array}$ & $5.0 \times 10^{-8}-1.0 \times 10^{-3}$ & $2.0 \times 10^{-9}$ & -35.7 & 76 \\
\hline $\begin{array}{l}\mathrm{F}^{-} \\
\mathrm{H}_{2} \mathrm{PO}_{4}^{-}\end{array}$ & polyaniline modification & $10^{-3}-10^{-1}$ & N.M & -45 and -39 & 77 \\
\hline $\mathrm{Cl}^{-}-1$ & $\begin{array}{l}\text { poly(3-octylthiophene) and } \\
\text { tridodecylmethylammonium chloride }\end{array}$ & $10^{-4}-10^{-1}$ & $5.0 \times 10^{-5}$ & -58 & 78 \\
\hline $\mathrm{Cl}^{-}-2$ & poly(3-octylthiophene) (POT) & $10^{-4}-10^{-1}$ & $5.0 \times 10^{-4}$ & -55.1 & 79 \\
\hline $\mathrm{Cl}^{-}-3$ & $\begin{array}{l}\text { poly(pyrrole) layers doped with chloride } \\
\text { (PPyCl) }\end{array}$ & $\begin{array}{l}2 \times 10^{-5}-2 \times 10^{-3} \text { and } \\
10^{-5}-2 \times 10^{-3}\end{array}$ & N.M & -58.1 and -33.1 & 80 \\
\hline $\mathrm{Cl}^{-}-4$ & polypyrrole & $10^{-7}-1$ & $4 \times 10^{-7}$ & -55.9 & 81 \\
\hline $\mathrm{Cl}^{-}-5$ & polypyrrole & $10^{-4}-1$ & $1.4 \times 10^{-4}$ & -49.8 & 82 \\
\hline $\mathrm{Cl}^{-}-6$ & $\begin{array}{l}\text { poly(3,4-ethylenedioxythiophene) } \\
\text { (PEDOT) }\end{array}$ & $10^{-4}-1$ & $7 \times 10^{-5}$ & -44.4 & 83 \\
\hline $\mathrm{Br}^{-}$ & $\begin{array}{l}\text { poly(methylthiophene-methylpyrrole) } \\
\text { copolymer }\end{array}$ & $10^{-4}-10^{-1}$ & $6 \times 10^{-5}$ & -50 & 84 \\
\hline$I^{-}-1$ & $\begin{array}{l}\text { poly(3-methylthiophene) conducting } \\
\text { polymer }\end{array}$ & $1 \times 10^{-7}-5 \times 10^{-1}$ & $1 \times 10^{-8}$ & - & 85 \\
\hline $\begin{array}{l}\text { dodecyl } \\
\text { sulfate }\end{array}$ & dodecylsulfate-doped polypyrrole & $10^{-5}-7 \times 10^{-3}$ & $5 \times 10^{-6}$ & -57.5 & 86 \\
\hline
\end{tabular}

A brief description of potentiometric sensors assimilating conducting polymers is presented. There are several reports of ion-selective sensors based on conducting polymers including about nine reports 
about $\mathrm{H}^{+}$sensors including different conducting polymers often doped with different agents [36-44]. Hutchins and colleagues reported in $1993 \mathrm{a} \mathrm{pH}$ sensor with a linear dynamic range of $10^{-11}$ to $10^{-2} \mathrm{M}$ concentration of $\mathrm{H}^{+}$. $\mathrm{A} \mathrm{Li}^{+}$assay was reported by Bobacka et al. in 1994 using a potentiometric sensor that included a conducting poly (3-octylthiophene) polymer, which was also investigated with $\mathrm{Ca}^{2+}$ and $\mathrm{Cl}^{-}$[60]. There is a single report of $\mathrm{Na}^{+}$sensor by Cadogan et al. in 1992, where the detection limit for sodium ions was reported to be $3 \times 10^{-5} \mathrm{M}$ [45]. Eight $\mathrm{K}^{+}$selective sensors were developed during 19992007, among which the best detection limit was $10^{-7.4} \mathrm{M}$, reported by Pawlowski et al. in 2006 [46-53]. In the same year Paczosa-Bator et al. obtained a slope of 29.1 for the calibration curve for a $\mathrm{Mg}$ sensitive conducting polymer doped with ATP [55]. Over a three year period (2003-2006) seven $\mathrm{Ca}^{2+}$ ion sensors based on different conducting polymers were reported [56-62]. MMA/DMA copolymer was incorporated as a conducting polymer in a $\mathrm{Ca}^{2+}$ sensitive sensor by Sutter et al. in 2004. The detection limit was determined to be $10^{-9.3} \mathrm{M}$. A sensor for strontium ions which included polymers of polyaniline with polycarbonate was fabricated in 2002 [64]. In 2006 and 2007 two $\mathrm{Cu}^{2+}$ electrochemical sensors based on a common conducting polymer were reported while the doping agents were not the same $[65,66]$. Poly(3,4-ethylenedioxythiophene) films doped by hexacyanoferrate anions was one combination used as a conducting polymer for $\mathrm{Cu}$ (II) selective sensor. Five papers on $\mathrm{Ag}^{+}$sensors based on conducting polymers were published [67-70]; three of them were in 1995, in which Vazques and coworkers made a sensor detecting $10^{-5.3} \mathrm{M}_{\text {of }} \mathrm{Ag}^{+}$. Khan et al. in 2004 developed a novel ion selective membrane electrode for assay of $\mathrm{Hg}$ (II) reaching a detection limit of $5 \times 10^{-5} \mathrm{M}$. A polypyrrole/polyantimonic acid combination was used as conducting polymer [73]. Pandey et al. in 2002 developed a polypyrrole based sensor for Zinc capable of determining $\mathrm{Zn}$ ions in the linear range of $10^{-1}$ to $10^{-5.6}$ [72]. Lead ions were determined in one case in 2007, in which Kissiel et al. reported a linear dynamic range of $10^{-2}$ to $10^{-5} \mathrm{M}$ for a calibration curve with a slope of 29.9 for $\mathrm{Pb}$ ions [63].

Some anions also have been under investigation by conducting polymer based electrochemical sensors during the last decade. The first halogen $\left(\mathrm{F}^{-}\right)$was determined together with $\mathrm{H}_{2} \mathrm{PO}_{4}$ with a modified polyaniline sensor by Shiskanove et al. in 2005 [77]. Six publications have probed chloride ion sensors during 1999 to 2004 [78-83]. The best detection limit was achieved by Michalska et al. in 2003. They obtained a detection limit of $4 \times 10^{-7} \mathrm{M}$ utilizing a polypyrrole as conducting polymer. Another halogen $\left(\mathrm{Br}^{-}\right)$was investigated once in 1997 by Wang et al. achieving a detection limit of $6 \times 10^{-5} \mathrm{M}$ for $\mathrm{Br}^{-}$[84]. In $1994 \mathrm{Galal}$ et al. reported an iodide selective sensor and there was another report on an iodide sensor in 1999 by Wolf et al. in which $\mathrm{I}^{-}$was determined down to $5 \times 10^{-4} \mathrm{M}[85,86]$. A potentiometric sulfide selective electrode based on two different conducting polymers with a fine performance was developed by Atta et al. The detection limit of their proposed electrode was $8 \times 10^{-7} \mathrm{M}$ of sulfide ions [76]. Hutchins et al. also investigated a $\mathrm{NO}_{3}{ }^{-}$selective electrode in 1995 [75]. The linear dynamic range of the latter report was $8.0 \times 10^{-5}$ to $2.5 \times 10^{-2} \mathrm{M}$ of $\mathrm{NO}_{3}^{-}$. In 2002 Song et al. reported a $\mathrm{CO}_{3}{ }^{2-}$ sensor incorporating poly(1-hexyl-3,4-dimethyl-2,5-pyrrolylene) as conducting polymer [74]. Another organic compound, dodecylsuphate (DDS), was determined by a conducting polymer based sensor in which the detection limit was investigated to be down to $5 \times 10^{-6} \mathrm{M}$ of DDS [86]. 


\section{Non-conducting Polymer PVC-Based ISEs}

The electrodes with mobile charged sites, illustrating ionophores (organic or inorganic) compounds which bind to cations and anions, are broadly used in complex biological and environmental samples [6]. Nowadays, the response mechanism of these electrodes has been extensively investigated [7], revealing that the scientific interest should be principally concentrated on the research and the design of new ionophores [3].

In this way, the fabrication highly ion-selective electrodes (ISEs) become feasible [4,5]. The recent publication of several review studies [3-7] with reference to ISEs delineates their great significance. This relatively modern field has been subject to extensive research in the period of 1999-2007 when more than 100 ISEs just employing Schiff bases as ionophore [5].

\subsection{ISE membrane components}

Each polymeric membrane ISE comprises four basic components:

1. The polymeric matrix

2. The ionophore (membrane-active recognition)

3. The membrane solvent (plasticizer)

4. Ionic additives

The ISE nature and characteristics are considerably influenced by the nature and the amount of each component. As far as the polymeric membrane is concerned, it separates the test solution from the inner compartment, containing the target ion solution [4].

\subsubsection{The polymeric matrix}

Initially, to construct the liquid ISE membrane porous materials were soaked in a solution of a water-immiscible, nonvolatile, viscous organic liquid containing the dissolved ionophore. Recently, polymers have been utilized as homogeneous membrane matrices. For the preparation of a sensing membrane, a typical composition is as follows: 33\% (w/w) PVC as the polymeric matrix, 66\% plasticizer for the matrix homogenization and $1 \%$ ionophore. Regarding the first polymeric ISE membranes, their manufacture involved valinomycin as the neutral ion carrier in silicone rubber or PVC without the addition of lipophilic ionic sites. In such polymeric membranes, the polymer could provide the required physical properties (e.g. elasticity and mechanical stability). It should be stressed that these ISEs exhibited a Nernstian response, owing to the possible ionic impurity presence in the used PVC as well as in the presence of the other membrane components. Moreover, membranes with no ionic sites did not respond to the target ion concentration, as they incorporate almost completely pure membrane ingredients [7].

Aside from PVC, other polymers can be also employed in membrane fabrication. The suitability of the polymer to be employed in a sensing membrane (when the required solubility is displayed) is defined by the glass transition temperature $\left(\mathrm{T}_{\mathrm{g}}\right)$ of the polymer $(\mathrm{Tg}$ is the temperature at which an amorphous solid, such as glass or a polymer, becomes brittle on cooling, or soft on heating). The $\mathrm{T}_{\mathrm{g}}$ 
value should be below the room temperature. As a consequence, the designed membranes are fluid enough under the ambient conditions, they allow the diffusion of the membrane components, they present reasonable ionic conductivities and they illustrate the proper mechanical properties to be handled for routine processes. If the polymers are characterized by high $T_{g}$ values (e.g. the $T_{g}$ value of the high molecular weight PVC is $80{ }^{\circ} \mathrm{C}$ ), the use of plasticizers will be necessary. On the contrary, if the polymers are characterized by low $\mathrm{T}_{\mathrm{g}}$ values, such as soft polyurethanes with a low content of crystalline units, silicone rubber, poly(vinylidene chloride) and polysiloxanes, the use of plasticizers is not obligatory. In this way, the risk of the plasticizer leaching is avoided. Nevertheless, when no plasticizer is incorporated in the membrane, the ion selectivity modification by varying the plasticizer is no longer an alternative.

Different polymer types have been examined to be applied to membrane preparation. The majority of the examinations focused on the PVC derivatives with $1.8 \%$ carboxylate groups. The respective sensing devices, based on several neutral carriers, presented similar characteristics to those of the PVC matrices. The reason is that the $\mathrm{COOH}$ groups remain principally undissociated. The aminated PVC or the related polymers are at least partly protonated upon contact with the aqueous samples, having been used to fabricate the so-called ionophore-free $\mathrm{H}^{+}$-selective liquid membrane electrodes. The neutralcarrier-based $\mathrm{Na}^{+}$-selective ISEs with a vinyl chloride-vinyl alcohol copolymer (OH-PVC) matrix illustrated reduced protein-induced asymmetry effects.

The ISE membrane biocompatibility is of vital importance with regard to the clinical applications. For the in-vitro measurements, the protein deposition on the membrane surfaces causes membrane asymmetries and instabilities [6]. Then, frequent recalibration and skilled personnel are required. In contrast, in the in-vivo measurements, attention should be paid to the components leaching as they may have inflammatory, toxic and/or thrombogenic properties. Polyurethanes can decrease the inflammatory response and display excellent adhesive properties. A way to improve their biocompatibility is to covalently bond hydrophilic poly(ethylene oxide) to the surface of the polyurethane membranes. With respect to the blood compatibility, its improvement is possible by covalently attaching heparin to the membrane surface. Photocurable polymer matrices are usually used for the development of miniaturized electrodes by standard photolithography, as applied to the microelectronics technology. Such matrices, tested for the ISE membrane suitability, are the acrylates and the methacrylates, the methacrylated siloxane resins, the epoxyacrylates, polystyrene and the acrylates of urethane oligomers. Concerning the miniaturized electrodes, it is recommended to covalently attach all the membrane components, including the ionophore. To the polymer matrix, not only charged but also uncharged ionophores have been covalently bonded, seemingly with no essential loss in the electrode performance [7].

\subsubsection{The ionophore (membrane-active recognition)}

The ionophore or the ion carrier is the most vital component in a polymeric membrane sensor in terms of selectivity and selectivity. The ionophore or the membrane-active recognition can be an ion exchanger or a neutral macrocyclic compound [3]. It has molecule-sized dimensions and it contains cavities or semi-cavity to surround the target ions. The binding between the ionophore and the target 
ion is the molecular-level phenomenon, sensed by the ISE. Therefore, the various ISE selectivities towards the other ions are regarded to derive from the difference in the binding strengths between the selected ionophore, to be used in the sensor, and the different ions. The ISE function involves the phase transfer of the aqueous ions into an organic medium of the ISE, which is typically the plasticized PVC (as it will be discussed later). Actually, the transferred ions interact with the membrane components. In case that the incorporated ionophore is a simple ion-exchanging species, (e.g. a lipophilic ionic additive), the ion transfer process from the aqueous phase into the polymeric membrane of the sensor will be controlled by the lipophilicity of the exchanged ions, such as a phase transfer equilibrium. The selectivity behavior of an ion-exchanger based ISE, where the ionophore does not have any chemical recognition abilities, certainly reflects the relative lipophilicities of the studied ions. In other words, higher ion lipophilicity results in a greater sensor response towards the ion. After a number of studies conducted with ion-exchanging ISEs and liquid-liquid extraction assays, a lipophilicity-dependent selectivity pattern was concluded. This pattern, however, is valid only when the response is a function of the ion lipophilicity. For the cations, the corresponding pattern is:

$$
\mathrm{Cs}^{+}>\mathrm{Ag}^{+}>\mathrm{K}^{+}>\mathrm{NH}_{4}^{+}>\mathrm{Na}^{+}>\mathrm{Li}^{+}>\mathrm{Ca}^{2+}>\mathrm{Pb}^{2+}>\mathrm{Cu}^{2+}
$$

In parallel, the respective pattern for the anions is as follows:

$$
\mathrm{ClO}_{4}^{-}>\mathrm{SCN}^{-}>\mathrm{I}^{-}>\text {salicylate }>\mathrm{NO}_{3}^{-}>\mathrm{Br}^{-}>\mathrm{NO}_{2}^{-}>\mathrm{Cl}^{-}>\mathrm{HSO}_{3}^{-}>\text {acetate }>\mathrm{SO}_{4}{ }^{2-}>\mathrm{HPO}_{4}{ }^{2-}
$$

These patterns are known as the Hofmeister series [87]. On the other hand, the incorporation of a selectively binding ionophore into the ion-sensing membrane reduces the total free energy for the transfer of the ionophore-bound ions to the organic phase in comparison with that of the simply aqueous ions. When the ionophore binds to an ion strongly, its influence on the ion phase transfer equilibrium is higher. The complex formation constant $\left(\mathrm{K}_{\mathrm{f}}\right)$ of an ionophore and one or more of the ions is sufficiently strong, a difference is expected in the observed/calculated selectivity for the ionophore-based sensor, compared with the lipophilicity series depicted above. Likewise, if the ionophore-ion complexes are stronger, a greater difference is expected in the magnitude of the selectivity coefficients versus the lipophilicity series $[4,7]$.

\subsubsection{The membrane solvent (Plasticizer)}

The additives, which increase the plasticity or fluidity of the material to which they are added, are called plasticizers. Normally, the composition of the solvent polymeric membranes, used in the ionselective devices, is about 30-33\% (w/w) PVC and 60-66\% of a membrane solvent. Such a composition exhibits optimal physical properties, ensuring relatively high mobilities for their constituents. The membrane solvent has to be physically compatible with the polymer (i.e. have plasticizer properties), so as to give a homogeneous organic phase. Additionally, it may affect the selectivity behavior. In contrast, the selectivities of the carrier-based ISEs are significantly influenced by the membrane solvent. For example, a plasticizer change from the polar $o$-NPOE or nitrobenzene (NB) to the apolar dibutyl phthalate (DBP) reduced the $\mathrm{M}^{2+}$-selectivity of the ISE with the ionophore 2,3,8,9-tetraazacyclododeca-1,3,7,9-tetraene. This influence can be attributed to the plasticizer polarity, 
which can be estimated from the interaction of the charged species with a continuum of the given dielectric constant (Born model) [7].

Nonetheless, the plasticizer selection is performed in line with its compatibility with the ionophore (solubility reasons) as well as the final ISE application. The names/abbreviations of the most common plasticizers employed in the ISE fabrication are as follows: benzyl acetate (BA) [88], bis-(1-butylpentyl)ad ipate (BBPA) [89], bis(2-ethylhexyl) adipate (DOA) [90], bis(2-ethylhexyl) phthalate (dioctyl phthalate, DOP) [91], bis(2-ethylhexyl) sebacate (BEHS) [92], bis(n-octyl)sebacate (DOS) [93], bibenzyl ether (DBE) [94], dibutyl phthalates (DBP) [95], dibutyl sebacate (DBS) [93], didecyl phthalate (DDP) [96], 2-nitrophenyl phenyl ether (o-NPPE) [98], $o$-nitrophenyl octyl ether (o-NPOE) $[99,100]$ and tri- $n$-butyl phosphate (TBP) [101].

\subsubsection{Plasticizer-Free Polymer Membrane ISEs}

PVC has been utilized as a polymer matrix for more than 30 years [7], despite the fact that there are many drawbacks related with its usage. Plasticizer leakage is one them. Diminished sensor lifetimes, unstable responses and sample perturbation are caused by the plasticizer exudation from the sensing membranes. Furthermore, the decreased plasticizer content can reduce the ionophore solubility and the ion exchanger within the membrane, leading to a considerable sensitivity and selectivity reduction. A usual disadvantage of the plasticized-PVC observed in the ion-selective field effect transistors (ISFETs) is its poor adhesion to the gate oxide surfaces, which results in a shortened lifetime of the sensing device [7].

\subsubsection{Ionic additives}

The permselectivity of the ISE membranes is a prerequisite in order to attain a theoretical response. The permselectivity ensures that no significant amount of the counter ions may enter the membrane phase. To achieve this so-called Donnan exclusion with the electrically neutral carriers, the counter ions (ionic sites) confined to the membrane must be present. Despite the fact that the neutral-carrierbased ISE membranes could function properly, even when they contain only a very small amount of ionic sites, the addition of a lipophilic ion salt is advantageous for various other reasons. The anionic interference decrease, observed in the presence of lipophilic anions (e.g. thiocyanate) is the basic reason for the addition of a tetraphenyl borate salt to the membrane of a cation-selective electrode. Simultaneously, the electrical resistance of the membrane is diminished, which is especially important in microelectrodes. The ionic additives are ion exchangers, which themselves induce a selective response if no or only an insufficient ionophore amount is present. It becomes pretty obvious that their concentration must be carefully adjusted [7].

The most important salts used as lipophilic additives are potassium tetrakis( $p$-chlorophenyl) borate (KTPCIPB) [102,103], sodium tetrakis-[3,5-bis(1,1,1,3,3,3-hexafluoro-2-methoxy-2-propyl)phenyl] borate (NaHFPB) [104], sodium tetraphenyl borate (NaTPB) [105], tetrakis(4-fluorophenyl)borate (cesibor) [106], tetrakis[3,5-bis(trifluoromethyl)phenyl]borate (TFPB) [98] as cationic additive and 
hexadecylpyridinium bromide (HDPB) [107], hexadecyltrimethylammonium bromide (HTAB) [107], trioctylmethylammonium chloride (TOMACl) [108] as anionic additive.

A variety of tetraphenyl borate derivatives were recently employed as anionic additives. Their disadvantage is that their chemical stability is limited, especially in the presence of acids, oxidants and light. The decomposition of these compounds is attributed to the $\mathrm{H}^{+}$ions attack on the phenyl substituents. The introduction of electron withdrawing substituents may increase the stability. The best available anionic additives are sodium oleic acid (OA) [95] and potassium tetrakis[ $p$-chlorophenyl]borate (KTpClPB) [103], on account of their chemical stability and lipophilicity. Ganjali et al. reported the use of the first fatty acid (e.g. oleic acid), as an appropriate lipophilic additive, for inducing permselectivity to some PVC membrane selective electrodes. Suitable cationic additives are considered to be the lipophilic tetraalkylammonium salts, like the hexadecyltrimethylammonium bromide (HTAB) [107]. The hydrophilic counter ions of these lipophilic additives are exchanged with the primary ion as soon as the ISE is conditioned in the respective solutions.

To avoid the ionic sites leakage, their covalent bonding to the polymer matrix can be of interest (e.g. in sulfonated PVC). It should be nonetheless mentioned that the polymer could display a modified selectivity behavior, owing to the direct interaction of the sulfonate group with the cations $[4,7]$.

\subsection{Non-conducting Polymer Membrane Sensors for the Hydrogen Ion and the First Main Group Cations}

The macrocyclic ligands can form selective and stable complexes with the metal ions of compatible dimensions [94] and can potentially be applied to their separation and determination [110-112]. For these reasons, continuous interest has been focused on the design and synthesis of new functionalized macrocycles for specific applications. Polymeric carrier-based ion-selective electrodes (ISEs), which are selective towards the alkali and the alkaline earth metal ions such as sodium, potassium and lithium, have been the focus of numerous studies [7].

Potentiometric detectors based on ISEs are also considered suitable for this determination, since they offer advantages such as high selectivity, sensitivity, good precision, simplicity, portability, nondestructive analysis, ability to monitor the ion activity without extensive sample preparation and low cost. Because of the importance of developing new ionophores in the construction of liquid membrane ISEs, many cyclic and acyclic macromolecules have been introduced as ionophores.

A large number of $\mathrm{H}^{+}$-ISEs, based on different ionophores, have been introduced, during the past decade. They also exhibit excellent selectivities, which are absolutely comparable with, or in some cases, are even better than those of the glass electrodes. The names of some of the ionophores that were used in these sensors are 4,5-dibromofluorescein octadecylester (ETH 7075) [113], alkyldibenzylamine [114], porphyrin [115], 1,1(-bis(11-mercaptoundecyl) ferrocene [116], tertiary amine containing different alkyl chains [117], $N, N, N, N$-tetrabenzylethanediamine [118], p-tert-butylcalix[4]arene-oxacrown-4 [119], $N, N^{\prime}$-dialkylbenzylethylenediamine [120], tridodecylamine (TDDA) [121,122], alkyldibenzyl amines [123], and tribenzylamine [124].

During the past decade, there were four reports on $\mathrm{Li}^{+}$ion selective electrodes based on NASICONtype ceramics, 16-crown-4 derivatives, and 1,10-phenanthroline derivatives [125-128], four reports on 
$\mathrm{Na}^{+}$based on calix[4]arene triesters, cetylpyridinium-nitroprusside ion pair, dibenzopyridino-18crown-6 and silacrown ether [129-132], more than 13 reports on $\mathrm{K}^{+}$based on rifamycin, benzo-15crown-5, gamma-cyclodextrin, benzo-15-crown-5 fluoroionophore, dibenzo-18-crown-6, 5dicarboxylic acid, decyl-18-crown-6, 4-acryloylamidobenzo-15-crown-5, bis(15-crown-5 ether) derived from xanthene-4,5-dicarboxylic acid, 2,2(-bis[3,4-(15-crown-5-)-2-nitrophenylcarbamoxymethyl] tetradecane, styrene/4(-vinylbenzo-24-crown-8) copolymer, valinomycin, bis(crown ether) ionophore containing two benzo-15-crown-5 moieties and cis-and trans-bis(crown ether)s [133-145], for $\mathrm{Rb}^{+}$ there was one report which is based on crown ethers incorporating anthraquinone, benzoquinone, and 1,4-dimethoxybezene [146] and 11 reports on $\mathrm{Cs}^{+}$ion selective electrodes based on upper-rim calix[4]crown, calix[4]arene dibenzocrown ether, lipophilic calix[6]arene tetraester derivatives, pyrone compound, quadruply-bridged calix[6]arenas, biscalix[4]arena-25,25',27.27'-bis(1,3-dioxypropane)bis(5,11,17,23-p-tert-tetrabutylcalix[4]arene-26,28-diol), calix[4]arene derivative [25-(3-bromoproploxy)-5,11,-17,23-tetra-kis(tert-butyl)-26,27,28-tris(1-propyloxy)calix[4]arene], poly(tetrafluoroethylene-co-ethylene-co-vinyl acetate), 1,3-alternate thiacalix[4]biscrown-6,6, Cs-12-molybdophosphate, sodium tetrakis-[3,5-bis(trifluoromethyl)phenyl] borate [147-157].

Figure 5. The statistical diagram of the reported potentiometric membrane sensors based on conducting and non-conducting polymer for alkali cations.

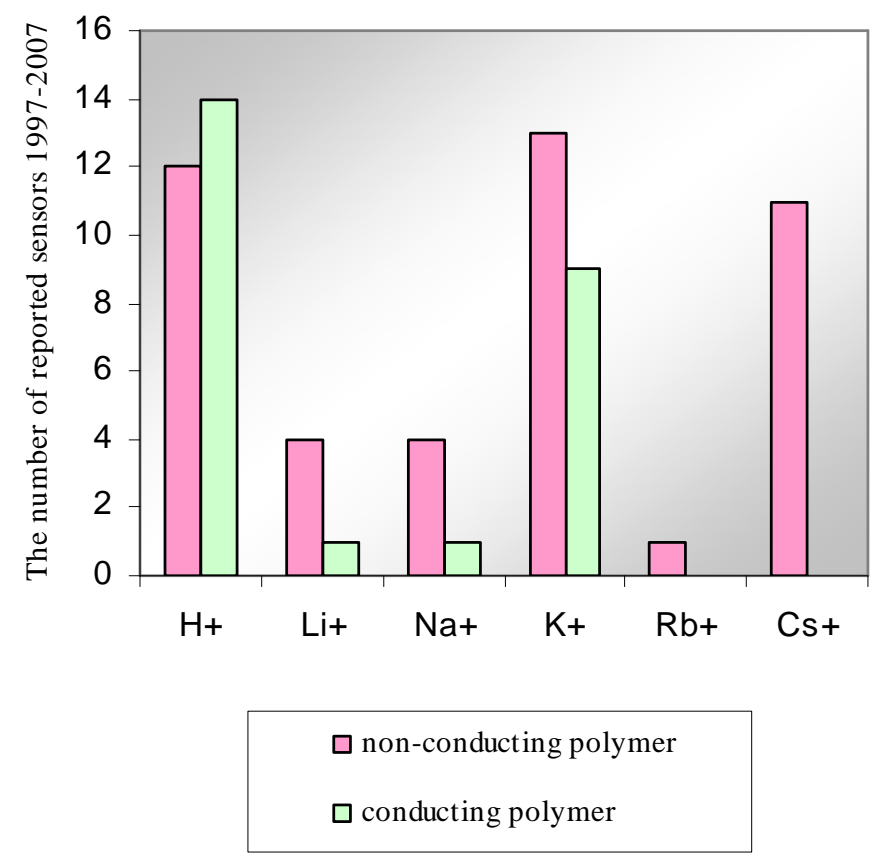

The statistical diagram of the reported potentiometric membrane sensors based on conducting and non-conducting polymer for alkali cations is shown in Figure 5.

Table 2 shows the characterization of the best reported alkali cation selective sensors based on non conducting polymer. 
Table 2. Characterization of a number of reported ion selective sensors based on nonconducting polymers for alkali cations.

\begin{tabular}{|c|c|c|c|c|c|}
\hline Cation & Ionophore & $\begin{array}{c}\text { Slope } \\
\left(\mathbf{m V} \text { decade }^{-1}\right)\end{array}$ & $\begin{array}{c}\text { Linear Range } \\
(\mathrm{M}) \\
\end{array}$ & $\begin{array}{c}\text { Most Important Interfering } \\
\text { ions }\left(\log K_{\text {sel }}>-2\right)\end{array}$ & Ref. \\
\hline $\mathrm{Li}^{+}-1$ & lipophilic crown-4 derivatives & 58 & $10^{-4}-10^{-1}$ & $\mathrm{Na}^{+}, \mathrm{K}^{+}, \mathrm{NH}_{4}^{+}$ & 125 \\
\hline $\mathrm{Li}^{+}-2$ & 1,10-Phenanthroline Derivatives & 58.7 & $10^{-4}-10^{-1}$ & $\mathrm{Na}^{+}, \mathrm{K}^{+}$ & 128 \\
\hline $\mathrm{Na}^{+}$ & 1-methyl-1-vinyl-14-crown-5 & 55.0 & $\begin{array}{l}3.16 \times 10^{-6}- \\
1.0 \times 10^{-1}\end{array}$ & $\mathrm{~K}^{+}$ & 132 \\
\hline $\mathrm{K}^{+}$ & $\begin{array}{l}\text { styrene/4(-vinyl-benzo-24-crown-8) } \\
\text { copolymer }\end{array}$ & 58 & $\begin{array}{l}1.0 \times 10^{-6}-1.0 \times \\
10^{-1}\end{array}$ & - & 140 \\
\hline $\mathrm{Rb}^{+}$ & $\begin{array}{l}\text { crown ethers incorporating } \\
\text { anthraquinone, benzoquinone, and 1,4- } \\
\text { dimethoxybezene }\end{array}$ & 54.7 & $\begin{array}{l}1.0 \times 10^{-5}- \\
1.0 \times 10^{-1}\end{array}$ & $\mathrm{Na}^{+}, \mathrm{K}^{+}, \mathrm{Mg}^{2+}, \mathrm{NH}_{4}^{+}, \mathrm{Li}^{+}$ & 145 \\
\hline $\mathrm{Cs}^{+}$ & $\begin{array}{l}\text { calix[4]arene derivative [25-(3-bromo- } \\
\text { proploxy)-5,11,-17,23-tetrakis(tert- } \\
\text { butyl)-26,27,28-tris(1- propyloxy) } \\
\text { calix[4]arene] }\end{array}$ & 58 & $\begin{array}{l}1.0 \times 10^{-6}-1.0 \times \\
10^{-1}\end{array}$ & - & 153 \\
\hline
\end{tabular}

Some of the above ionophores used in construction of non-conducting polymer ISEs for first main group cations are shown in Figure 6.

Figure 6. Structures of some ionophores used in construction of ion selective membrane sensors for some of first main group cations

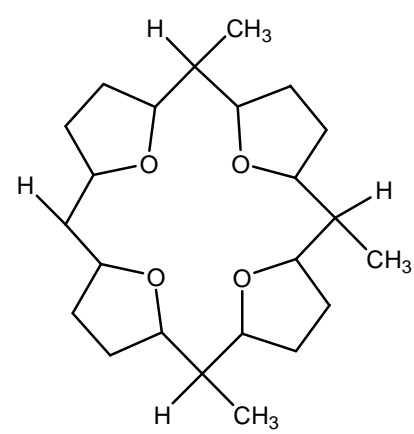

$\mathrm{Li}^{+}($Ref. 127)<smiles>c1cc2nc(c1)COc1ccccc1OCCOCCOc1ccccc1OC2</smiles>

$\mathrm{Na}^{+}$(Ref. 131)<smiles>Nc1cc(C(=O)Nc2ccc3c(c2)OCCOCCOCCOCCO3)cc(C(=O)Nc2ccc3c(c2)OCCOCCOCCOCCO3)c1</smiles>

$\mathrm{K}^{+}($Ref. 145) 


\subsection{Non-conducting Molymer Membrane Sensors for the Second Main Group Cations}

During the past decade, there were nine reports on $\mathrm{Be}^{2+}$ ion selective electrodes, mostly based on benzo-9-crown-3 and its derivatives [102, 158-165], three reports on $\mathrm{Mg}^{2+}$ based on the synthetic neutral carrier ETHT 5504, araldite zirconium(IV) selenomolybdate and benzo-15-crown-5 [166-168], more than eight reports on $\mathrm{Ca}^{2+}$ based on polyaniline functionalized with bis[4-(1,1,3,3-tetramethylbutyl)phenyl]phosphate, bilirubin (1,3,6,7-tetramethyl-4,5-dicarboxyethy-2,8-divinyl-[b13]dihydrobilenone, dibenzo-18-crown-6, [2-(2-hydroxyphenyl)imino]-1,2-diphenylethanone, ETH 1001 and ETH 129, dimethyl 1-(4-nitrobenzoyl)-8-oxo-2,8-dihydro-1H-pyrazolo[5,1-a]isoindole-2,3dicarboxylate, x-furyl dioxime [99, 169-175], For $\mathrm{Sr}^{2+}$ there were eight reports based on lipophilic diamides containing pyridine rings, dibenzo-24-crown-8 and 4-tert-butylcalix[8]arene, 5,11,17,23, 29,35-hexakis-(1,1,3,3-tetramethylbutyl)-37,38,39,40,41,42-hexakis(carboxymethoxy)-calix[6]arene, 5,7,12,14-di-benzo-2,3,9,10-tetraoxa-1,4,8,11-tetraazacyclotetradecane, 1,10-diaza-5,6-benzo-4,7dioxacyclohexa-decane-2,9-dione [174-181] and three reports on $\mathrm{Ba}^{2+}$ ion selective electrodes [182184]. The statistical diagram of the reported potentiometric membrane sensors based on conducting and non-conducting polymer for alkaline cations is shown in Figure 7.

Figure 7. The statistical diagram of the reported potentiometric membrane sensors based on conducting and non-conducting polymer for alkaline earth cations

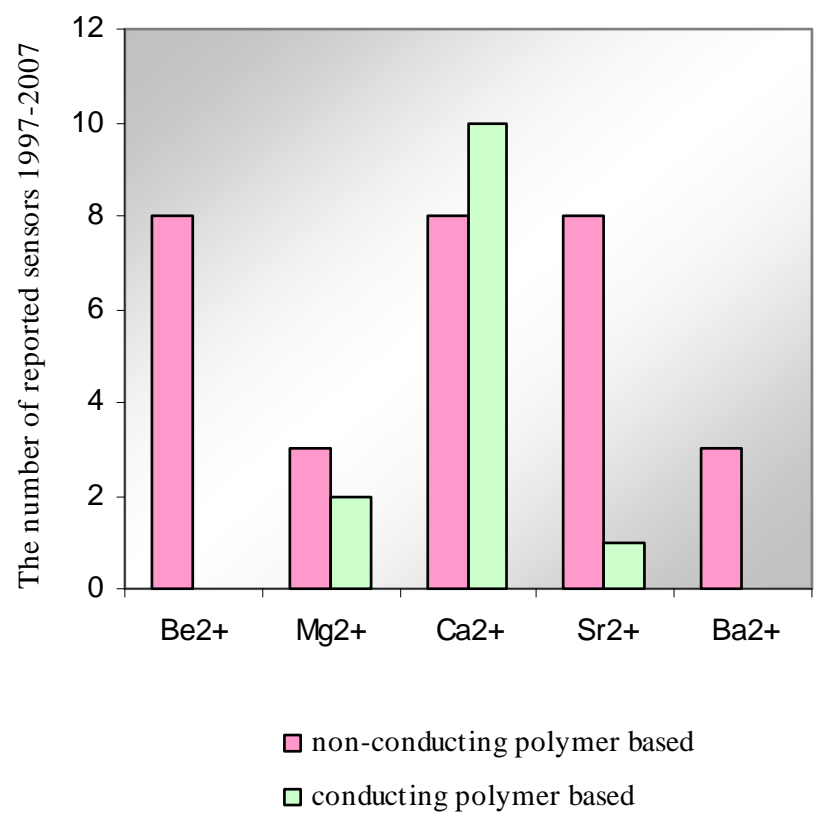

Table 3, shows the characterization and properties of the reported alkaline cation membrane sensors. 
Table 3. Characterization of a number of reported ion selective sensors based on nonconducting polymers for alkaline earth cations.

\begin{tabular}{|c|c|c|c|c|c|}
\hline Cation & Ionophore & $\begin{array}{c}\text { Slope } \\
\left(\mathbf{m V} \text { decade }^{-1}\right) \\
\end{array}$ & $\begin{array}{c}\text { Linear Range } \\
\text { (M) }\end{array}$ & $\begin{array}{c}\text { Most Important Interfering } \\
\text { ions }\left(\log K_{\text {sel }}>-2\right)\end{array}$ & Ref. \\
\hline $\mathrm{Be}^{2+}-2$ & $\begin{array}{l}\text { 2,3,5,6,8,9-hexahydro-1,4,7,10- } \\
\text { benzotetra oxacyclododecine-12- } \\
\text { carbaldehyde-12-(2,4-dinitrophenyl)hy } \\
\text { 2,6-diphenyl-4-benzo-9-crown-3- } \\
\text { pyridine }\end{array}$ & 29.9 & $\begin{array}{l}1.0 \times 10^{-7}-1.0 \times \\
10^{-1} \\
1.0 \times 10^{-7}- \\
1.0 \times 10^{-1}\end{array}$ & $\mathrm{Mg}^{+2}, \mathrm{Ca}^{+2}, \mathrm{~K}^{+}, \mathrm{Na}^{+}$ & 152 \\
\hline $\begin{array}{l}\mathrm{Mg}^{2+}-1 \\
\mathrm{Mg}^{2+}-2\end{array}$ & $\begin{array}{l}\text { synthetic neutral carrier } \\
\text { ETHT } 5504 \\
\text { araldite zirconium(IV) } \\
\text { selenomolybdate }\end{array}$ & $\begin{array}{l}28.6 \\
23\end{array}$ & $\begin{array}{l}1.0 \times 10^{-5}-1.0 \\
\times 10^{-1} \\
1.0 \times 10^{-5}-1.0 \\
\times 10^{-1}\end{array}$ & $\begin{array}{l}\mathrm{Ca}^{2+} \\
\mathrm{Ca}^{2+}\end{array}$ & 167 \\
\hline $\begin{array}{l}\mathrm{Ca}^{+2}-1 \\
\mathrm{Ca}^{+2}-2\end{array}$ & $\begin{array}{l}\text { [2-(2-hydroxyphenyl)imino]-1,2- } \\
\text { diphenylethanone } \\
\text { dimethyl 1-(4-nitrobenzoyl)-8-oxo-2,8- } \\
\text { dihydro-1H-pyrazolo[5,1-a]isoindole- } \\
\text { 2,3-dicarboxylate }\end{array}$ & 28.5 & $\begin{array}{l}1.0 \times 10^{-6}- \\
1.0 \times 10^{-1} \\
8.0 \times 10^{-7} \\
1.0 \times 10^{-1}\end{array}$ & - & 99 \\
\hline $\begin{array}{l}\mathrm{Sr}^{+2}-1 \\
\mathrm{Sr}^{+2}-2\end{array}$ & $\begin{array}{l}\text { 5,7,12,14-dibenzo-2,3,9,10-tetraoxa- } \\
\text { 1,4,8,11-tetraazacyclotetradecane } \\
\text { 1,10-diaza-5,6-benzo-4,7- } \\
\text { dioxacyclohexadecane-2,9-dione } \\
\end{array}$ & $\begin{array}{l}29.0 \\
30.0\end{array}$ & $\begin{array}{l}3.98 \times 10^{-6}-1.0 \\
\times 10^{-1} \\
1.6 \times 10^{-6}-3.0 \\
\times 10^{-3}\end{array}$ & $\mathrm{Ca}^{2+}$ & $\begin{array}{l}179 \\
180\end{array}$ \\
\hline $\mathrm{Ba}^{2+}$ & $\begin{array}{l}\text { dimethyl 1-acetyl-8-oxo-2,8-dihydro- } \\
\text { 1H-pyrazolo[5,1-a]isoindole-2,3- } \\
\text { dicarboxylate }\end{array}$ & 29.7 & $\begin{array}{l}1.0 \times 10^{-6}-1.0 \\
\times 10^{-1}\end{array}$ & - & 184 \\
\hline
\end{tabular}

Chemical structures of some of the above ionophores used in construction of non-conducting polymer ISEs for second main group cations are shown in Figure 8.

Figure 8. Structures of suitable ionophores used in construction of ion selective membrane sensors for some of second main group cations.

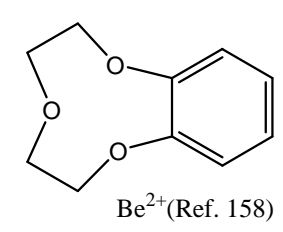<smiles>c1ccc2c(c1)OCCOCCOCCOCCO2</smiles>

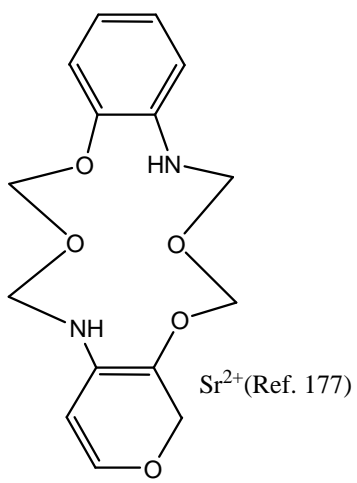<smiles>C=C/C(=C\C)C(=Nc1ccccc1O)C(=O)c1ccccc1</smiles>

$\mathrm{Ca}^{2+}($ Ref. 173) 


\subsection{Non-Conducting Polymer Membrane Sensors for the Third Main Group Cations}

The development of ISEs for trivalent cations is difficult, owing to the high hydration energy and their existence as free $\mathrm{M}^{3+}$ in narrow $\mathrm{pH}$ ranges. Due to the many industrial applications of these elements, their potentiometric monitoring is of great interest. A limited number of selective membrane sensors were developed for the cations of the IIIA Group.

During the past decade, there were five reports on $\mathrm{Al}^{3+}$ ion selective electrodes based on furyl (ethanedione, di-(2-furyl)), bis(5-phenylazosalicylaldehyde)-2,3-naphthalene diimine, hydroxythioxanthones, xanthone derivative, and $N, N$-bis(salicylidene)-1,2-phenylenediamine (salophen) [185-189], two reports on $\mathrm{Ga}^{3+}$ sensors based on clorogallium(III), 2,9-dimethyl-4,11-diphenyl-1,5,8,12-tetraazacyclotetradeca-1,4,8,11-tetraene (DDTCT) [190, 191], two reports on $\operatorname{~In~}^{3+}$ ISEs based on 1-benzyl-3methyl-4-benzoyl-5-pyrazolone, 15-crown-5-dicyclohexano-18-crown-6 [192, 193], and seven reports on $\mathrm{Tl}^{3+}$ ion selective electrodes based on quinoline-carbonitrile calix[6]arene or calix[5]arene derivatives, 1,21,23,25-tetramethyl-2,20:3,19-dimetheno-[H,2]H,23H,25H-bis-[1,3]dioxocino[5,4-i:5', 4'-i]benzo[1,2d:5.4-d']bis[1,3]benzodioxocin, 2'-amino-1,3,5'-trioxospiro[indane-2,4'(5'H)-3'-cyano indeno(1.2-b)]pyran, dibenzyldiaza-18-crown-6, tetrachlorothallate(III)-2,3,5-triphenyl-2-Htetrazolium ion pair, and $N^{\prime}$-dioctylethylenediamine- $N$ '-disuccinic acid [194-200]. The statistical diagram of the reported potentiometric membrane sensors based on non-conducting polymer for third main group cations is shown in Figure 9. According to the literature survey, there are no reports on potentiometric sensors based on conducting polymers for third main group.

Figure 9. The statistical diagram of the reported potentiometric membrane sensors based on non-conducting polymer for third main group cation.

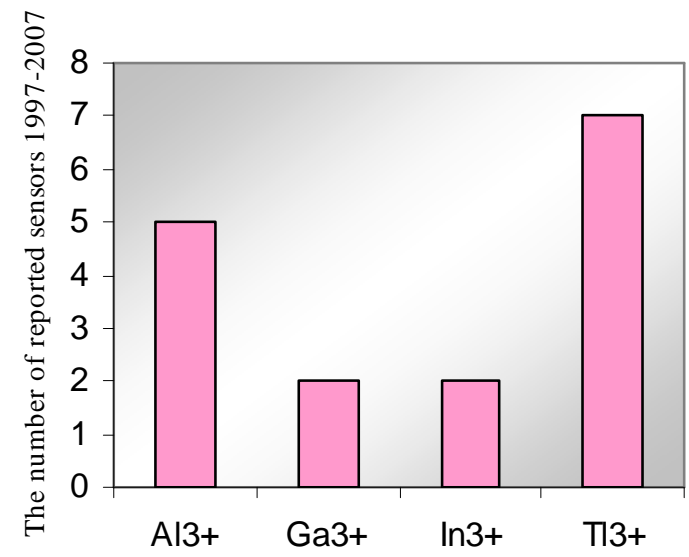

$\square$ non-conducting polymer based

Table 4, shows the characterization and properties of the reported third main group cation membrane sensors. 
Table 4. Characterization of a number of reported ion selective sensors based on nonconducting polymers for third main group cations.

\begin{tabular}{|c|c|c|c|c|c|}
\hline Cation & Ionophore & $\begin{array}{c}\text { Slope } \\
\left(\mathbf{m V} \text { decade }^{-1}\right)\end{array}$ & $\begin{array}{c}\text { Linear Range } \\
\text { (M) }\end{array}$ & $\begin{array}{c}\text { Most Important } \\
\text { Interfering ions } \\
\left(\log K_{\text {sel }}>-2\right) \\
\end{array}$ & Ref. \\
\hline $\mathrm{Al}^{+3}$ & xanthone derivative & 20.0 & $1.0 \times 10^{-6}-1.6 \times 10^{-1}$ & $\mathrm{Hg}^{2+}, \mathrm{Ba}^{2+}$ & 188 \\
\hline $\mathrm{Ga}^{3+}$ & chlorogallium(III) & 30 & $10^{-6}-10^{-2}$ & - & 190 \\
\hline $\mathrm{In}^{3+}$ & $\begin{array}{l}\text { 1-benzyl-3-methyl-4- } \\
\text { benzoyl-5-pyrazolone } \\
\text { (PMBP) }\end{array}$ & 18.7 & $3.2 \times 10^{-5}-1.0 \times 10^{-1}$ & $\mathrm{Ga}^{3+}$ & 192 \\
\hline $\mathrm{Tl}^{3+}-1$ & $\begin{array}{l}\text { 2'-amino-1,3,5'-trioxo- } \\
\text { spiro[indane-2,4'(5'H)- } \\
\text { 3'-cyano-indeno(1.2- } \\
\text { b)]pyran }\end{array}$ & 59 & $0.1-1.0 \times 10^{-6}$ & $\mathrm{Rb}^{+}, \mathrm{Cs}^{+}$ & 197 \\
\hline $\mathrm{Tl}^{3+}-2$ & $\begin{array}{l}N^{\prime} \text {-dioctylethylene- } \\
\text { diamine- } N \text { '-disuccinic } \\
\text { acid }\end{array}$ & 56 & $6.4 \times 10^{-7}-10^{-2}$ & $\mathrm{Rb}^{+}$ & 200 \\
\hline
\end{tabular}

Chemical structures of some above ionophores which are used in construction of non-conducting polymer ISEs for third main group cations are shown in Figure 10.

Figure 10. The structures of the suitable ionophores used in construction of third main group cation membrane sensors<smiles>Cc1cc(O)c2c(=O)c3ccccc3oc2c1</smiles>

$\mathrm{Al}^{3+}$ (Ref. 188)<smiles>c1ccc2c(c1)OCCOCCOCCOCCOCCOc1ccccc1O2</smiles>

$\operatorname{In}^{3+}($ Ref. 193)<smiles></smiles>

$\mathrm{Ga}^{3+}($ Ref. 190)

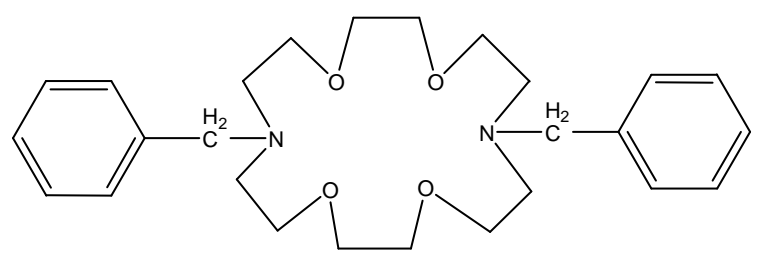

$\mathrm{Tl}^{3+}$ (Ref. 198) 


\subsection{Non-conducting Polymer Membrane Sensors for the Fourth Main Group Cations}

Tin and lead ions are the only cations of this group which have the reported membrane sensors. During the past decade, there were two reports on $\mathrm{Sn}^{2+}$ ion selective electrodes based on dibenzo-18crown-6 and 6-(4-nitrophenyl)-2,4-diphenyl-3,5-diaza-bicyclo[3.1.0] hex-2-ene [201,202], and more than 40 reports on $\mathrm{Pb}^{2+}$ ion selective electrodes based on quinaldic acid derivatives, capric acid, piroxicam, 2,2'-dithiodibenzoic acid, anthraquinone derivatives, 4-tert-butylcalix[6]arene, meso-tetrakis(2-hydroxy-1-naphthyl)porphyrin, atropisomers, dibenzodiaza-15-crown-4,4,5-diaza-9-(4-methylphenyl)imino fluorine, 1-phenyl-2-(2-quinolyl)-1,2-dioxo-2-(4-bromo) phenylhydrazone, oximinophenyl-2-ketomethyl quinoline, 1-furoyl-3-(2hydroxyethyl)thiourea, dibenzyl phosphate, tetrabenzyl pyrophosphate and diphenylphosphinic anhydride, tetraphenylporphyrin, 1-furoyl-3-phenylthiourea, 4,7,13,16-tetrathenoyl-1,10-dioxa-4,7,13,16-tetraazacyclooctadecane, 4'-vinylbenzo-15-crown-5-1,5bis[2-( $N, N$-dialkylcarbamoylmethoxy)phenoxy]3-oxa-pentanes, 1-4,1,5-bis[2-( $N, N$-dialkylcarbamoylpentadecyloxy)phenoxy]-3-oxapentanes, dithiophenediazacrown ether derivatives, $N, N^{\prime}$-bis-thiophene2-ylmethyleneethane-1,2-diamine, calix[4]arene amide derivatives, 1,8-dihydroxy-2,7-bis(prop-2'enyl)-9,10, 5,5'-dithiobis-(2-nitrobenzoic acid), bis[(1-hydroxy-9,10-anthraquinone)-2-methyl]sulfide, 1-furoyl-3,3-diethylthiourea, diporphyrin xanthene, bis(acetylacetone)-p-phenylenediamine lead(II) complex, $N, N^{\prime}$-bis(5-methylsalicylidene)- $p$-diphenylenemethane diamine, $N, N^{\prime}$-bis(3-methyl-salicylidine)-p-phenylmethane diamine, 1,10-dibenzyl-1,10-diaza-18-crown-6, $N, N^{\prime}$-bis-thiophene-2ylmethyleneethane-1,2-diamine, $N, N^{\prime}$-bis(salicylidene)-2,6-pyridinediamine Schiff's base as a neutral carrier, $N, N^{\prime}$-dibenzyl-1,4,10,13-tetraoxa-7,16-diazacyclooctadecane [203-239]. The statistical diagram of the reported potentiometric membrane sensors based on conducting and non-conducting polymer for fourth main group cations is shown in Figure 11.

Figure 11. The statistical diagram of the reported potentiometric membrane sensors based on conducting and non-conducting polymer for fourth main group cation

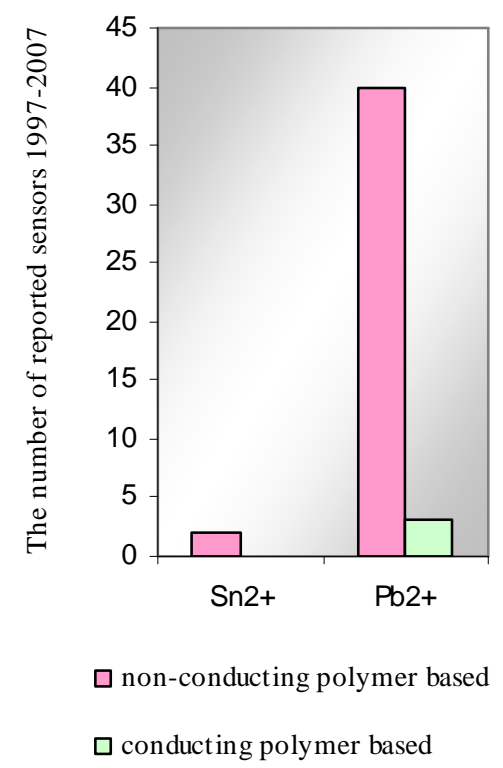

Table 5, shows the characterization and properties of the reported fourth main group cation membrane sensors. 
Table 5. Characterization of a number of reported ion selective sensors based on nonconducting polymers for fourth main group cations.

\begin{tabular}{|c|c|c|c|c|c|}
\hline Cation & Ionophore & $\begin{array}{c}\text { Slope } \\
\left(\mathbf{m V} \text { decade }^{-1}\right)\end{array}$ & $\begin{array}{c}\text { Linear Range } \\
\text { (M) }\end{array}$ & $\begin{array}{c}\text { Most Important } \\
\text { Interfering ions } \\
\left(\log K_{\text {sel }}>-2\right) \\
\end{array}$ & Ref. \\
\hline $\mathrm{Sn}^{2+}-1$ & $\begin{array}{l}\text { dibenzo-18-crown- } \\
\text { 6(DB18C6) }\end{array}$ & 27.5 & $1.0 \times 10^{-6}-1.0 \times 10^{-2}$ & - & 201 \\
\hline $\mathrm{Sn}^{2+}-2$ & $\begin{array}{l}\text { 6-(4-nitrophenyl)-2,4- } \\
\text { diphenyl-3,5-diaza- } \\
\text { bicyclo[3.1.0] hex-2- } \\
\text { ene }\end{array}$ & 28.8 & $1.0 \times 10^{-5}-1.0 \times 10^{-1}$ & - & 202 \\
\hline $\mathrm{Pb}^{2+}-1$ & $\begin{array}{l}\text { anthraquinone } \\
\text { derivative }\end{array}$ & 29.5 & $1.0 \times 10^{-7}-1.0 \times 10^{-2}$ & - & 207 \\
\hline $\mathrm{Pb}^{2+}-2$ & $\begin{array}{l}\text { 1-phenyl-2-(2- } \\
\text { quinolyl)-1,2-dioxo-2- } \\
\text { (4-bromo)phenyl- } \\
\text { hydrazone }\end{array}$ & 28.7 & $1.0 \times 10^{-6}-1 \times 10^{-1}$ & - & 222 \\
\hline $\mathrm{Pb}^{2+}-3$ & $\begin{array}{l}\text { diporphyrin } \\
\text { xanthene(ADPX) }\end{array}$ & 28.2 & $2.6 \times 10^{-6}-1.0 \times 10^{-1}$ & - & 229 \\
\hline $\mathrm{Pb}^{2+}-4$ & $\begin{array}{l}\text { N,N'-bis(salicylidene)- } \\
\text { 2,6-pyridinediamine }\end{array}$ & 29.4 & $1.0 \times 10^{-6}-1.0 \times 10^{-1}$ & $\mathrm{~K}^{+}, \mathrm{Ag}^{+}$ & 234 \\
\hline
\end{tabular}

Chemical structures of some of the above ionophores used in the construction of non-conducting polymer ISEs for fourth main group cations are shown in Figure 12.

Figure 12. Some structures of the suitable ionophores used in construction of lead and tin ion membrane sensors.<smiles>Oc1ccccc1/C=N\c1cccc(/N=C/c2ccccc2O)n1</smiles>

$\mathrm{Pb}^{2+}$ (Ref. 234)<smiles></smiles>

$\mathrm{Pb}^{2+}($ Ref. 223)<smiles>c1ccc2c(c1)OCCOCCOCCOCCO2</smiles>

$\mathrm{Sn}^{2+}$ (Ref. 201)<smiles>O=C1c2ccccc2C(=O)c2c1ccc(COCCO)c2O</smiles>

$\mathrm{Pb}^{2+}$ (Ref. 207) 


\subsection{Non-conducting Polymer Membrane Sensors for the Transition Metal Cations}

Among the 29 transition metals, liquid membrane sensors have been reported only 14 of them. Construction and application of ion selective electrode as a potentiometric sensor for determination of the ions in the real samples, offers interesting advantages such as simplicity, speed, relatively fast response, low cost, wide linear dynamic range and ease of preparation and procedures.

A literature survey reveals that during the past decade, more than 170 ion selective membrane sensors for transition metal cations have been reported. One report for $\mathrm{Y}^{3+}$ based on $S$ - $N$ Schiff's base [240], one report for $\mathrm{Zr}^{3+}$ based on bis(diphenylphosphino) ferrocene [241], one report for vanadyl ion based on $N, N^{\prime}$-bis-(salicylidene)-2,2-dimethylpropane-1,3-diamine [242], 12 reports on $\mathrm{Cr}^{3+}$ ion selective electrodes based on 2,3,8,9-tetraphenyl-1,4,7,10-tetraazacyclododeca-1,3,7,9-tetraene, a new tridentate $S, N, O$ Schiff's base 4-hydroxysalicylade-2-mercaptoanil, 2-hydroxybenzaldehyde- $O, O$ '-(1,2dioxetane-1,2-diyl) oxime, $N$-(1-thien-2-ylethylidene)benzene-1,2-diamine, 18-crown-6 (18C6), dibenzo-18-crown-6 (DB18C6) and calix[6]arene, tri-o-thymotide, oxalic acid bis(cyclohexylidene hydrazide), ion-pair $\left[\mathrm{Cr}(\text { oxalate })_{3}\right]^{3-}$ anion and tricaprylmethylammonium cation, tetraazacyclotetradecane, tetratosyltetraaza $12 \mathrm{C} 4$, tritosyltriaza 9C3, 4-dimethylaminoazobenzene, 4-amino-3hydrazino-6-methyl-1,2,4-triazin-5-one, and 1,5-diphenylcarbazide [100, 243-254], two reports on $\mathrm{Mn}^{2+}$ PVC membrane sensors based on $\left[N, N^{\prime}, N^{\prime \prime}, N^{\prime \prime \prime}-1,5,8,12\right.$-tetraazadodecane-bis(salicylaldiminato) 14,16-dimethyl-1,4,7,10,13-pentaazacyclohexadeca-13,16-diene [255,256], 11 reports on $\mathrm{Fe}^{3+}$ ion membrane sensors based on 2-[(2-hydroxy-1-propenylbuta-1,3-dienylimino)-methyl]-4-p-tolylazopheol, a mu-bis(tridentate) ligand 2-phenyl-1,3-bis[3'-aza-4'-(2'-hydroxyphenyl)-prop-4-en-1'-yl]-1,3imidazolidine, benzo-18-crown-6 crown ether, ion-pair between [Fe(oxalate) $\left.)_{3}\right]^{3-}$ anion and tricaprylylmethylammonium cation, formylsalicylic acid derivatives, 1,4,8,11-tetraazacyclotetradecane, 5,10,15,20-tetrakis(pentafluorophenyl)-21H, 23H-porphyrin, 2,4,6-tri(2-pyridyl)-1,3,5-triazine (TPTZ) [257-267].

Ten reports on $\mathrm{Co}^{2+}$ ion selective electrodes based on 5-((4-nitrophenyl)azo)- $N-\left(2^{\prime}, 4^{\prime}-\right.$ dimethoxyphenyl)salicylaldimine, $N, N^{\prime}$-bis(salicylidene)-3,4-diaminotoluene, benzo-substituted macrocyclic diamide, 2,3,4-pyridine-1,3,5,8,11,14-hexaazacyclohexadeca-2-ene, dibenzopyridino-substituted macrocyclic diamide, oxime of 1-(2-oxocyclohexyl)-1,2-cyclohexanediol, OXCCD, (2-mercapto-4methylphenyl)-2-benzamido-3-phenyl-thiopropenoate, dibenzopyridino-substituted macrocyclic diamide, 1-phenyl-3-methyl-4-benzoyl-5-benzoxypyrazole, benzo-substituted macrocyclic diamide (18-membered macrocyclic diamide) [268-279].

Fourteen reports on $\mathrm{Ni}^{2+}$ ion selective electrodes based on 5,10,15,20-tetraphenylporphyrin, 2,5thiophenyl bis(5-tert-butyl-1,3-benzoxazole), 5,11,17,23,29,35-hexakis-t-octyl-37,38,39,40,41,42hexakis( $N$-phenylthiocarbamoylmethoxy) calix[6]arene, $\quad N, N^{\prime}$-bis-(4-dimethylaminobenzylidene)benzene-1,2-diamine $\left[\mathrm{Ni}\left(\mathrm{Me}_{4} \mathrm{Bzo}_{2}[14] \mathrm{aneN}_{4}\right)\right] \mathrm{Cl}^{2-}$, benzylbis(thiosemicarbazone), 1,3,7,9,13,15, 19,21-octaazapentacyclooctacosane (pentacyclooctaaza), 3,4:11,12-dibenzo-2,5,10,13-tetraoxo1,6,9,14-tetraazacyclohexadecane, dibenzodiaza-15-crown-4, Schiff's bases, $N$-(2-hydroxybenzyl)- $N$ '(2-hydroxybenzylidene)ethylenediamine and $N$-(2-hydroxybenzylidene)-Al'-(2-picolyl)ethylenediatmine, thiophene-derivative Schiff's base, $N^{1}, N^{2}$-bis((naphthalen-1-ymethylene)ethane-1,2-diamine [280-293]. 
More than 30 reports on $\mathrm{Cu}^{2+}$ ion selective sensors based on $\mathrm{N}$-[2-thienylmethylidene]-2propanolamine, mixed complexes of $\mathrm{Cu}^{2+}$ and $\mathrm{Ni}^{2+}$ with $\mathrm{N}$-[2-thyenilmethylidene]-2-aminopyridine, naphthol-derivative Schiff's base, bis-2-thiophenal propanediamine, thiophene-derivative Schiff's base, diphenylisocyanate bis(acetylacetone) ethylenedinnine, 2,2'-[4,4'diphenyl-methanebis(nitrilomethylidyne)]-bisphenol, 2-(1'-(4'-(1"-hydroxy-2"-naphthyl)methyleneamino)butyliminomethyl)-1naphthol, derived from 2,3-diaminopyridine and omicron-vanilin 2,2-[1,2-ethandiylbis(nitrilomethylidine)-bis]metacresole(1),2,2-[1,2-ethandiyl-bis(nitrilomethylidine)-bis]-p-cresol(II) and 2,2'-[1,2-ethandiyl-bis(nitritomethylidine)-bis]-o-cresol(III), 2-\{1-(E)-2-((Z)-2-\{(E)-2-[(Z)-1-(2hydroxyphenyl)ethylidene]hydrazono)-1-methylpropylidene)hydrazono]ethyl)phenol, $N, N^{\prime}$-bis-pyridin2-ylmethylene-naphthalene-1,8-diamine, copper(II) complex of 2,4-dimethyl-1,5,9,12-tetraazacyclopentadeca-1,4-diene, 1,2,5,6,8,11-hexaazacyclododeca-7,12-dione-2,4,8,10-tetraene, Cu-II-cyclohexaneone thiosemicarbazone complex, 6,7,8,9,10-hexahydro-2H-1,13,4,7,10-benzodioxatriazacyclopentadecine-3,11(4H,12H)-dione, dithiomacrocycle (4-phenyl-11-decanoyl-1,7-dithia-11-1-azacyclotetradecane-4-sulfide), 2-(1'-(4'-(1'-hydroxy-2"-naphthyl)methyleneamino)butylimino-methyl)-1naphthol, aza-thioether crowns containing a 1,10-phenanthroline sub-unit, 2,2'-[1,9-nonanediylbis(nitriloethylidyne)]-bis-(I-naphthol6-methyl-4-(1-phenylmethylidene)amino-3-thioxo-1,2,4-

triazin-5-one, bis(acetylacetone)propylenediimine, 4-amino-6-methyl-1,2,4-triazin-5-one-3-thione, 2mercaptobenzoxazole, 9,10-anthraquinone derivative, synthesized macrocyclic diamide, copper(II) salicylaniline Schiff's base, naphthol-derivative Schiff's base, synthesized macrocyclic diamide, copper(II) complex of ethambutol, diphenylisocyanate bis(acetylacetone) ethylenedinnine, 2-quinolyl2-phenylglyoxal-2-oxime (phenylglyoxal-alpha-monoxime), 1,10-phenanthroline sub-unit, bis-2thiophenal propanediamine, 2,2'-dithiodianiline and dibutyl phthalate, cephaloridine [294-332].

Thirty nine reports on silver selective electrodes based on Schiff's base-p-tert-butylcalix[4]arene, a dioxime-type Schiff's base, $N, N^{\prime}$-bis(2'-hydroxyimino-1'-phenyl-propylidene)-1,3-propanediamine, $\mathrm{PHO}_{3}$, derived from alpha-isonitrosopropiophenone and 1,3-diaminopropane, calix[4] arene derivative, Schiff base p-tert-butylcalix[4]arene derivatives containing $\mathrm{N}$ and $\mathrm{O}$ as binding sites, [bis 5-(4nitrophenyl azo)salisylaldimine] 1,8-diamino, 3,6-dioxooctane, 2,3-butanedione-thiosemicarbazide, bis(dialkyldithiocarbamates), 2-aminothiophenol based dipodal, calix[4]arene compound of 5,11,17,23-tetra-tert-butyl-25,27-dihydroxy-calix[4]arene-thiacrown-4, calix[4]arenes in the partial cone conformation, 7,8:16,17-dibenzo-6,9,15,18-tetraoxo-1,5,10,14-tetrathiacyclooctadeca-7,16- diene $\left[\mathrm{Bz}_{2} \mathrm{Oxo}_{4} \mathrm{C}_{18}\right.$ diene $\left.\mathrm{S}_{4}\right]$ tetrathia macrocyclic carrier, calix[4]arene derivatives with four imine units, silver ethylmercurythiosalicylate (silver thimerosal), diaza-18-crown-6, containing two oxime donor groups, hexathia-18-crown-6, diphenyl selenide, benzyl phenyl selenide and dibenzyl selenide, 2,2'dithiobis(benzothiazole), meso-tetraphenylporphine $\left[\mathrm{H}_{2} \mathrm{~T}\left(4-\mathrm{OCH}_{3}\right) \mathrm{PP}\right], \quad 3-(2$-pyridylethylimino)-2butanoneoxime, 25,27-dihydroxy-26,28-bis[5-(4-methyl-6-hydroxypurimidine)thiaamyloxy] calix[4]arene, $N, N^{\prime}$-bis(2-thienylmethylene)-1,2-diaminobenzene, methyl-2-pyridyl ketone oxime (MPKO), phenyl-2-pyridyl ketone oxime and bis[2-(o-carboxythiophenoxy)methyl]-4-bromo-1-methoxybenzene, octahydroxycalix[4]arene derivative, macrocycle, Me-6(14) diene-2 $\mathrm{HClO}_{4}$, aza-thioether crowns containing a 1,10-phenanthroline sub-unit 2-mercaptobenzimidazole, octaacetyl-resorc[4]arene, calix[2]furano[2]pyrrole, exocyclic sulfur and selenium ligands based on calix[4]arenes and crown ethers, $O, O, O$-tris(2-ethylhexyl) phosphorothioate and $O, O, O$-tributyl phosphorothioate with a thiophosphoryl 
$(\mathrm{P}=\mathrm{S})$ group, cyclam (1,4,8,11-tetraazacyclotetradecane) nitrogen containing calixarene derivatives, bis-pyridine tetramide macrocycle, calixarene derivative containing nitrogen atom, bis(dialkyldithiophosphates) [333-372].

Ten reports on $\mathrm{Zn}^{2+}$ selective sensors based on $N, N^{\prime}$-bis(acetylacetone)ethylenediimine, 5,6-benzo4,7,13,16,21,24-hexaoxa-1,10-diazabicyclo[8,8,8]hexacos-5-ene, C2(B)22 cryptand, benzo-substituted macrocyclic diamide, dibenzo-24-crown-8, bis(2-nitrophenyl)disulfide, hematoporphyrin IX, sulipride drug $N$-[(ethyl-1 pyrrolidinyl-2)methyl]methoxy-2 sulfamoyl-5 benzamide, 5,6,14,15-dibenzo-1,4dioxa-8,12-diazacyclopentadecane-5,14-diene [373-382].

Fifteen reports on $\mathrm{Cd}^{2+}$ PVC membrane sensors based on incorporating nitrogen and sulfur containing tridentate dicyclohexano-24-crown-8, [1,1'-bicyclohexyl]-1,1',2,2'-tetrol, 1-furoyl-3-benzyl3-phenylthiourea, dibenzo-24-crown-8, 5-[((4-methylphenyl)azo)- $N$-(6-amino-2-pyridinyl)salicylaldimine], 5-[((4-methylphenyl)azo)- $N$-(2-diamino-2-cyano-1-ethyl cyanide) salicylaldehyde], cetylpyridiniumtetraiodo cadmate or cetylpyridinium-tetrabromo cadmate, dioctyl phthalate and sodium tetraphenyl borate, 3,4:11,12-dibenzo-1,6,9,14-tetraazacyclohexadecane, dicyclohexano-18crown-6, tetrathia-12-crown-4, 8-hydroxyquinoline, 8-hydroxyquinoline, monoaza-18-crown-6, $N, N^{\prime}$ [bis-(pyridin-2-yl)formylidene] butane-1,4-diamine and $N$-(2-pyridinylmethylene)-1,2-benzenediamine [383-395].

Twenty five reports on $\mathrm{Hg}^{2+}$ ion selective sensors based on tetraethylthiuram disulfide were chosen as a chemical modifier, bis[5-((4-nitrophenyl)azo salicylaldehyde)], p-tert-butylcalix[4]crown with imine units, sulfur Schiff's base 1-(2-hydroxy-1,2-diphenylethylidene)thiosemicarbazide, ethylenediamine bisthiophenecarboxaldehyde, ethyl-2-(benzoylamino)-3-(2-hydroxy-4-methoxyphenyl)-2-propenoate, bis(2-hydroxybenzophenone) butane-2,3-dihydrazone, dithiosalicylic acid, 4-(4$N, N$-dimethylphenyl)-2,6-diphenylpyrilium tetrafluoroborate, 2-amino-6-purinethiol and 5-amino-1,3, 4-thiadiazole-2-thiol, polyaniline Sn(IV) phosphate, 5,5'-dithio-bis(2-nitrobenzoic acid) and tricyclazole, $N, N$-dimethylformamide-salicylacylhydrazone, tribromomercurate-rhodamine B ion-pair complex, diamine donor ligand, pentathia-15-crown-5, 1-(2-nitro-4-methylphenyl)-6-methyl-6methoxy-1,4,5,6-tetrahydro-pyrimidine-2-(3H)thione, dibenzodiazathia-18-crown-6-dione, 25,27dihydroxy-26,28- bis[(1-naphthalene) selenopropoxy] calix[4]arene, 2,3,4,9,10,1,1-dipyridine-3,10diaza-1,5,8,12-tetrathiacyclotetradeca-2,9-diene, calixarene derivative containing a thiazole azo group, salicylaldehyde thiosemicarbazone, ethyl-2-benzoyl-2-phenylcarbamoyl acetate, 1,3-diphenylthiourea hexathia-18-crown-6-tetraone [396-420]. The statistical diagram of the reported potentiometric membrane sensors based on conducting and non-conducting polymer for transition metal cations is shown in Figure 13. 
Figure 13. The statistical diagram of the reported potentiometric membrane sensors based on conducting and non-conducting polymer for transition metal cation.

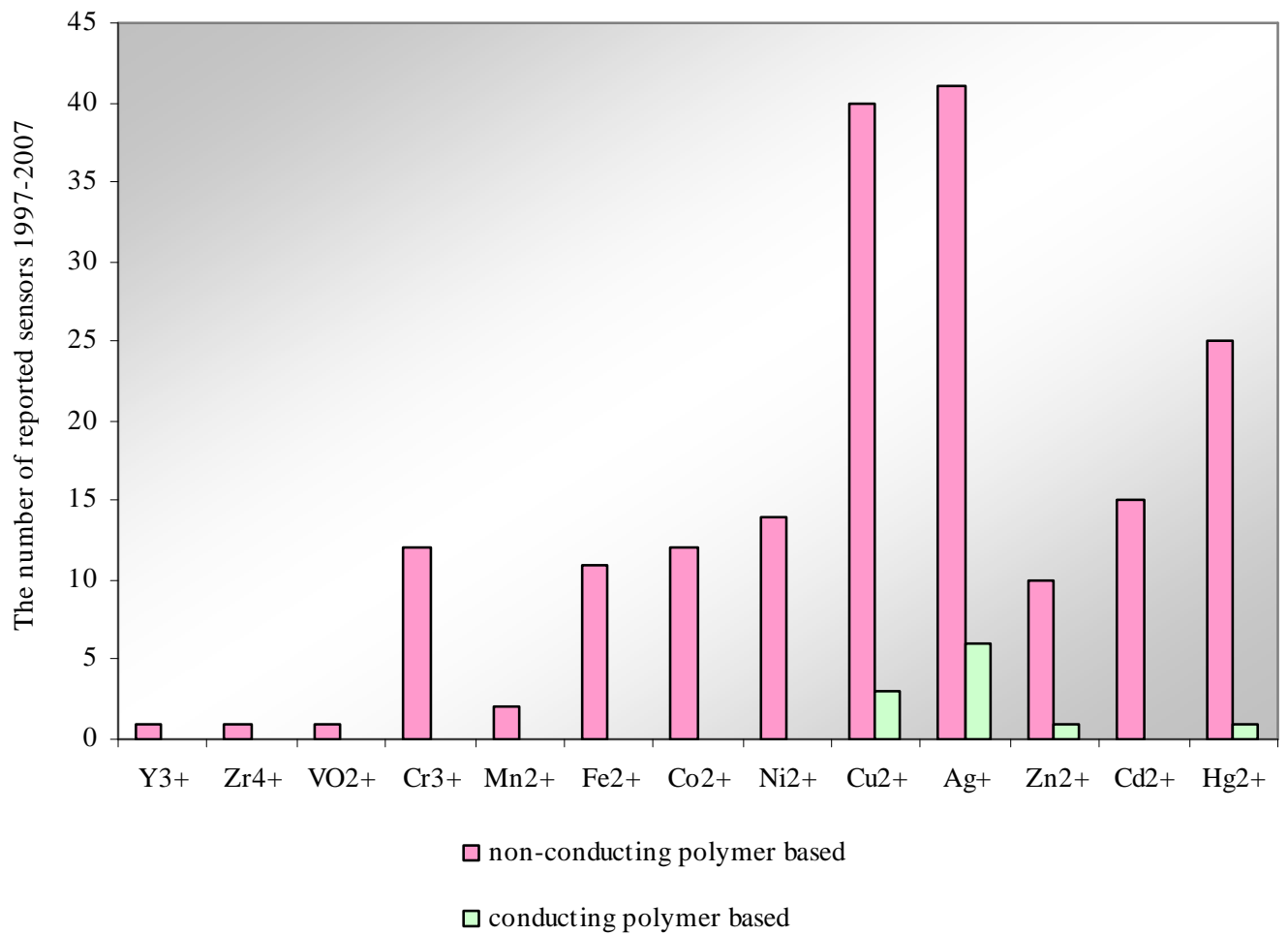

Table 6 shows the characterization and properties of the reported transition metal cation membrane sensors.

Table 6. Characterization of a number of reported ion selective sensors based on nonconducting polymers for transition metal cations

\begin{tabular}{|c|c|c|c|c|c|}
\hline Cation & Ionophore & $\begin{array}{l}\text { Slope } \\
\left(\mathrm{mV} \text { decade }^{-1}\right)\end{array}$ & $\begin{array}{l}\text { Linear Range } \\
\text { (M) }\end{array}$ & $\begin{array}{l}\text { Most Important Interfering } \\
\text { ions (log Ksel > -2) }\end{array}$ & Ref. \\
\hline $\mathrm{Y}^{3+}$ & $\begin{array}{l}\text { A new Schiff's base with sulfur and } \\
\text { nitrogen donor atoms (2- }(\{(E) 1,2 \text {-diphenyl- } \\
\text { 2-[(2-2-sulfanylphenyl)imino]ethylidene }\} \\
\text { amino)-1-benzenethiol, DSAB })\end{array}$ & 19.2 & $1.0 \times 10^{-7}-1.0 \times 10^{-2}$ & $\mathrm{Sc}^{3+}$ & 240 \\
\hline $\mathrm{Zr}^{4+}$ & bis(diphenylphosphino) ferrocene & 59.7 & $1.0 \times 10^{-7}-1.0 \times 10^{-1}$ & - & 241 \\
\hline $\mathrm{Vo}^{2+}$ & $\begin{array}{l}N, N^{\prime} \text {-bis-(salicylidene)-2,2- } \\
\text { dimethylpropane-1,3-diamine (NNPD) }\end{array}$ & & & - & 242 \\
\hline $\mathrm{Cr}^{3+}-1$ & $\begin{array}{l}\text { tetraazacyclotetradecane, tetratosyltetraaza } \\
12 \mathrm{C} 4 \text {, and tritosyltriaza } 9 \mathrm{C} 3\end{array}$ & $20 \pm 1$ & $1.0 \times 10^{-7}-1.0 \times 10^{-1}$ & $\mathrm{Fe}^{3+}, \mathrm{Ni}^{2+}$ & 250 \\
\hline $\mathrm{Cr}^{3+}-2$ & 1,5-diphenylcarbazide & $19.52 \pm 0.40$ & $6.3 \times 10^{-8}-1.0 \times 10^{-2}$ & $\mathrm{Ag}^{+}$ & 253 \\
\hline $\mathrm{Mn}^{2+}-1$ & $\begin{array}{l}N, N^{\prime}, N^{\prime \prime}, N^{\prime \prime \prime}-1,5,8,12 \text {-tetraazadodecane- } \\
\text { bis(salicylaldiminato })\left(\mathrm{H}_{2} \mathrm{~L}\right)\end{array}$ & 30 & $5.0 \times 10^{-6}-1.0 \times 10^{-1}$ & $\mathrm{Cd}^{2+}, \mathrm{Fe}^{3+}, \mathrm{Ni}^{2+}$ & 255 \\
\hline $\mathrm{Mn}^{2+}-2$ & $\begin{array}{l}\text { 14,16-dimethyl-1,4,7,10,13- } \\
\text { pentaazacyclohexadeca-13,16-diene }\end{array}$ & 29.5 & $\begin{array}{l}1.25 \times 10^{-5}- \\
1.0 \times 10^{-1}\end{array}$ & $\mathrm{Zn}^{2+,} \mathrm{La}^{3+}, \mathrm{Hg}^{2+}$ & 256 \\
\hline
\end{tabular}


Table 6. Cont.

\begin{tabular}{|c|c|c|c|c|c|}
\hline Cation & Ionophore & $\begin{array}{l}\text { Slope } \\
\left(\mathrm{mV} \text { decade }^{-1}\right)\end{array}$ & $\begin{array}{l}\text { Linear Range } \\
\text { (M) }\end{array}$ & $\begin{array}{l}\text { Most Important Interfering } \\
\text { ions (log Ksel > -2) }\end{array}$ & Ref. \\
\hline $\mathrm{Fe}^{3+}-1$ & benzo-18-crown- 6 crown ether & $15.7 \pm 1$ & $1 \times 10^{-6}-1.0 \times 10^{-1}$ & - & 259 \\
\hline $\mathrm{Fe}^{3+}-2$ & 2,4,6-tri(2-pyridyl)-1,3,5-triazine (TPTZ) & $30 \pm 1$ & $5 \times 10^{-7}-1 \times 10^{-2}$ & $\mathrm{~K}^{+}$ & 266 \\
\hline $\begin{array}{l}\mathrm{Co}^{2+}-1 \\
\mathrm{Co}^{2+}-2\end{array}$ & $\begin{array}{l}N, N^{\prime} \text {-bis(salicylidene)-3,4-diaminotoluene } \\
\text { dibenzopyridino-substituted macrocyclic } \\
\text { diamide }\end{array}$ & $\begin{array}{l}30 \pm 0.2 \\
27.5\end{array}$ & $\begin{array}{l}7.9 \times 10^{-8}-1.0 \times 10^{-1} \\
7.0 \times 10^{-7}-1.0 \times 10^{-2}\end{array}$ & $\mathrm{Cu}^{2+}, \mathrm{Ni}^{2+}, \mathrm{Cd}^{2+}$ & $\begin{array}{l}269 \\
272\end{array}$ \\
\hline $\mathrm{Ni}^{2+}-1$ & $\begin{array}{l}\mathrm{N}, \mathrm{N}^{\prime} \text {-bis-(4-dimethylamino-benzylidene)- } \\
\text { benzene-1,2-diamine }\end{array}$ & $30 \pm 1$ & $2.0 \times 10^{-7}-1.0 \times 10^{-2}$ & $\mathrm{Ag}^{+}, \mathrm{Hg}^{2+}$ & 285 \\
\hline $\mathrm{Ni}^{2+}-2$ & benzylbis(thiosemicarbazone) & $29.0 \pm 0.5$ & $1.0 \times 10^{-7}-1.0 \times 10^{-2}$ & - & 287 \\
\hline $\mathrm{Ni}^{2+}-3$ & $\begin{array}{l}1,3,7,9,13,15,19,21- \\
\text { octaazapentacyclooctacosane } \\
\text { (pentacyclooctaaza) }\end{array}$ & 30.0 & $1 \times 10^{-6}-1 \times 10^{-1}$ & $\mathrm{Ba}^{2+}$ & 288 \\
\hline $\mathrm{Ni}^{2+}-4$ & dibenzodiaza-15-crown-4 & 28.6 & $7.1 \times 10^{-7}-1.2 \times 10^{-2}$ & $\mathrm{Ag}^{+}, \mathrm{Pd}^{2+}$ & 290 \\
\hline $\mathrm{Cu}^{2+}-1$ & bis-2-thiophenal propanediamine (TPDA) & 29.1 & $6.0 \times 10^{-8}-1.0 \times 10^{-1}$ & $\mathrm{Ag}^{+}$ & 298 \\
\hline $\mathrm{Cu}^{2+}-2$ & new thiophene-derivative Schiffs base & $29.3 \pm 0.7$ & $6.0 \times 10^{-8}-1.0 \times 10^{-1}$ & $\mathrm{Zn}^{2+}, \mathrm{Hg}^{2+}$ & 299 \\
\hline $\mathrm{Cu}^{2+}-3$ & $\begin{array}{l}\text { diphenylisocyanate bis(acetylacetone) } \\
\text { ethylenedinnine (DIBAE) }\end{array}$ & 29.8 & $1.0 \times 10^{-6}-1.0 \times 10^{-1}$ & - & 300 \\
\hline $\mathrm{Cu}^{2+}-4$ & $\begin{array}{l}\text { 1,2,5,6,8,11-hexaazacyclododeca-7,12- } \\
\text { dione-2,4,8,10-tetraene }\end{array}$ & $29.5 \pm 0.3$ & $2.0 \times 10^{-7}-1 \times 10^{-1}$ & - & 308 \\
\hline $\mathrm{Cu}^{2+}-5$ & $\begin{array}{l}\mathrm{Cu}-\mathrm{II}-\mathrm{cyclohexaneone} \text { thiosemicarbazone } \\
\text { complex }\end{array}$ & 29.2 & $1 \times 10^{-9}-1 \times 10^{-1}$ & - & 309 \\
\hline $\mathrm{Cu}^{2+}-6$ & bis-2-thiophenal propanediamine (TPDA) & $29.1 \pm .1$ & $6.0 \times 10^{-8}-1.0 \times 10^{-1}$ & - & 328 \\
\hline $\mathrm{Ag}^{+}-1$ & $\begin{array}{l}\text { 25,27-dihydroxy-26,28-bis[5-(4-methyl-6- } \\
\text { hydroxypurimidine)thiaamyloxy] } \\
\text { calix[4]arene }\end{array}$ & 61.4 & $5 \times 10^{-8}-1 \times 10^{-1}$ & - & 352 \\
\hline $\mathrm{Ag}^{+}-2$ & $\begin{array}{l}\text { 2-mercaptobenzimidazole (MBI) and 2- } \\
\text { mercaptobenzothiazole (MBT) }\end{array}$ & 60.2 and 57.8 & $1.0 \times 10^{-6}-1.0 \times 10^{-1}$ & - & 354 \\
\hline $\mathrm{Ag}^{+}-3$ & cyclam (1,4,8,11-tetraazacyclotetradecane) & $59 \pm 2$ & $1.0 \times 10^{-7}-1.0 \times 10^{-1}$ & - & 363 \\
\hline $\begin{array}{l}\mathrm{Zn}^{2+}-1 \\
\mathrm{Zn}^{2+}-2\end{array}$ & $\begin{array}{l}\text { 5,6-benzo-4,7,13,16,21,24-hexaoxa-1,10- } \\
\text { diazabicyclo[8,8,8]hexacos-5-ene } \\
\text { benzo-substituted macrocyclic diamide }\end{array}$ & $\begin{array}{l}29.1 \pm 0.4 \\
28\end{array}$ & $\begin{array}{l}1.0 \times 10^{-6}-1.0 \times 10^{-1} \\
1.0 \times 10^{-9}-1.0 \times 10^{-5}\end{array}$ & $\mathrm{Li}^{+}, \mathrm{Na}^{+}$ & $\begin{array}{r}374 \\
376 \\
\end{array}$ \\
\hline $\begin{array}{l}\mathrm{Cd}^{2+}-1 \\
\mathrm{Cd}^{2+}-2\end{array}$ & $\begin{array}{l}\text { tetrathia-12-crown-4 } \\
\mathrm{N}, \mathrm{N}^{\prime} \text {-[bis(pyridm-2-yl)formylidene] butane- } \\
\text { 1,4-diamine and } \mathrm{N} \text {-(2-pyridinylmethylene)- } \\
\text { 1,2-benzenediamine }\end{array}$ & $\begin{array}{l}29.0 \pm 1.0 \\
29.5\end{array}$ & $\begin{array}{l}4 \times 10^{-7}-1.0 \times 10^{-1} \\
7.9 \times 10^{-8}-1.0 \times 10^{-1}\end{array}$ & $\begin{array}{l}- \\
\mathrm{Cu}^{2+}, \mathrm{NH}^{4+} \\
\mathrm{Cr}^{3+}\end{array}$ & $\begin{array}{l}383 \\
394\end{array}$ \\
\hline $\begin{array}{l}\mathrm{Hg}^{2+}-1 \\
\mathrm{Hg}^{2+}-2\end{array}$ & $\begin{array}{l}\text { ethylenediamine } \\
\text { bisthiophenecarboxaldehyde } \\
\text { diamine donor ligand }\end{array}$ & $\begin{array}{l}30.0 \pm 0.4 \\
25 \pm 0.1\end{array}$ & $\begin{array}{l}10^{-7}-10^{-2} \\
1.25 \times 10^{-7}-1.0 \times 10^{-1}\end{array}$ & $\mathrm{Ag}^{+}$ & 400 \\
\hline
\end{tabular}

Chemical structures of some above ionophores which are used in construction of non-conducting polymer ISEs for trantion metal cations are shown in Figure 14. 
Figure 14. Some structures of the suitable ionophores used in construction of transition metal cation membrane sensors.

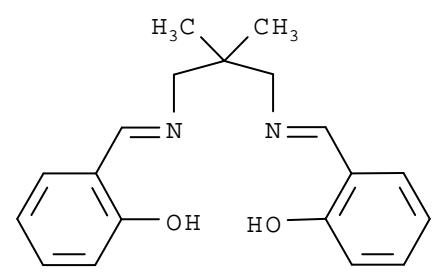

Vanadyl (Ref. 242)<smiles>c1cc2nc(c1)NCCNCCNCCNCCNCCN2</smiles>

$\mathrm{Co}^{2+}($ Ref. 271)

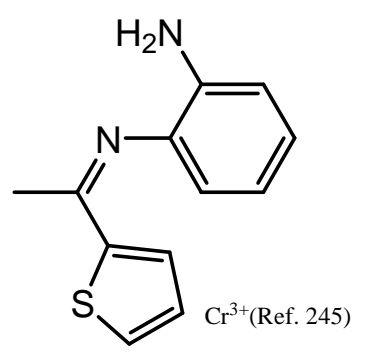<smiles>S=C(Nc1ccccc1)Nc1ccccc1</smiles>

$\mathrm{Hg}^{2+}($ Ref. 417)

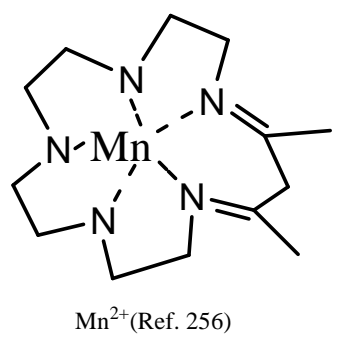<smiles>Cc1n[nH]c(=S)n(/N=C/c2ccccc2)c1=O</smiles>

$\mathrm{Cu}^{2+}($ Ref. 315)<smiles></smiles>

$\mathrm{Cd}^{2+}($ Ref. 388$)$<smiles>CN1CCCN2CCN(C)[Te]12C</smiles>

$\mathrm{Fe}^{2+}($ Ref. 264)

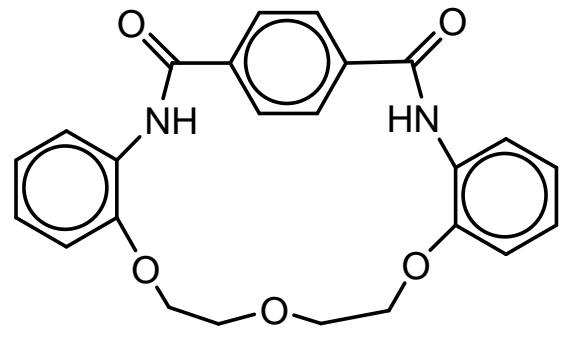

$\mathrm{Zn}^{2+}($ Ref. 376)

\subsection{Non-conducting Polymer Membrane Sensors for the Rare Earth Cations}

The main problem in the field of ISEs globally was finding a selective sensor for lanthanides. The world researchers tried to construct a selective sensor for the lanthanide ions with the aid of ionophores having cavity like crown ethers but they were not quite successful. The only way to design an ISE for the lanthanide ions is using ionophores having semi cavity, heteroatoms (mostly $\mathrm{S}$ and $\mathrm{N}$ as donor atoms), and high flexibility. Such an ionophore can easily form a template with reference to the size of the cation. Furthermore, this ionophore is able to form a stronger complex with one of the cations than with the other ones. This phenomenon can be attributed to the type, the number and the site of its donor atoms, its flexibility as well as the size and the charge density of the cation [421]. 
The literature survey reveals that during the past decade, more than 70 ion selective membrane sensors have been reported for lanthanide ions. Sixteen reports on lanthanide PVC membrane sensors based on 1,3,5-trithiacyclohexane, monoaza-12-crown-4,5,14-N,N'-hydroxyphenyl-4,15-dioxo1,5,14,18-tetraazahexacosane, $\quad N$-[hexahydrocyclopentapyrol-2((1H)yl)amino]carbonyl]-4-methylbenzene sulfonamide (gliclazide), bis(2-mercaptoanil) diacetyl, bis(thiophenol)phenylen-1,3-diamine, dicyclo-hexano-18-crown-6, N,N'-adipylbis(5-phenylazosalicylaldehyde hydrazone), bis(2-methylbenzaldehyde)butane-2,3-dihydrazone, 2,2'-dithiodipyridine, $\quad N$-2,4-dimethylphenyl- $N$ '-ethylformamidine, $N$-[hexahydrocyclopentapyrol-2((1H)yl)amino]carbonyl]-4-methyl benzene sulfonamide, 3-hydroxy- $N$ '-(pyridin-2-ylmethylene)-2-naphthohydrazide, $\quad 8$-amino- $N$-(2-hydroxybenzylidene)naphthylamine, $\quad N^{\prime}$-(1-pyridin-2-ylmethylene)-2-furohydrazide, $\quad N$-(2-pyridyl)- $N$ '-(4-methoxyphenyl)thiourea [91, 422-436].

Nine reports on cerium ion selective sensors based on 1,3,5-trithiane, 1,3,5-trithiane, azomethine of piperonylidine-4-[2.2]para-cyclophanylamine, $N$-[(Z)-2-chloro-2-(1-hydroxy-1,1,1-triphenylphosphoranyl)-1-ethenyl]-4-ethyl-1-benzene sulfonamide, 2-aminobenzothiazole and oleic acid, N,N-bis[2(salicylideneamino)ethyl]ethane-1,2-diamine, 1,4,7-trithiacyclononane and oleic acid, [4-(4'-nitrobenzyl)-1-phenyl-3,5-pyrazolidinedion)]， $\quad N^{\prime}$-[(2-hydroxyphenyl)methylidene]-2-furohydrazide [437445].

Two reports for $\operatorname{Pr}^{3+}$ membrane sensors based on $N$-(pyridin-2-ylmethylene)benzohydrazide [446,447]. Four reports for $\mathrm{Nd}^{3+}$ ion selective sensors based on $\mathrm{N}$-(2-furylmethylene) pyridine-2,6diamine, 2-\{[(6-aminopyridin-2-yl)imino]methyl $\}$ phenol, 5-pyridino-2,8-dithia[9](2,9)-1,10-phenanthrolinephane, benzyl bisthiosemicarbazone [448-451].

Six reports on $\mathrm{Sm}^{3+}$ PVC membrane sensors based on isopropyl 2-[(isopropoxycarbothioyl)disulfanyl]ethanethioate, Et 4 todit, $N$-[2-4-[[[(cyclohexylamino)carbonyl]amino]sulfonyl]phenyl]ethyl]-5-methylpyrazine carboxamide, $N$-[2-[4-[[[(cyclohexylamino)carbonyl]amino] sulfonyl]phenyl]ethyl]-5-methyl pyrazine carboxamide, 3-\{[2-oxo-1(2H)-acenaphthylenyliden]amino $\}$-2thioxo-1,3-thiazolidin-4-one, [1-phenyl-3'(2-nitrophenyl)-spiro[oxirane-2.4-pyrazoline]-3,5-dione] (PNSOP) [95, 452-455].

Four reports for $\mathrm{Eu}^{3+}$ ion selective sensors based on $N, N$-diethyl- $N$-(4-hydroxy-6-methylpyridin-2yl)guanidine, bis(thiophenol)butane2,3-dihydrazone, 4-(2-hydroxybenzylideneamino)-6-methyl-3thioxo-3,4-dihydro-1,2,4-triazi n-5(2H)-one, $S$ - $N$ hexadentate Schiff's base, bis(thiophenol)butane-2,3dihydrazone [456-460].

Four reports for $\mathrm{Gd}^{3+}$ membrane sensors based on $S-N$ Schiff's base (2-[\{3-[(2sulfanylphenyl)imino)- 1-methylbutylidene \}amino]phenyl hydrosulfide, antibiotic omeprazole, bis(thiophenal) pyridine-2,6-diamine, and $N$-(2-pyridyl)- $N$ '-(4-nitrophenyl)thiourea [105, 461-464].

Two reports on $\mathrm{Tb}^{3+}$ ion membrane sensors based on $N, N$-bis(pyrrolidene) benzene-1,2-diamine, 4amino-3-\{2-[4-amino-6-methyl-5-oxo-4,5-dihydro-1,2,4-triazin-3(2H)-yliden]hydrazono $\}$-6-methyl3,4-dihydro-1,2,4-triazin-5(2H)-one [465,466].

Four reports on $\mathrm{Dy}^{3+}$ ion membrane sensors based on $N, N$-bis(pyrrolidene) benzene-1,2-diamine, a new asymmetrical Schiff's base [(E)-N-(2-hydroxybenzylidene)benzohydraide], 6-hydrazino-1,5diphenyl-6,7-dihydropyrazolo-[3,4-d]pyrimidine-4(5H)-imine [467-470]. 
Four reports on $\mathrm{Ho}^{3+}$ PVC membrane sensors based on $N$-(1-thien-2-ylmethylene)-1,3-benzothiazol2-amine, $N, N^{\prime}$-bis(2- pyridinecarboxamide)-1,2-benzene [471-474].

Three reports on $\mathrm{Er}^{3+}$ membrane sensors based on $N^{\prime}$-(2-hydroxy-1,2-diphenylethylidene) benzohydrazide, pyridine-2-carbaldehyde-2-(4-methyl-1,3-benzothiazol-2-yl)hydrazone, and $\mathrm{N}$-(2hydroxy-1,2-diphenylethylidene) benzohydrazide [103, 475-476].

Three reports on $\mathrm{Tm}^{3+}$ ion membrane sensors for thiophene-2-carbaldehyde-(7-methyl-1,3benzothiazol-2-yl)hydrazone, 2,2'-dianiline disulfide [477-479].

Five reports on $\mathrm{Yb}^{3+}$ ion membrane sensors for 3-hydroxy- $N$-[(2-hydroxyphenyl)methylene]-2naphthohydrazide, 6-methyl-4-\{[1-(1H-pyrrol-2-yl)methylidene]amino $\}-3-t h i o x o-3,4-d i h y d r o-1,2,4-$ triazin-5(2H)-one, $N$-(6-picolyl)- $N^{\prime}$-(4-methoxyphenyl) thiourea, cefixime and $N$-(2-pyridyl)- $N^{\prime}$-(2methoxyphenyl)-thiourea [480-484].

Two reports on $\mathrm{Lu}^{3+}$ ion membrane sensors based on $\mathrm{N}$-(thien-2-ylmethylene)pyridine-2,6-diamine [485,486].

Five reports on $\mathrm{Th}^{4+}$ PVC membrane sensors based on 2-(diphenylphosphorothioyl)- $N^{\prime}, N^{\prime}$ diphenylacetamide, zirconium phosphoborate, thorium oxinate, $\mathrm{Th}\left(\mathrm{C}_{9} \mathrm{H}_{6} \mathrm{NO}\right)_{4} \cdot 2 \mathrm{H}_{2} \mathrm{O}$, thorium oxinate, $\mathrm{Th}\left(\mathrm{C}_{9} \mathrm{H}_{6} \mathrm{NO}\right)_{4} \cdot 2 \mathrm{H}_{2} \mathrm{O}, \quad 5,11,17,23$-tetra-tert-butyl-25,26,27,28-tetrakis(diphenylphosphinoylmethoxy)calix[4]arene [487-491].

However, there are no reports on lanthanide ion selective sensor based on conducting polymers. The statistical diagram of the reported potentiometric membrane sensors based on non-conducting polymer for lanthanide ions is shown in Figure 15.

Figure 15. The statistical diagram of the reported potentiometric membrane sensors based on conducting and non-conducting polymer for lanthanide ions.

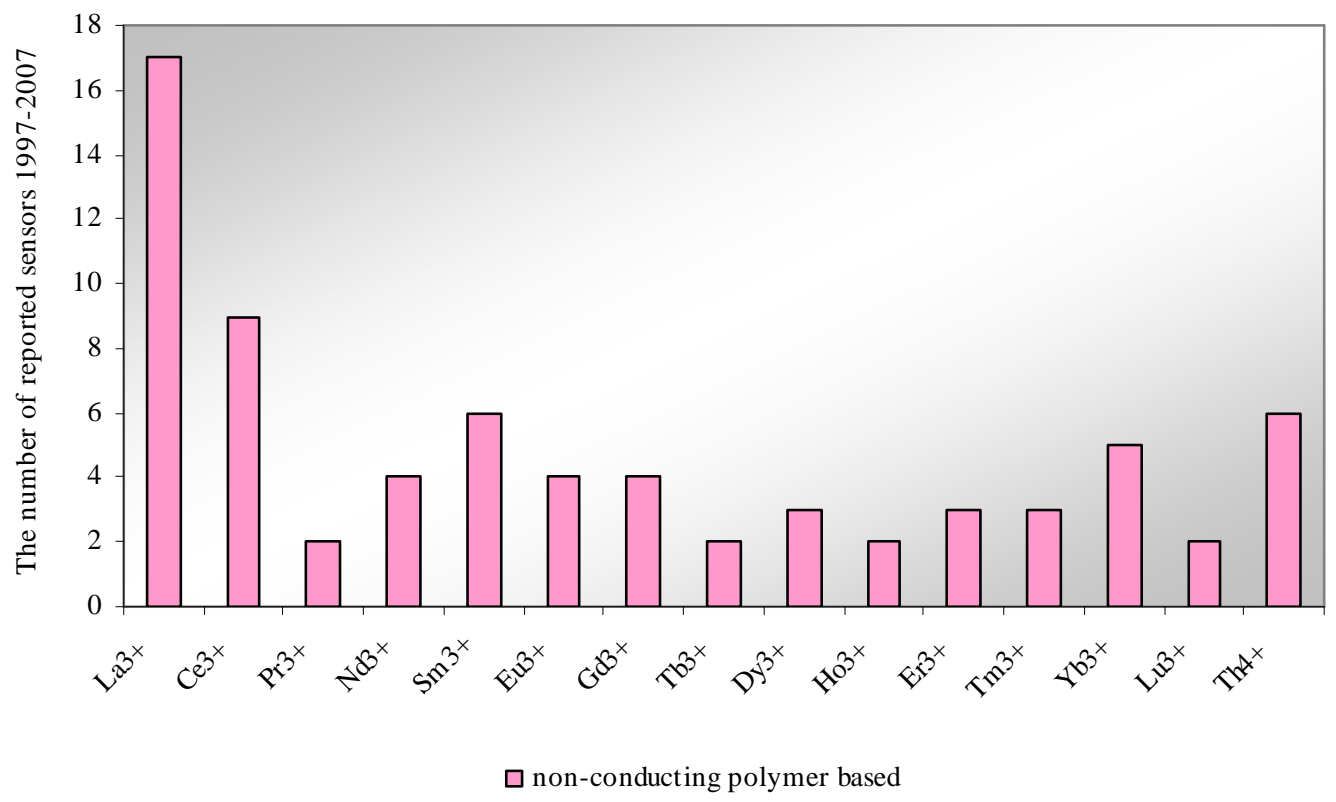

Table 7 shows the characterization and properties of the reported lanthanide ions membrane sensors. 
Table 7. Characterization of a number of reported ion selective sensors based on nonconducting polymers for lanthanide ions

\begin{tabular}{|c|c|c|c|c|c|}
\hline Cation & Ionophore & $\begin{array}{c}\text { Slope } \\
\left(\mathrm{mV} \mathrm{decade}^{-1}\right)\end{array}$ & $\begin{array}{l}\text { Linear Range } \\
\text { (M) }\end{array}$ & $\begin{array}{c}\text { Most Important } \\
\text { Interfering ions } \\
\left(\log K_{\text {sel }}>-2\right) \\
\end{array}$ & Ref. \\
\hline $\mathrm{La}^{3+}-1$ & $\begin{array}{l}N \text {-2,4-dimethylphenyl- } N^{\prime}- \\
\text { ethylformamidine (amitraz) }\end{array}$ & $19.8 \pm 0.2$ & $\begin{array}{l}1.0 \times 10^{-7}-1.0 \times \\
10^{-1}\end{array}$ & - & 432 \\
\hline $\mathrm{La}^{3+}-2$ & $\begin{array}{l}\text { 8-amino- } N \text {-(2-hydroxy- } \\
\text { benzylidene)naphthylamine }\end{array}$ & $20.3 \pm 0.3$ & $\begin{array}{l}1.0 \times 10^{-7}-1.0 \times \\
10^{-1}\end{array}$ & $\operatorname{Pr}^{3+}$ & 423 \\
\hline $\mathrm{Ce}^{3+}$ & $\begin{array}{l}N, N \text {-bis[2-(salicylidene- } \\
\text { amino)ethyl]ethane-1,2-diamine }\end{array}$ & 20 & $\begin{array}{l}1.41 \times 10^{-7}-1.0 \\
\times 10^{-2}\end{array}$ & $\mathrm{La}^{3+}$ & 442 \\
\hline $\operatorname{Pr}^{+3}$ & $\begin{array}{l}N \text {-(pyridin-2-yl-methylene)- } \\
\text { benzohydrazide }\end{array}$ & 21.1 & $10^{-2}-10^{-6}$ & $\mathrm{Sm}^{3+}, \mathrm{Er}^{3+}$ & 446 \\
\hline $\mathrm{Nd}^{+3}-1$ & $\begin{array}{l}\text { 5-pyridino-2,8-dithia[9](2,9)- } \\
\text { 1,10-phenanthrolinephane }\end{array}$ & 20.1 & $10^{-6}-10^{-2}$ & $\mathrm{Yb}^{3+}, \mathrm{Gd}^{3+}$ & 449 \\
\hline $\mathrm{Nd}^{+3}-2$ & $\begin{array}{l}\text { benzyl bisthiosemicarbazone } \\
\text { (BTC) }\end{array}$ & 19.7 & $10^{-6}-10^{-2}$ & $\mathrm{Gd}^{3+}, \mathrm{Sm}^{3+}$ & 450 \\
\hline $\mathrm{Sm}^{3+}-1$ & $\begin{array}{l}\text { 3-\{[2-oxo-1 }(2 H) \text {-acenaphthyl- } \\
\text { enyliden]amino }\} \text {-2-thioxo-1,3- } \\
\text { thiazolidin-4-one }\end{array}$ & 19.3 & $10^{-6}-10^{-1}$ & - & 455 \\
\hline $\mathrm{Sm}^{3+}-2$ & $\begin{array}{l}\text { [1-phenyl-3'(2-nitrophenyl) } \\
\text { spiro[oxirane-2.4-pyrazoline]- } \\
\text { 3,5-dione] (PNSOP) }\end{array}$ & 19.30 & $10^{-6}-10^{-1}$ & $\mathrm{Gd}^{3+}$ & 456 \\
\hline $\mathrm{Eu}^{3+}$ & $\begin{array}{l}\text { 4-(2-hydroxybenzylideneamino)- } \\
\text { 6-methyl-3-thioxo-3,4-dihydro- } \\
\text { 1,2,4-triazi n-5(2H)-one } \\
\text { (HMTDT) }\end{array}$ & $19.7 \pm 0.4$ & $\begin{array}{l}1.0 \times 10^{-6}-1.0 \\
\times 10^{-1}\end{array}$ & - & 457 \\
\hline $\mathrm{Gd}^{+3}$ & $\begin{array}{l}N \text {-(2-pyridyl)- } N \text { '-(4-nitrophenyl)- } \\
\text { thiourea }\end{array}$ & $19.95 \pm 0.3$ & $\begin{array}{l}3.0 \times 10^{-7}-1.0 \times \\
10^{-1}\end{array}$ & - & 463 \\
\hline $\mathrm{Tb}^{+3}$ & $\begin{array}{l}\text { 4-amino-3- }\{2-[4 \text {-amino-6- } \\
\text { methyl-5-oxo-4,5-dihydro-1,2,4- } \\
\text { triazin-3(2H)-ylidene] } \\
\text { hydrazono }\}-6 \text {-methyl-3,4- } \\
\text { dihydro-1,2,4-triazin-5(2H)-one } \\
\text { (ATO) }\end{array}$ & $19.4 \pm 0.5$ & $\begin{array}{l}1.0 \times 10^{-6}-1.0 \\
\times 10^{-1}\end{array}$ & - & 465 \\
\hline $\mathrm{Dy}^{+3}$ & $\begin{array}{l}\text { 6-hydrazino-1,5-diphenyl-6,7- } \\
\text { dihydropyrazolo-[3,4-d]- } \\
\text { pyrimidine-4(5H)-imine }\end{array}$ & $19.6 \pm 0.3$ & $\begin{array}{l}1.0 \times 10^{-1}- \\
1.0 \times 10^{-7}\end{array}$ & 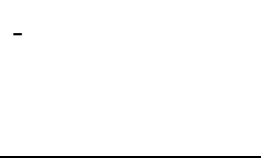 & 469 \\
\hline $\mathrm{Ho}^{+3}$ & $\begin{array}{l}N, N^{\prime} \text {-Bis(2-pyridine- } \\
\text { carboxamide)-1,2-benzene }\end{array}$ & 19.6 & $10^{-5}-10^{-2}$ & $\mathrm{Er}^{3+}, \mathrm{Dy}^{3+}, \mathrm{Sm}^{3+}$ & 472 \\
\hline $\mathrm{Er}^{+3}$ & $\begin{array}{l}N^{\prime} \text {-(2-hydroxy-1,2-diphenyl- } \\
\text { ethylidene) benzohydrazide }\end{array}$ & 21 & $10^{-7}-10^{-2}$ & - & 475 \\
\hline $\mathrm{Tm}^{+3}$ & 2,2'-dianiline disulfide (DADS) & $19.5 \pm 0.3$ & $\begin{array}{l}1.0 \times 10^{-6}-1.0 \times \\
10^{-2}\end{array}$ & - & 478 \\
\hline
\end{tabular}


Table 7. Cont

\begin{tabular}{|c|c|c|c|c|c|}
\hline Cation & Ionophore & $\begin{array}{c}\text { Slope } \\
\left(\mathbf{m V} \text { decade }^{-1}\right)\end{array}$ & $\begin{array}{l}\text { Linear Range } \\
\text { (M) }\end{array}$ & $\begin{array}{c}\text { Most Important } \\
\text { Interfering ions } \\
\left(\log K_{\text {sel }}>-2\right) \\
\end{array}$ & Ref. \\
\hline $\mathrm{Yb}^{+3}$ & $\begin{array}{l}\text { 3-hydroxy- } N \text {-[(2- } \\
\text { hydroxyphenyl)-methylene]-2- } \\
\text { naphthohydrazide }\end{array}$ & 19.2 & $10^{-7}-10^{-2}$ & $\mathrm{Nd}^{3+}, \mathrm{Pb}^{2+}, \mathrm{Gd}^{3+}$ & 480 \\
\hline $\mathrm{Lu}^{+3}$ & $\begin{array}{l}N \text {-(thien-2-ylmethylene)pyridine- } \\
\text { 2,6-diamine (TPD) }\end{array}$ & $20.5 \pm 0.4$ & $\begin{array}{l}1.0 \times 10^{-6}-1.0 \\
\times 10^{-2}\end{array}$ & $\mathrm{Nd}^{3+}, \mathrm{Dy}^{3+}, \mathrm{Gd}^{3+}$ & 485 \\
\hline $\mathrm{Th}^{4+}$ & $\begin{array}{l}\text { 2-(diphenylphosphorothioyl)- } \\
N^{\prime}, N^{\prime} \text {-diphenylacetamide }\end{array}$ & 15.2 & $10^{-6}-10^{-2}$ & $\mathrm{Mg}^{2+}, \mathrm{Cu}^{2+}$ & 487 \\
\hline
\end{tabular}

Chemical structures of some above ionophores which are used in construction of non-conducting polymer ISEs for rare earth cations are shown in Figure 16.

\subsection{Non-conducting Polymer Membrane Sensors for Inorganic Anions}

The anion selective electrodes, just like the cation selective ones, are an important group of the ion selective electrodes. The number of the anion selective electrodes is lower than that of the cationic sensors, due to reasons like the relative larger size of the anions, their various shapes and their high hydration energy. Nevertheless, a relatively large number of sensors for the anionic species have been published during the past decade.

For an anion-selective electrode, a strong interaction between the ionophore and the anion is required in order to complex the anion in a selective fashion. The potentiometric response of the membranes, doped with these complexes, is believed to be based on the coordination of the analyte anion axial ligand to the metal center of the carrier molecule.

Literature survey reveals that during the past decade more than 70 anion selective membrane sensors have been reported based on non-conducting polymers.

Three reports on bicarbonate PVC membrane sensors based on urea-functionalized calix[4]arenas, 4-(n-hexadecyl)-3-nitro-1-trifluoroacetylbenzene, and long chain $S$-alkyldiphenylthiocarbazone [492494].

Four reports on nitrite membrane sensors based on Co(II)-salen, Co(II)-salophen, (tetraphenylporphyrinato) cobalt(III) acetate, and methyl violet [495-498].

Twelve reports on nitrate ion selective sensors based on urea-calixarene, cyclic bis-thiourea, polypyrrole, silver bis(bathophenanthroline) nitrate $\left[\mathrm{Ag}\left(\right.\right.$ bath $\left.{ }_{2} \mathrm{NO}_{3}\right]$, poly(3-octylthiophene) and poly(aniline) as ion-to-electron transducers, tetradecylammonium nitrate and (npoe) 2-nitrophenyloctyl, tris(2-aminoethyl)amino triamide, tert-octylammonium bromide dissolved in dibutylphthalate, tetradecylammonium nitrate, $N, N, N$-triallylleucine betaine chloride, doped polypyrrole films, tetraoctylammonium bromide [499-510].

Three reports for dihydrogen phosphate based on cobalt rod, uranyl salophenes, and calix[4]arene anion [511-513]. 
Figure 16. Some structures of the suitable ionophores used in construction of lanthanide cation membrane sensors.

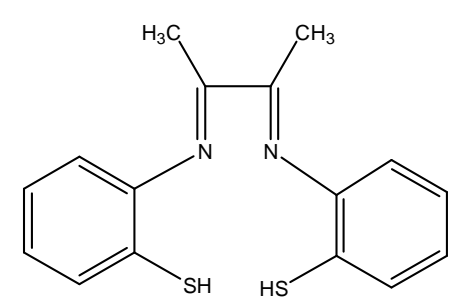

$\mathrm{La}^{3+}$ (Ref. 91)<smiles>Nc1cccc(/N=C/c2ccccc2O)n1</smiles>

$\mathrm{Nd}^{3+}$ (Ref. 449)<smiles>C(=N/c1cccc(/N=C/c2cccs2)n1)\c1cccs1</smiles>

$\mathrm{Gd}^{3+}$ (Ref. 462)<smiles>C(=N/CCC/N=C/c1ccc[nH]1)\c1ccc[nH]1</smiles>

Dy $^{3+}$ (Ref. 467)

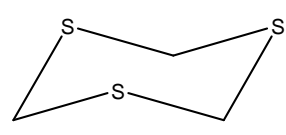

$\mathrm{Ce}^{3+}$ (Ref. 437)<smiles>O=C1C(=NN2C(=O)CSC2=S)c2cccc3cccc1c23</smiles>

$\mathrm{Sm}^{3+}$ (Ref.455)

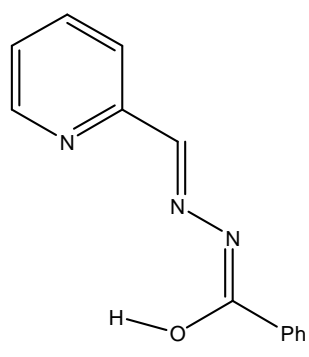

$\operatorname{Pr}^{3+}$ (Ref. 446)

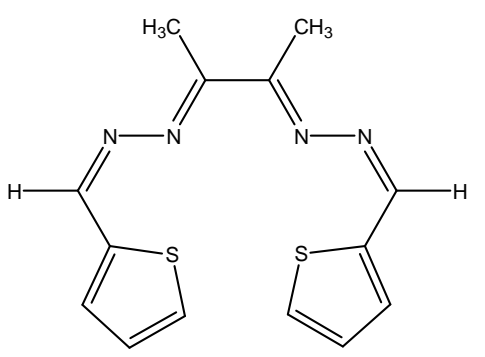

$\mathrm{Eu}^{3+}(\operatorname{Ref} .458)$<smiles>Cc1n[nH]/c(=N\N=c2/[nH]nc(C)c(=O)n2N)n(N)c1=O</smiles>

$\mathrm{Tb}^{3+}$ (Ref. 465)<smiles>Nc1cccc(/N=C/c2cccs2)n1</smiles>

$\mathrm{Lu}^{3+}$ (Ref. 485)

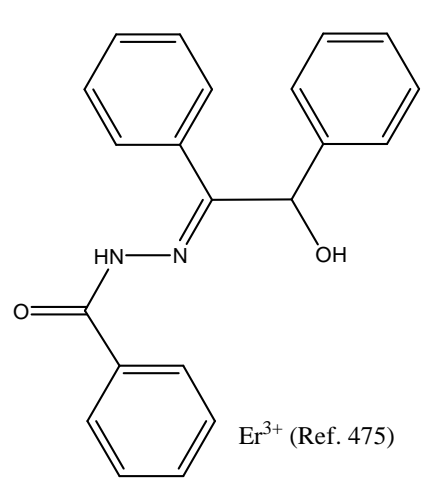<smiles>C/C=C(/C)c1csc(N/N=C/c2cccs2)n1</smiles>

$\mathrm{Tm}^{3+}$ (Ref. 478)<smiles>O=C(N/N=C\c1ccccc1O)c1cc2ccccc2cc1O</smiles>

$\mathrm{Yb}^{3+}$ (Ref. 480)

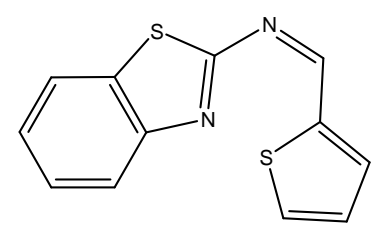

$\mathrm{Ho}^{3+}$ (Ref. 471) 
Ten reports on monohydrogen phosphate based on vanadyl salen, vanadyl salophen, macrocyclic dithioxamide, molybdenum acetylacetonate, oxo-molybdenum methylsalen, vanadylsalen complex (VS), Binuclear organotin, polymeric membrane electrodes, heterocylic macrocycles, and oxomolybdenum methyl-salen [107, 514-522].

Three reports on phosphate ion sensors based on bis(pentafluorobenzyl) tin(IV) dibromide, cobaltwire phosphate ion-selective, and organic tin compounds [523-525].

Two reports on cyanide membrane sensors based on aquacyanocobyrinic acid heptamethyl ester (ACCbs) reagent (orange color) at $\mathrm{pH} 9.5$ to give dicyarrocobester (DCCbs) (violet color) and thin electroplated membranes of silver chalcogenides [526,527].

About 30 reports on thiocyanide PVC membrane sensors based on ( $N, N$ '-bis-salicylidene-1,2ethylenediamine), 2.2-[(1,3-dimethyl-1,3-propanediylidene)dinitrilo]bis-benzenethiolato cadmium(II), butane-2,3-dione bis(salicylhydrazonato) zinc(II), dinuclear copper complex, N, $N$ '-ethylene-bis(4methylsalicylidineiminato)nickel(II), zinc-phthalocyanine complex, $[\mathrm{Cu}(\mathrm{L})]\left(\mathrm{NO}_{3}\right)_{2} \quad(\mathrm{~L}=4,7-$ bis(3aminopropyl)-1-thia-4,7-diazacyclononane), meso-tetraphenylporphyrin-rhodium(III), linear polyamines, poly(vinyl chloride) membrane electrode based on calix[4]arene, bis-[(3-ferrocenyl)-(2crotonic acid)] copper(II) complex [Cu(II)-BFCA], $N, N$ '-bis-(benzaldehyde)glycine metallic complexes of $\mathrm{Cu}(\mathrm{II}), \mathrm{Ni}(\mathrm{II}), \mathrm{Zn}$ (II) and $\mathrm{Co}(\mathrm{II})$, bis-taurine-salicylic binuclear copper(II) complex, tribenzyltin(IV) dithiocarbamate [Sn(IV)-TBDTB], rhodium(II) phthalocyanine (RhPc), a copper-1,8-dimethyl1,3,6,8,10,13-azacyclotetradecane complex, (octabromotetraphenylporphyrinato) manganese(III) chloride, nickel and iron phthalocyanines, tricoordinate Schiff's base copper(II) complex, 5,10,15,20tetrakis(2,4,6-trimethylphenyl)porphyrinatomanganese(III) chloride [ $\mathrm{Mn}(\mathrm{TMP}) \mathrm{Cl}]$ and 5,10,15,20tetrakis(2,6-dichlorophenyl)porphyrinatomanganese(III) chloride $\left[\mathrm{Mn}\left(\mathrm{C}_{18} \mathrm{TPP}\right) \mathrm{Cl}\right]$, two zinc(II) ions and two molecules of the bis- $N, O$-bidentate Schiff base 2,2'-[methylenebis(4,1-phenylenenitrilomethylidyne)]bisphenol, bisbenzoin-semitriethylenetetraamine binuclear cobalt(II) metallic complex [Co(II) $)_{2}$-BBSTA], $N, N^{\prime}$-bis-(furaldehyde)-1,2-phenylenediamine-dipicolyl copper(II) complex [Cu(II)-BFPD], benzaldehyde semicarbazone copper (II) complex [Cu(II)-BASA], bis-benzoinsemitriethylenetetriamine binuclear copper(II) [Cu(II)(2)-BBSTA], manganese(III) tetraphenylporphyrin derivatives, $\mathrm{Cu}$ (Il)-1,8-dimethyl-1,3,6,8,10,13-hexaazacyclotetradecane complex, nickel(II)azamacrocycle complex, and nickel(II)-1,4-,8,11,15,18,22,25-octabutoxyphthalocyanine [528-556].

One report on sulfite ion selective sensors based on bis-urea calix[4]diquinones [557]. More than ten reports for sulfate PVC membrane sensors based on schiff base complex of Zn(II), strontium Schiff's base complex (SS), zinc-Schiff base, $N, N$-ethylenebis(5-hydroxysalicylideneiminato) chromium(III) chloride, 1,3,5-triphenylpyrylium perchlorate, alpha,alpha'-bis( $N$ '-phenylthioureylene)$m$-xylene, and 2,5-diphenyl-1,2,4,5-tetraazabicyclo[2.2.1]heptane [558-569].

There are four reports on $\mathrm{F}^{-}$ion membrane sensors which are based on gallium(III)-Schiff base, organotin compounds, uranyl salophenes derivatives, $\mathrm{Zr}(\mathrm{IV}$ )-octaethylporphyrin (OEP) dichloride ( $\mathrm{Zr}(\mathrm{IV})[\mathrm{OEP}] \mathrm{Cl}-2)$ [570-573], three reports on $\mathrm{Cl}^{-}$electrodes based on ruthenium(III) Schiff's base, Schiff's base complex of cobalt(II), liquid polymer nano-PEBBLEs [574-576] and 11 reports on $\mathrm{ClO}_{3}{ }^{-}$ based on synthesized platinum(II) complexes, 1,4,7,10,13-penta(n-octyl)-1,4,7,10,13-pentaazacyclopentadecane, calcium bis [4-(1',1',3',3'-tetramethylbutyl)phenyl]phosphate, phosphadithiamacrocycle, octylammonium chloride, complex of uranyl, phosphorus(V)tetraphenylporphyrin, gold(I) organic 
complex, two nickel-hexaazamacrocycle complexes, some recently synthesized $\mathrm{Ni}(\mathrm{II})$ hexaazacyclotetradecane complexes, and doped poly(3,4-ethylenedioxythiophene) [577-587]; five reports on bromide based on iron(III)-salen (IS), Zn(II) macrocyclic complex, new mercury(II) complex, bis(4-hydroxyphenyl) 1,4-diaza-1,3-butadiene-Hg(II), benzo-derivative xanthenium bromide [588-592]; and 18 reports on iodide membrane sensors based on salen-Mn(II), Schiff's base complex of $\mathrm{Fe}$ (III), cerium-salen, [5,10,15,20-tetrakis(4- $N, N$-dimethylaminobenzene)porphyrinato] $\mathrm{Mn}$ (III) acetate, cobalt-salophen, silver-tin oxide, halide-selective receptor, urea derivative, porphyrin, titanium, acetylacetonate, homogeneous crystalline membrane, thiopyrilium ion derivative, PVC-DZT-Hg(II) compound, triphenyllead chloride, bis(1,3,4-thiadiazole) complexes of $\mathrm{Hg}(\mathrm{II})$, copper phthalocyanine, 1,4,8-tri( $n$-octyl)-1,4,8,11-tetraazacyclotetradecane complexes of a cyclam derivative, and copper (II) complex [593-610] and 23 reports for triiodide membrane sensors based on Schiff's base 2,2'[4,4'diphenylmethane bis(nitromethylidyne)] bisphenol, with copper(II) and Schiff's base 2,2'[4,4'diphenylmethane bis(nitromethylidyne)] bisphenol, with iron(III), bis(2-hydroxy-acetophenone)butane2,3-dihydrazone (ICT), bis(salicylaldehyde) ethylendiamine mercury(II) complex MS), N,N'-1,2propylene-bis-(5-methylsalicylidene iminato) copper(II), a charge-transfer complex, of (1,3diphenyldihydro-1H-imidazole-4,5-dione dioxide), with iodide, bis(2,4-dimethoxybenzaldehyde)butane-2,3-dihydrazone with iodine, 2-(((2)((E)-1-(2-hydroxyphenyl)methylidine) amino)phenyl)imino)methyl) phenol with iodine, complex of (1,3-diphenyldihydro-1H-imidazole)-4,5dione dioxime with iodine, complex of bis(2,4-dimethoxybenzaldehyde)butane-2,3-dihydrazone with iodine, bis- $N, O$-bidentate Schiff's base, bis(2-hydroxyacetophenone)butane-2,3-dihydrazone, 7,16dibenzyl-1,4,10,13-tetraoxa-7,16-diazacyclooctadecane, 2,4,6,8-tetraphenyl-2,4,6,8-tetraazabicyclo[3.3.0] octane, 1,6-bis( $N, N$-diethylthiocarbamoylimino)-1,6-diphenyl-2,5-dithiahexane, mercury-salen, phenothiazine derivatives, tetra( $p$-chlorophenyl)porphyrinato manganese(III) acetate, tetrachlorophenylporphyrinato manganese(III) acetate, ketoconazole-triiodide, two different charge-transfer complexes and amino crown ether, clotrimazole-triiodide ion, complex of iodine and bis(2hydroxyacetophenone)butane-2,3-dihydrazone, complex of 2-(((2-(((E)-1-(2-hydroxyphenyl) methylidine)amino)phenyl)imino)methyl) phenol [88, 611-630]. There is also one report on periodide membrane sensors based on metaperiodate bis(triphenylphosphoranylidene)ammonium [631] and one report for arsenite PVC membrane sensor based on 5,10,15,20-tetrakis (4-methoxyphenyl) porphyrinato cobalt(II) [632].

The statistical diagram of the reported potentiometric membrane sensors based on non-conducting polymer for inorganic anions is shown in Figure 17. 
Figure 17. The statistical diagram of the reported potentiometric membrane sensors based on conducting and non-conducting polymer for inorganic anions.

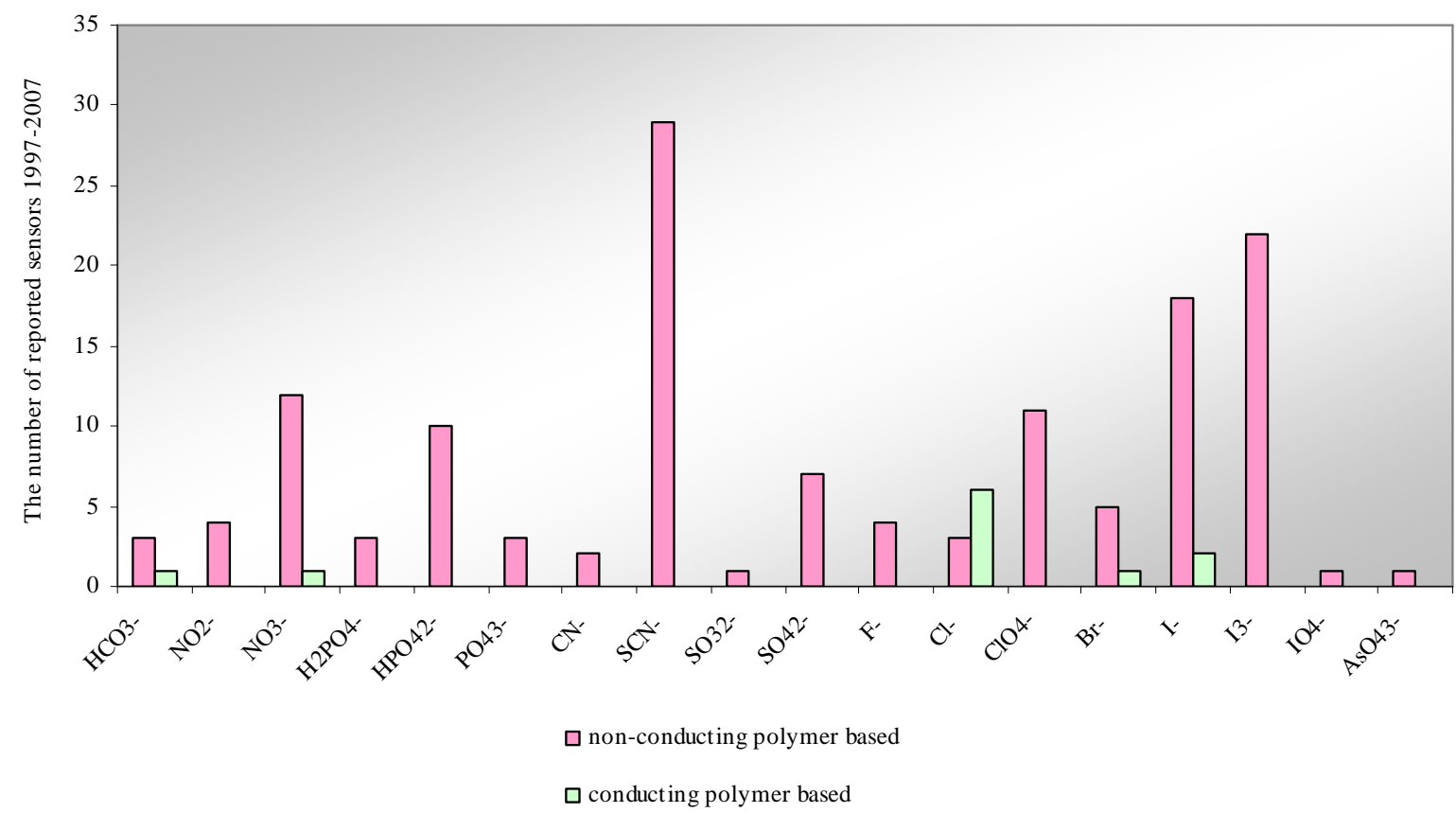

Table 8 shows the characterization and properties of the reported inorganic anion membrane sensors

Table 8. Characterization of a number of reported ion selective sensors based on nonconducting polymers for inorganic anions.

\begin{tabular}{|c|c|c|c|c|c|}
\hline Anion & Ionophore & $\begin{array}{c}\text { Slope } \\
\left(\mathbf{m V} \text { decade }^{-1}\right)\end{array}$ & $\begin{array}{l}\text { Linear Range } \\
\text { (M) }\end{array}$ & $\begin{array}{l}\text { The most } \\
\text { interfering ions } \\
\left(\log K_{\mathrm{Sel}}>-2\right) \\
\end{array}$ & Ref. \\
\hline $\mathrm{HCO}_{3}^{-}$ & $\begin{array}{l}\text { long chain S-akyl } \\
\text { diphenylthiocarbazone. }\end{array}$ & -54 & $10^{-2}-10^{-5}$ & - & 493 \\
\hline $\mathrm{NO}_{2}^{-}$ & $\begin{array}{l}\text { (tetraphenylporphyrinato) } \\
\text { cobalt(III) acetate }\end{array}$ & $-58.4-60.8$ & $\begin{array}{l}1.0 \times 10^{-6}-1.0 \times 10^{-1} \\
\text { and } 5.0 \times 10^{-8}- \\
5.0 \times 10^{-2}\end{array}$ & $\mathrm{~F}^{-}$ & 496 \\
\hline $\mathrm{NO}_{3}^{-}$ & $\begin{array}{l}N, N, N \text {-triallylleucine betaine } \\
\text { chloride }\end{array}$ & -59.1 & $1 \times 10^{-6}-2.25 \times 10^{-2}$ & - & 507 \\
\hline $\begin{array}{l}\mathrm{HPO}_{4}{ }^{2-}-1 \\
\mathrm{HPO}_{4}{ }^{2-}-2\end{array}$ & $\begin{array}{l}\text { molybdenum acetylacetonate } \\
\text { oxo-molybdenum methyl- } \\
\text { salen }\end{array}$ & $\begin{array}{l}-29.5 \\
-28.6\end{array}$ & $\begin{array}{l}1.0 \times 10^{-1}-1.0 \times 10^{-7} \\
1.0 \times 10^{-1}-4.0 \times 10^{-7}\end{array}$ & - & $\begin{array}{l}516 \\
517\end{array}$ \\
\hline $\mathrm{PO}_{4}{ }^{3-}$ & $\begin{array}{l}\text { bis(pentafluorobenzyl) } \\
\text { tin(IV) dibromide }\end{array}$ & -70.8 & $10^{-5}-10^{-1}$ & - & 523 \\
\hline $\mathrm{CN}^{-}$ & $\begin{array}{l}\text { thin electroplated membranes } \\
\text { of silver chalcogenides }\end{array}$ & -90 & $10^{-6}-10^{-2}$ & - & 527 \\
\hline
\end{tabular}


Table 8 .Cont

\begin{tabular}{|c|c|c|c|c|c|}
\hline Anion & Ionophore & $\begin{array}{c}\text { Slope } \\
\left(\mathrm{mV} \text { decade }^{-1}\right)\end{array}$ & $\begin{array}{c}\text { Linear Range } \\
\text { (M) }\end{array}$ & $\begin{array}{l}\text { The most } \\
\text { interfering ions } \\
\left(\log K_{\mathrm{Sel}}>-2\right) \\
\end{array}$ & Ref. \\
\hline $\mathrm{SCN}^{-}-1$ & $\begin{array}{l}\text { (octabromotetraphenylporphy } \\
\text { rinato)manganese(III) } \\
\text { chloride }\end{array}$ & -58.3 & $4.8 \times 10^{-7}-1.0 \times 10^{-}$ & - & 544 \\
\hline $\mathrm{SCN}^{-}-2$ & $\begin{array}{l}\text { manganese(III) } \\
\text { tetraphenylporphyrin } \\
\text { derivatives }\end{array}$ & -59.5 & $10^{-7}-10^{-1}$ & - & 553 \\
\hline $\mathrm{SCN}^{-}-3$ & $\begin{array}{l}\text { nickel(II)-azamacrocycle } \\
\text { complex }\end{array}$ & -57.8 & $1.0 \times 10^{-7}-1.0 \times 10^{-}$ & - & 555 \\
\hline $\mathrm{SO}_{3}^{2-}$ & bis-urea calix[4]diquinones & -51.5 & $6.0 \times 10^{-5}-1.0 \times 10^{-2}$ & $\mathrm{ClO}_{4}^{-}$ & 557 \\
\hline $\mathrm{SO}_{4}^{2-}-1$ & $\begin{array}{l}\text { strontium Schiff's base } \\
\text { complex (SS) }\end{array}$ & -29.2 & $10^{-2}-10^{-6}$ & $\mathrm{SO}_{3}{ }^{2-}, \mathrm{CO}_{3}{ }^{2-}, \mathrm{Cl}^{-}$ & 559 \\
\hline $\mathrm{SO}_{4}{ }^{2-}-2$ & zinc-Schiff base & -29.2 & $10^{-2}-10^{-6}$ & - & 560 \\
\hline $\mathrm{SO}_{4}{ }^{2-}-3$ & $\begin{array}{l}\text { alpha,alpha'-bis( }\left(N^{\prime} \text {-phenyl- }\right. \\
\text { thioureylene)-m-xylene }\end{array}$ & -29.6 & $10^{-6}-10^{-2}$ & $\mathrm{SCN}^{-}, \mathrm{Br}^{-}, \mathrm{NO}_{2}^{-}$ & 563 \\
\hline $\mathrm{F}^{-}$ & organotin compounds & -62.7 & $1.0 \times 10^{-6}-1.0 \times 10^{-1}$ & - & 571 \\
\hline $\mathrm{Cl}^{-}$ & ruthenium(III) Schiff's base & -54.5 & $1.0 \times 10^{-1}-3.0 \times 10^{-6}$ & - & 574 \\
\hline $\begin{array}{l}\mathrm{ClO}_{4}^{-}-1 \\
\mathrm{ClO}_{4}^{-}-2\end{array}$ & $\begin{array}{l}\text { complex of uranil } \\
\text { some recently synthesized } \\
\mathrm{Ni}(\mathrm{II}) \text {-hexaazacyclo- } \\
\text { tetradecane complexes }\end{array}$ & $\begin{array}{l}-60.6 \pm 1.0 \\
-59.3\end{array}$ & $\begin{array}{l}1.0 \times 10^{-6}-1.0 \\
10^{-1}-5.0 \times 10^{-7}\end{array}$ & $\begin{array}{l}- \\
-\end{array}$ & $\begin{array}{l}584 \\
586\end{array}$ \\
\hline $\begin{array}{l}\mathrm{Br}^{-}-1 \\
\mathrm{Br}^{-}-2\end{array}$ & $\begin{array}{l}\text { iron(III)-salen (IS) } \\
\mathrm{Zn}(\mathrm{II}) \text { macrocyclic complex }\end{array}$ & $\begin{array}{l}-59.0 \\
-59.2\end{array}$ & $\begin{array}{l}7.0 \times 10^{-6}-1.0 \times 10^{-1} \\
2.2 \times 10^{-6}-1.0 \times 10^{-1}\end{array}$ & $\begin{array}{l}\mathrm{SCN}^{-}, \mathrm{I}^{-}, \mathrm{Cl}^{-} \\
-\end{array}$ & $\begin{array}{l}588 \\
589\end{array}$ \\
\hline $\mathrm{I}^{-}-1$ & & -58.9 & $\begin{array}{l}5.0 \times 10^{-7}- \\
1.0 \times 10^{-1}\end{array}$ & - & 597 \\
\hline $\mathrm{I}^{-}-2$ & thiopyrilium ion derivative & -60 & $8.0 \times 10^{-7}-1.0 \times 10^{-1}$ & - & 604 \\
\hline $\mathrm{I}^{-}-3$ & $\begin{array}{l}\text { bis(1,3,4-thiadiazole }) \\
\text { complexes of } \mathrm{Hg}(\mathrm{II})\end{array}$ & -59.0 & $2.0 \times 10^{-8}-2.0 \times 10^{-2}$ & - & 607 \\
\hline $\mathrm{I}_{3}^{-}-1$ & $\begin{array}{l}\text { bis (salicylaldehyde) } \\
\text { ethylendiamine mercury(II) } \\
\text { complex MS) }\end{array}$ & -59.0 & $5.0 \times 10^{-8}-1.0 \times 10^{-2}$ & - & 613 \\
\hline $\mathrm{I}_{3}-2$ & $\begin{array}{l}\text { a charge-transfer complex } \\
\text { of }(1,3 \text {-diphenyldihydro- } 1 \mathrm{H}- \\
\text { Imidazole-4,5-dione dioxide } \\
\text { with Iodide }\end{array}$ & -59.3 & $10^{-7}-10^{-1}$ & - & 615 \\
\hline $\mathrm{I}_{3}-3$ & $\begin{array}{l}\text { 2-((( 2)((E)-1-( 2-hydroxy- } \\
\text { phenyl) methylidine)amino)- } \\
\text { phenyl)imino) methyl) phenol } \\
\text { with iodine ( CTC) }\end{array}$ & -59 & $5.0 \times 10^{-8}-1.0 \times 10^{-2}$ & - & 88 \\
\hline $\mathrm{I}_{3}{ }^{-} 4$ & mercury-salen & $-59.0 \pm 0.5$ & $\begin{array}{l}5.0 \times 10^{-8}- \\
1.0 \times 10^{-2}\end{array}$ & - & 623 \\
\hline
\end{tabular}


Table 8. Cont.

\begin{tabular}{|c|c|c|c|c|c|}
\hline Anion & Ionophore & $\begin{array}{c}\text { Slope } \\
\left(\mathrm{mV} \mathrm{decade}^{-1}\right)\end{array}$ & $\begin{array}{c}\text { Linear Range } \\
\text { (M) }\end{array}$ & $\begin{array}{l}\text { The most } \\
\text { interfering ions } \\
\left(\log K_{\text {Sel }}>-2\right)\end{array}$ & Ref. \\
\hline $\mathrm{IO}_{4}^{-}$ & $\begin{array}{l}\text { metaperiodate } \\
\text { bis(triphenylphosphoranylide } \\
\text { ne) ammonium }\end{array}$ & -60.1 & $8.0 \times 10^{-3}-2.7 \times 10^{-1}$ & - & 631 \\
\hline Arsenite & $\begin{array}{l}\text { PVC based } 5,10,15,20- \\
\text { tetrakis (4-methoxyphenyl) } \\
\text { porphyrinato cobalt(II) }\end{array}$ & -28.8 & $7.9 \times 10^{-5}-1.0 \times 10^{-1}$ & - & 632 \\
\hline
\end{tabular}

Chemical structures of some above ionophores which are used in construction of non-conducting polymer ISEs for inorganic anions are shown in Figure 18.

Figure 18. Some structures of the suitable ionophores used in construction of some anion membrane sensors.

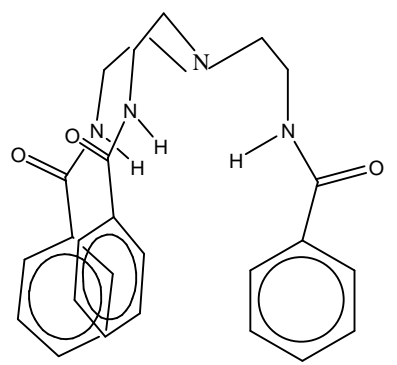

$\mathrm{NO}_{3}^{-}$(Ref. 505)

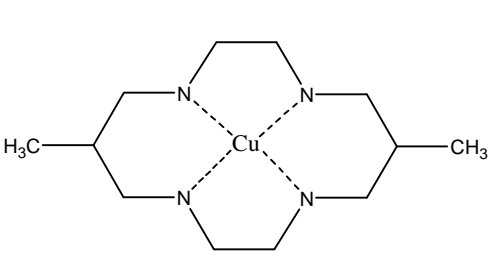

$\mathrm{SCN}^{-}$(Ref. 544)

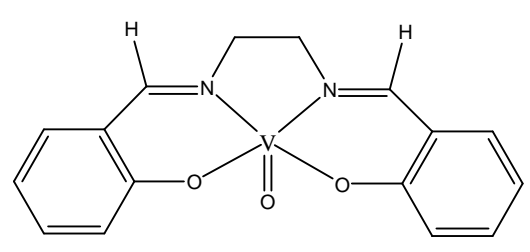

$\mathrm{HPO}_{4}{ }^{2-}$ (Ref. 518)

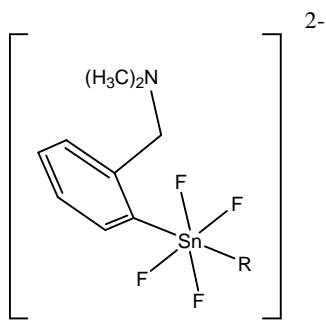

$\mathrm{F}^{-}($Ref. 571)<smiles>c1ccc(N2CN3CN2CN3c2ccccc2)cc1</smiles>

$\mathrm{SO}_{4}{ }^{2-}$ (Ref. 563)

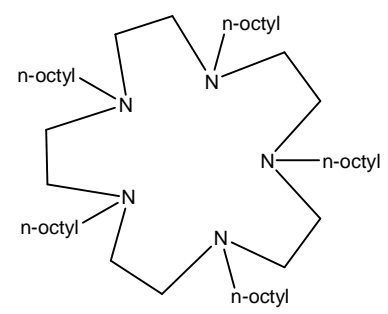

$\mathrm{ClO}_{4}^{-}$(Ref. 578)

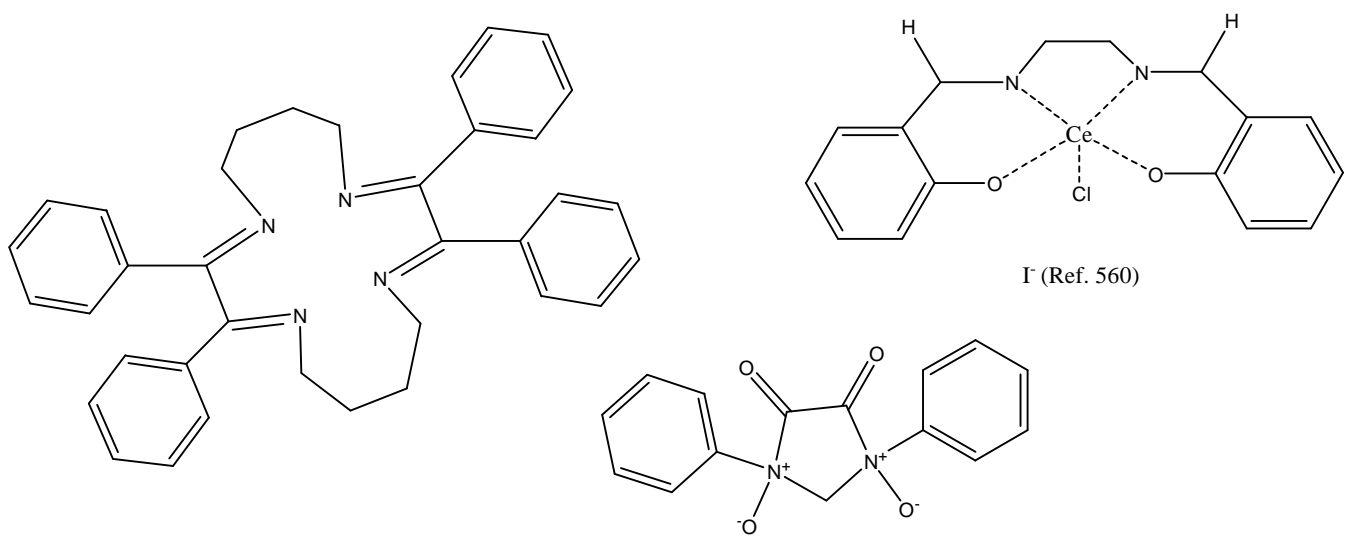

$\mathrm{Br}^{-}$(Ref. 589) 


\section{Conclusions}

The main problem with symmetric ion selective PVC membrane electrodes is the leakage of the internal solution to the outer surface of the membrane, causing changes in the surface potential. Therefore, the detection limit of this kind of electrode is about $10^{-6} \mathrm{M}$. Since the lifetime of the plasticized PVC membranes is affected by leaching of the membrane components from the membrane into the sample solution, many efforts have been made to achieve plasticizer-free membranes and covalently bound ion recognition sites, in which case CPs also offer unique possibilities.

Construction of all-solid-state ISEs that do not require internal filling solutions is a common approach, which necessitates careful designs of the solid contact between the ion-selective membrane and the electronic conductor. These devices, however, suffered from the ill-defined transduction of the ionically conducting ion-selective membrane and the electronic conductor, which made them rather instable. Scientists solved overcame this simply by using a conducting polymer instead of the liquid internal electrolyte of the classical ISE. Conducting polymers are able to form an ohmic contact to materials of high work functions, like carbon, gold and platinum. Secondly, due to the solubility of several CPs, they can be deposited from solutions. Thirdly, they have ability to being electroactive materials of mixed electronic and ionic conductivity, which gives them the unique potential to transduce ionic signals into electronic ones.

These kinds of electrodes have lower detection limits, high mechanical resistance and reproducibility rather to the PVC based membrane electrodes. Also, they can be easily miniaturized to yield solid state ion-selective microelectrodes.

However, the diversity of the electrodes having conducting polymer in comparison to the common PVC based membrane electrodes is very low.

\section{References and Notes}

1. Bakker, E.; Buhlmann, P.; Pretsch, E. Carrier-based ion-selective electrodes and bulk optodes. 1. General characteristics. Chem. Rev. 1997, 97, 3083-3132.

2. Bakker, E.; Pretsch, E. Potentiometric sensors for trace-level analysis. Trends Anal. Chem. 2005, 24, 199-207.

3. Ganjali, M. R.; Norouzi, P.; Rezapour, M.; Faridbod, F.; Pourjavid, M. R. Supramolecular Based Membrane Sensors. Sensors 2006, 6, 1018-1086.

4. Ganjali, M.R.; Norouzi, P.; Faridbod, F.; Rezapour, M.; Pourjavid, M.R. One Decade of Research on Ion-Selective Electrodes in Iran (1996-2006). J. Iran. Chem. Soc. 2007, 4, 1-29.

5. Ganjali, M. R.; Dinarvand, R.; Norouzi, P.; Faridbod, F. Ion Recognition: Application of Symmetric and Asymmetric Schiff Bases and Their Complexes for the Fabrication of Cationic and Anionic Membrane Sensors to Determine Ions in Real Samples. Comb. Chem.High Throughput Screen. 2007, 10, 527-546.

6. Faridbod, F.; Ganjali, M. R.; Dinarvand, R.; Norouzi, P. The fabrication of potentiometric membrane sensors and their applications. Afr. J. Biotechnol. 2007, 6, 2960-2987. 
7. Ganjali, M. R.; Norouzi, P.; Rezapour, M. Encyclopedia of Sensors, Potentiometric Ion Sensors. American Scientific Publisher (ASP): Stevenson Ranch, CA, USA, 2006, 8, 197-288.

8. Sokalski, T.; Ceresa, A.; Zwickl, T.; Pretsch, E. J. Large improvement of the lower detection limit of ion-selective polymer membrane electrodes. J. Amer. Chem. Soc. 1997, 119, 1134711348.

9. Bobacka, J.; Ivaska, A.; Lewenstam, A. Potentiometric Ion Sensors Based on Conducting Polymers. Electroanalysis 2003, 15, 366-374.

10. Bobacka, J.; Lindfors, T.; Lewenstam, A.; Ivaska, A. All-solid-state ion sensors, usiong conducting polymers as ion-to-electron transducers. Am. Lab. 2004, 36, 12-20.

11. Bobacka, J. Conducting Polymer-Based Solid-State Ion-Selective Electrodes. Electroanalysis 2006, 18, 7-18

12. Vigassy, T.; Huber, C. G.; Wintringer, R.; Pretsch, E. Monolithic capillary-based ion-selective electrodes. Anal. Chem. 2005, 77, 3966-3970.

13. Nikolskii, B. P.; Materova, E. A. Ion-Sel. Electrode Rev. 1985, 7, 3.

14. Cattrall, R. W.; Freiser, H. Anal. Chem. 1971, 43, 1905.

15. Lindner, E.; Cosofret,V. V.; Ufer, S.; Johnson, T. A.; Ash, R. B.; Nagle, H. T.; Neuman, M. R.; Buck, R. P. In-Vivo and In-Vitro testing of microelectronically fabricated planar sensors designed for application in cadiology. Fresen. J. Anal. Chem. 1993, 346, 584-588.

16. Fibbioli, M.; Bandyopadhyay, K.; Liu, S-G.; Echegoyen, L.; Enger, O.; Diederich, F.; Bühlmann, P.; Pretsch, E. Redox-active self-assembled monolayers as novel solid contacts for ion-selective electrodes. Chem. Commu. 2000, 339.

17. Bobacka, J. Potential stability of all-solid-state ion-selective electrodes using conducting polymers as ion-to-electron transducers. Anal. Chem. 1999, 71, 4932-4937.

18. Rahman, A.; Kumar, P.; Park, D. S.; Shim, Y. B. Electrochemical Sensors Based on Organic Conjugated Polymers. Sensors 2008, 8, 118-141.

19. Rahman, A.; Lee, K. S.; Park, D. S.; Won, M. S.; Shim, Y. B. An amperometric bilirubin biosensor based on a conductive poly-terthiophene-Mn(II) complex. Biosens. Bioelectron. 2008, 23, 857-864.

20. Kum, M. C.; Joshi, K. A.; Chen, W. Biomolecules-carbon nanotubes doped conducting polymer nanocomposites and their sensor application. Talanta 2007, 74, 370-375.

21. Ramanavicius, A.; Ramanaviciene, A.; Malinauskas, A. Electrochemical sensors based on conducting polymer- polypyrrole. Electrochim. Acta 2006, 51, 6025-6037.

22. Rahman, M. A.; Park, D. S.; Chang, S. C.; McNeil, C. J.; Shim, Y. B. The biosensor based on the pyruvate oxidase modified conducting polymer for phosphate ions determinations. Biosens. Bioelectron. 2006, 21, 1116-1124.

23. Janata, J.; Josowicz, M. Conducting polymers in electronic chemical sensors. Nat. Mater. 2003, 2, 19-24.

24. Bobacka, J. In Encyclopedia of Sensors; Grimes, C. A.; Dickey, E.; Pishko, M. V. Eds.; American Scientific Publishers: Stevenson Ranch, CA, USA, 2006.

25. Ramanaviciene, A.; Ramanavicius, A. Application of polypyrrole for the creation of immunosensors Crit. Revi. Anal. Chem. 2002, 32, 245-252. 
26. Gyurcscnyi R. E.; Nybck, A-S.; Tth, K.; Nagy, G.; Ivaska. A. Novel, polypyrrole based all-solidstate potassium-selective microelectrodes. Analyst 1998, 123, 1339-44.

27. Bobacka, J.; Lindfors, T.; McCarrick, M.; Ivaska, A.; Lewenstam, A. Single-piece all-solid-state ion-selective electrode. Anal. Chem. 1995, 67, 3819-3823.

28. Migdalski, J.; Blaz, T.; Lewenstam, A. Conducting polymer-based ion-selective electrodes. Anal. Chim. Acta 1996, 322, 141-149.

29. Migdalski, J, Blaz, T.; Kowalski, Z.; Lewenstam, A. Nonclassical potentiometric indicator electrodes with dual sensitivity. Electroanalysis 1999, 11, 735-743.

30. Blaz, T.; Migdalski, J.; Lewenstam, A. Polypyrrole-calcion film as a membrane and solid-contact in an indicator electrode for potentio-metric titrations. Talanta 2000, 52, 319-328.

31. Bobacka, J.; Ivaska, A.; Lewenstam, A. Plasticizer-free all-solid-state potassium-selective electrode based on poly (3-octylthiophene) and valinomycin. Anal. Chim. Acta 1999, 385, 195202.

32. Sjberg, P.; Bobacka, J.; Lewenstam, A.; Ivaska, A. All-solid-state chlo-ride-selective electrode based on poly (3-octylthiophene) and tridode-cylmethylammonium chloride. Electroanalysis 1999, 11, 821-824.

33. Lindfors, T.; Ivaska, A. All-solid-state calcium-selective electrode prepared of soluble electrically conducting polyaniline and di(2-ethylhexyl)phosphate with ETH1001 as neutral carrier. Anal. Chim. Acta 2000, 404, 101-110.

34. Lindfors, T.; Ivaska, A. All-solid-state calcium-selective electrode prepared of soluble electrically conducting polyaniline and di(2-ethylhexyl)phosphate with tetraoctylammonium chloride as cationic additive. Anal. Chim. Acta 2000, 404, 111-119.

35. Lindfors, T.; Ivaska, A. Calcium-selective electrode based on polyani-line functionalized with bis[4(1,1,3,3-tetramethylbutyl)phenyl]-phosphate. Anal. Chim. Acta 2001, 437, 171-182.

36. Lindino, C. A.; Bulhoes, L. O. S. The potentiometric response of chemically modified electrodes. Anal. Chim. Acta 1996, 334, 317-322.

37. Faria, R. C.; Bulhoes, L. O. S. Hydrogen ion selective electrode based on poly(1aminoanthracene) film. Anal. Chim. Acta 1998, 377, 21-27.

38. Masalles, C.; Borros, S.; Vinas, C.; Teixidor, F. Simple PVC-PPy electrode for $\mathrm{pH}$ measurement and titrations. Anal. Bioanal. Chem. 2002, 372, 513-518.

39. Lindfors, T.; Ivaska, A. $\mathrm{pH}$ sensitivity of polyaniline and its substituted derivatives. $J$. Electroanal. Chem. 2002, 531, 43-52.

40. Lindfors, T.; Ervela, S.; Ivaska, A. Polyaniline as $\mathrm{pH}$-sensitive component in plasticized PVC membranes. J. Electroanal. Chem. 2003, 560, 69-78.

41. Zine, N.; Bausells, J.; Ivorra, A.; Aguilo, J.; Zabala, M.; Teixidor, F.; Masalles, C.; Vinas, C.; Errachid, A. Hydrogen-selective microelectrodes based on silicon needles. Sens. Actuators B 2003, 91, 76-82.

42. Michalska, A.; Maksymiuk, K. Counter-ion influence on polypyrrole potentiometric $\mathrm{pH}$ sensitivity. Microchim. Acta 2003, 143, 163-175.

43. Kaden, H.; Jahn, H.; Berthold, M. Study of the glass/polypyrrole interface in an all-solid-state $\mathrm{pH}$ sensor. Solid State Ionics 2004, 169, 129-133. 
44. Ge, C. H.; Armstrong, N. R.; Saavedra, S. S. PH-sensing properties of poly(aniline) ultrathin films self-assembled on indium-tin oxide. Anal. Chem. 2007, 79, 1401-1410.

45. Cadogan, A.; Gao, Z. Q.; Lewenstam, A.; Ivaska, A.; Diamond, D. All-Solid-State SodiumSelective Electrode Based on a Calixarene Ionophore in a Poly(Vinyl Chloride) Membrane with a Polypyrrole Solid Contact. Anal. Chem. 1992, 64, 2496-2501.

46. Pawlowski, P.; Michalska, A.; Maksymiuk, K. Galvanostatic polarization of all-solid-state K+selective electrodes with polypyrrole ion-to-electron transducer. Electroanalysis 2006, 18, 13391346.

47. Bobacka, J.; Ivaska, A.; Lewenstam, A. Plasticizer-free all-solid-state potassium-selective electrode based on poly(3-octylthiophene) and valinomycin. Anal. Chim. Acta 1999, 385, 195202.

48. Gyurcsanyi, R. E.; Nyback, A. S.; Toth, K.; Nagy, G.; Ivaska, A. Novel polypyrrole based allsolid-state potassium-selective microelectrodes. Analyst 1998, 123, 1339-1344.

49. Lindfors, T.; Ivaska, A. Stability of the inner polyaniline solid contact layer in all-solid-state $\mathrm{K}^{+}$selective electrodes based on plasticized poly(vinyl chloride). Anal. Chem. 2004, 76, 4387-4394.

50. Vazquez, M.; Danielsson, P.; Bobacka, J.; Lewenstam, A.; Ivaska, A. Solution-cast films of poly(3,4-ethylenedioxythiophene) as ion-to-electron transducers in all-solid-state ion-selective electrodes. Sens. Actuators B 2004, 97, 182-189.

51. Vazquez, M.; Bobacka, J.; Ivaska, A.; Lewenstam, A. Small-volume radial flow cell for all-solidstate ion-selective electrodes. Talanta 2004, 62, 57-63.

52. Gyurcsanyi, R. E.; Rangisetty, N.; Clifton, S.; Pendley, B. D.; Lindner, E. Microfabricated ISEs: critical comparison of inherently conducting polymer and hydrogel based inner contacts. Talanta 2004, 63, 89-99.

53. Toczylowska, R.; Pokrop, R.; Dybko, A.; Wroblewski, W. Planar potentiometric sensors based on $\mathrm{Au}$ and $\mathrm{Ag}$ microelectrodes and conducting polymers for flow-cell analysis. Anal. Chim. Acta 2005, 540, 167-172.

54. Pandey, P. C.; Prakash, R.; Singh, G.; Tiwari, I.; Tripathi, V. S. Studies on polycarbazolemodified electrode and its applications in the development of solid-state potassium and copper(II) ion sensors. J. Appl. Polym. Sci. 2000, 75, 1749-1759.

55. Paczosa-Bator, B.; Peltonen, J.; Bobacka, J.; Lewenstam, A. Influence of morphology and topography on potentiometric response of magnesium and calcium sensitive PEDOT films doped with adenosine triphosphate (ATP). Anal. Chim. Acta 2006, 555, 118-127.

56. Lindfors, T.; Ivaska, A. Calcium-selective electrode based on polyaniline functionalized with bis[4-(1,1,3,3-tetramethylbutyl)phenyl]phosphate. Anal. Chim. Acta 2001, 437, 171-182.

57. Lindfors, T.; Ivaska, A. All-solid-state calcium-selective electrode prepared of soluble electrically conducting polyaniline and di(2-ethylhexyl)phosphate with tetraoctylammonium chloride as cationic additive. Anal. Chim. Acta 2000, 404, 111-119.

58. Michalska, A. Improvement of analytical characteristic of calcium selective electrode with conducting polymer contact. The role of conducting polymer spontaneous charge transfer processes and their galvanostatic compensation. Electroanalysis 2005, 17, 400-407. 
59. Lindfors, T.; Ivaska, A. All-solid-state calcium-selective electrode prepared of soluble electrically conducting polyaniline and di(2-ethylhexyl)phosphate with ETH1001 as neutral carrier. Anal. Chim. Acta 2000, 404, 101-110.

60. Bobacka, J.; McCarrick, M.; Lewenstam, A.; Ivaska, A. All-Solid-State Poly(Vinyl Chloride) Membrane Ion-Selective Electrodes with Poly(3-Octylthiophene) Solid Internal Contact. Analyst 1994, 119, 1985-1991.

61. Michalska, A.; Maksymiuk, K. All-plastic, disposable, low detection limit ion-selective electrodes. Anal. Chim. Acta 2004, 523, 97-105.

62. Ragini, P. V.; Rizvi, G. H.; Contractor, A. Q.; Manchanda, V. K. Conducting polymer based electrochemical sensor for strontium in nitric acid medium. J. Indian Chem. Soc. 2002, 79, 949952.

63. Kisiel, A.; Michalska, A.; Maksymiuk, K. Plastic reference electrodes and plastic potentiometric cells with dispersion cast poly(3,4-ethylenedioxythiophene) and poly(vinyl chloride) based membranes. Bioelectrochemistry 2007, 71, 75-80.

64. Sutter, J.; Lindner, E.; Gyurcsanyi, R. E.; Pretsch, E. A polypyrrole-based solid-contact Pb2+selective PVC-membrane electrode with a nanomolar detection limit. Anal. Bioanal. Chem. 2004, 380, 7-14.

65. Ocypa, M.; Michalska, A.; Maksymiuk, K. Accumulation of $\mathrm{Cu}(\mathrm{II})$ cations in poly(3,4ethylenedioxythiophene) films doped by hexacyanoferrate anions and its application in $\mathrm{Cu}^{2+}$ selective electrodes with PVC based membranes. Electrochim. Acta 2006, 51, 2298-2305.

66. Migdalski, J.; Blaz, T.; Zralka, B.; Lewenstam, A. Galvanic cell without liquid junction for potentiometric determination of copper. Anal. Chim. Acta 2007, 594, 204-210.

67. Bobacka, J.; Lahtinen, T.; Koskinen, H.; Rissanen, K.; Lewenstam, A.; Ivaska, A. Silver ionselective electrodes based on pi-coordinating ionophores without heteroatoms. Electroanalysis 2002, 14, 1353-1357.

68. Vazquez, M.; Bobacka, J.; Ivaska, A. Potentiometric sensors for $\mathrm{Ag}^{+}$based on poly(3octylthiophene) (POT). J. Solid State Electrochem. 2005, 9, 865-873.

69. Vazquez, M.; Bobacka, J.; Luostarinen, M.; Rissanen, K.; Lewenstam, A.; Ivaska, A. Potentiometric sensors based on poly(3,4-ethylenedioxythiophene) (PEDOT) doped with sulfonated calix[4]arene and calix[4]resorcarenes. J. Solid State Electrochem. 2005, 9, 312-319.

70. Mousavi, Z.; Bobacka, J.; Lewenstam, A.; Ivaska, A. Response mechanism of potentiometric $\mathrm{Ag}^{+}$sensor based on poly(3,4-ethylenedioxythiophene) doped with silver hexabromocarborane. $J$. Electroanal. Chem. 2006, 593, 219-226.

71. Mousavi, Z.; Bobacka, J.; Ivaska, A. Potentiometric $\mathrm{Ag}^{+}$sensors based on conducting polymers: A comparison between poly(3,4-ethylenedioxythiophene) and polypyrrole doped with sulfonated calixarenes. Electroanalysis 2005, 17, 1609-1615.

72. Pandey, P. C.; Singh, G.; Srivastava, P. K. Electrochemical synthesis of tetraphenylborate doped polypyrrole and its applications in designing a novel zinc and potassium ion sensor. Electroanalysis 2002, 14, 427-432.

73. Khan, A. A.; Alam, M. M. New and novel organic-inorganic type crystalline 'polypyrrolel/polyantimonic acid' composite system: preparation, characterization and analytical 
applications as a cation-exchange material and $\mathrm{Hg}$ (II) ion-selective membrane electrode. Anal. Chim. Acta 2004, 504, 253-264.

74. Song, F. Y.; Ha, J. H.; Park, B.; Kwak, T. H.; Kim, I. T.; Nam, H.; Cha, G. S. All-solid-state carbonate-selective electrode based on a molecular tweezer-type neutral carrier with solventsoluble conducting polymer solid contact. Talanta 2002, 57, 263-270.

75. Hutchins, R. S.; Bachas, L. G. Nitrate-Selective Electrode Developed by Electrochemically Mediated Imprinting Doping of Polypyrrole. Anal. chem. 1995, 67, 1654-1660.

76. Atta, N. F.; Galal, A.; Mark, H. B.; Yu, T.; Bishop, P. L. Conducting polymer ion sensor electrodes III. Potentiometric sulfide ion selective electrode. Talanta 1998, 47, 987-999.

77. Shishkanova, T. V.; Sapurina, I.; Stejskal, J.; Kral, V.; Volf, R. Ion-selective electrodes: Polyaniline modification and anion recognition. Anal. Chim. Acta 2005, 553, 160-168.

78. Sjoberg, P.; Bobacka, J.; Lewenstam, A.; Ivaska, A. All-solid-state chloride-selective electrode based on poly(3-octylthiophene) and tridodecylmethylammonium chloride. Electroanalysis 1999, 11, 821-824.

79. Sjoberg-Eerola, P.; Bobacka, J.; Sokalski, T.; Mieczkowski, J.; Ivaska, A.; Lewenstam, A. Allsolid-state chloride sensors with poly(3-octylthiopene) matrix and trihexadecylmethylammonium chlorides as an ion exchanger salt. Electroanalysis 2004, 16, 379-385.

80. Michalska, A.; Walkiewicz, S.; Maksymiuk, K.; Hall, E. A. H. Potentiometric responses of poly(pyrrole) films surface modified by Nafion. Electroanalysis 2002, 14, 1236-1244.

81. Michalska, A.; Dumanska, J.; Maksymiuk, K. Lowering the detection limit of ion-selective plastic membrane electrodes with conducting polymer solid contact and conducting polymer potentiometric sensors. Anal. Chem. 2003, 75, 4964-4974.

82. Dominguez-Ballart, M.; Bachas, L. G.; Salvado, V. Potentiometric chloride-selective electrodes based on polypyrrole: preparation and membrane characterization by SEM and EDX. Quim. Anal. (Barcelona) 2000, 19, 49-56.

83. Michalska, A.; Galuszkiewicz, A.; Ogonowska, M.; Ocypa, M.; Maksymiuk, K. PEDOT films: multifunctional membranes for electrochemical ion sensing. J. Solid State Electrochem. 2004, 8, 381-389.

84. Wang, Z.; Zhang, H. S.; Mark, H. B.; Rubinson, J. F. Fabrication of a poly(methylthiophenemethylpyrrole) copolymer $\mathrm{Br}$ - sensor and its application in direct potentiometry and potentiometric titration. Anal. Lett. 1997, 30, 1-10.

85. Galal, A.; Wang, Z.; Karagozler, A. E.; Zimmer, H.; Mark, H. B.; Bishop, P. L. A Potentiometric Iodide (and Other) Ion Sensor-Based on a Conducting Polymer Film Electrode .2. Effect of Electrode Conditioning and Regeneration Techniques. Anal. Chim. Acta 1994, 299, 145-163.

86. Alizadeh, N.; Mahmodian, M. A new dodecylsulfate ion-selective sensor based on electrochemically prepared polypyrrole and PVC. Electroanalysis 2000, 12, 509-512.

87. Hofmeister, F. On the understanding of the effects of salts, second report. On irregularities in the precipitating effect of salts and their relationship to their physical behavior. Arch. Exp. Pathol. Pharmacol. 1888, 24, 247-260.

88. Ganjali, M. R.; Moghaddamb, M. R.; Norouzi, P.; Shirvani-Arani, S.; Daneshgar, P.; Adib, M.; Sobhi, H. R. Highly selective and sensitive triiodide PVC-based membrane electrode based on a 
new charge transfer complex of 2-(((2-(((E)-1-(2-hydroxyphenyl) methylidine) amino) phenyl) imino) methyl) phenol for nano-level monitoring of triiodide. Anal. Lett. 2006, 39, 683-695.

89. Xu, D. F.; Katsu, T. O,O,O-trialkyl phosphorothioates as simple and effective ionophores for silver ion-selective membrane electrodes. Anal. Chim. Acta. 2001, 443, 235-240.

90. Saad, B.; Zin, Z. M.; Jab, M. S.; Rahman, I. A.; Saleh, M. I.; Mahsufi, S. Flow-through chloroquine sensor and its applications in pharmaceutical analysis. Anal. Sci. 2005, 21, 521-524.

91. Ganjali, M. R.; Kiani-Anbouhi, R.; Shamsipur, M.; Poursaberi, T.; Salavati-Niasari, M.; Talebpour, Z.; Emami, M. Novel potentiometric PVC-membrane and coated graphite sensors for lanthanum(III). Electroanalysis 2004, 16, 1002-1008.

92. Segui, M. J.; Lizondo-Sabater, J.; Benito, A.; Martinez-Manez, R.; Pardo, T.; Sancenon, F.; Soto, J. A new ion-selective electrode for anionic surfactants. Talanta 2007, 71, 333-338.

93. Segui, M. J.; Lizondo-Sabater, J.; Martinez-Manez, R.; Sancenon, F. ; Soto, J. ; Garcia-Breijo, E.; Gil, L. An ion-selective electrode for anion perchlorate in thick-film technology. Sensors 2006, 6, 480-491.

94. Siswata, D.; Hisamoto, H.; Tohma, H.; Yamamoto, N.; Suzuki, K. Novel ammonium ionophores based on glycol dibenzy ethers for an Ion-Selective Eelectrode. Chem. Lett. 1994, 5, 945-948.

95. Shamsipur, M.; Hosseini, M.; Alizadeh, K.; Talebpour, Z.; Mousavi, M.F.; Ganjali, M.R.; Arca, M.; Lippolis, V. PVC membrane and coated graphite potentiometric sensors based on Et(4)todit for selective determination of samarium(III). Anal. Chem. 2003, 75, 5680-5986.

96. Akl, M. A.; Ghoneim, A. K.; Abd El-Aziz, M. H. Novel plastic chromium (III)-ion selective electrodes based on different ionophoric species and plasticizing solvent mediators. Electroanalysis 2006, 18, 299-306.

98. Peper, S.; Gonczy, C.; Runde, W. $\mathrm{Cs}^{+}$-selective membrane electrodes based on ethylene glycolfunctionalized polymeric microspheres. Talanta 2005, 67, 713-717.

99. Zamani, H. A.; Abedini-Torghabeh, J.; Ganjali, M. R. A highly selective and sensitive calcium(II)-selective PVC membrane based on dimethyl 1-(4-nitrobenzoyl)-8-oxo-2,8-dihydro1H-pyrazolo[5,1-a]isoindole-2,3-dicarboxylate as a novel ionophore. Bull. Korean Chem. Soc. 2006, 27, 835-840.

100. Ganjali, M.R.; Emami, M.; Salavati-Niasari, M.; Yousefi, M. Determination of trace amounts of $\mathrm{Cr}(\mathrm{III})$ in presence of $\mathrm{Cr}(\mathrm{VI})$ by a novel potentiometric membrane sensor based on a new tridentate S,N,O Schiff's base. Anal. Lett. 2003, 36, 2735-2747.

101. Gupta, V. K.; Jain, A. K.; Ishtaiwi, Z.; Lang, H.; Maheshwari, G. Ni ${ }^{2+}$ selective sensors based on meso-tetrakis-\{4-[tris-(4-allyl dimethylsilyl-phenyl)-silyl]-phenyl $\}$ porphyrin and (sal)(2)trien in poly(vinyl chloride) matrix. Talanta 2007, 73, 803-811.

102. Ganjali, M. R.; Akbar, V.; Norouzi, P.; Moghimi, A. Sepehrifard A. Be(II) graphite coated membrane sensor based on a recently synthesized benzo-9-crown-3 derivative, Electroanalysis 2005, $17,895-900$.

103. Faridbod, F.; Ganjalli, M. R.; Larijani, B.; Norouzi, P.; Riahi, S.; Mirnaghi, F. S. Lanthanide Recognition: an Asymetric Erbium Microsensor Based on a Hydrazone Derivative. Sensors 2007, 7, 3119-3135. 
104. Katsu, T.; Ido, K.; Kataoka, K. Poly(vinyl chloride) membrane electrode for a stimulant, phentermine, using tris(2-ethylhexyl) phosphate as a solvent mediator. Sens. Actuators B 2002, 81, 267-272.

105. Ganjali, M. R.; Emami, M.; Rezapour, M.; Shamsipur, M.; Maddah, B.; Salavati-Niasari, M.; Hosseini, M. Talebpour, Z. Novel gadolinium poly(vinyl chloride) membrane sensor based on a new S-N Schiff's base. Anal. Chim. Acta 2003, 495, 51-59.

106. Masadome, T.; Asano, Y. Determination of sulfate ion by potentiometric back-titration using sodium tetrakis (4-fluorophenyl) borate as a titrant and a titrant-sensitive electrode. Talanta 1999, 48, 669-673.

107. Ganjali, M. R.; Mizani, F.; Salavati-Niasari, M. Novel monohydrogenphosphate sensor based on vanadyl salophen. Anal. Chim. Acta 2003, 481, 85-90.

108. Walton J. Cabrera, Eduardo S. Maldonado, Hern’an E. R'1osy, Effect of Dodecyl Alcohol on the Potentiometric Response of an Isopropyl Xanthate Ion-Selective Electrode. J. Colloid Interface Sci. 2001, 237, 76-79.

109. Izatt, R. M.; Pawlak, K.; Bradshaw, J. S.; Bruening, R. L. Thermodynamic and kinetic data for macrocycle interaction with cations and anions. Chem. Rev. 1991, 91, 1721.

110. Yoshio, M.; Noguchi, H. Crown ethers for chemical analysis a review. Anal. Lett. 1982, 15, 1197-1276.

111. Takagi, M.; Nakamura, H. Analytical application of functionalized crown-ether metalcomplexes. J. Coord.Chem. 1986, 15, 53-82.

112. Izatt, R. M.; Clark, G. A.; Bradshaw, J. S.; Lamb, J. D.; Christensen, J. J. Sep. Purif. Methods, 1986, 15, 21.

113. Mi,Y. M.; Bakker, F. Lipophilic ionic sites for solvent polymeric membrane $\mathrm{pH}$ electrodes based on 4',5'-dibromofluorescein octadecylester as electrically charged carrier, Electrochem. Soc. 1997, 27, 144.

114. Han, W. S.; Park, M. Y.; Chung, K. C.; Cho, D. H.; Hong, T. K. Enhanced electrochemical performance of poly(aniline) solid-contact $\mathrm{pH}$ electrodes based on alkyldibenzylamine, Anal. Sci. 2000, 16, 1145.

115. Abakumova, R. A.; Valiotti, A. B.; Vasil'eva, O. E.; Kopylova, E. A.; Shumilova,G. I.; Mikhel'son, K. N. pH sensitivity of a porphyrin film electrode. Russ. J. Gen. Chem. 2003, 73, 300.

116. Issa, T. B.; Singh, P.; Baker, M.; Verma, B. S. 1,1'-Bis(11-mercaptoundecyl)ferrocene for potentiometric sensing of $\mathrm{H}^{+}$ion in sulfuric acid media simulating lead acid battery electrolyte. $J$. Appl. Electrochem. 2001, 31, 921.

117. Han, W. S.; Yoo, S. J.; Kim, S. H.; Hong, T. K.; Chung, K. C. Behavior of a polypyrrole solid contact $\mathrm{pH}$-selective electrode based on tertiary amine ionophores containing different alkyl chain lengths between nitrogen and a phenyl group. Anal. Sci. 2003, 19, 357.

118. Han, W. S.; Park, M. Y.; Cho, D. H.; Hong, T. K.; Lee, D. H.; Park, J. M.; Chung, K. C. The behavior of a poly(aniline) solid contact $\mathrm{pH}$ selective electrode based on N,N,N',N 'tetrabenzylethanediamine ionophore. 2001, Anal. Sci. 17, 727. 
119. Demirel, A.; Dogan, A.; Canel, E.; Memon, S.; Yilmaz, M.; Kilic, E. Hydrogen ion-selective poly(vinyl chloride) membrane electrode based on a p-tert-butylcalix[4]arene-oxacrown-4. Talanta 2004, 62, 123.

120. Han,W. S.; Park, M. Y.; Chung, K.; Cho, D. H.; Hong, T. K. Potentiometric sensor for hydrogen ion based on $\mathrm{N}, \mathrm{N}$ '-dialkylbenzylethylenediamine neutral carrier in a poly(vinyl chloride) membrane with polyaniline solid contact. Talanta, 2001, 54, 153.

121. Martelli, P. B.; Reis, B. F.; Zagatto, E. A.G.; Lima, J. L. F. C.; Lapa, R. A. Construction and evaluation of a tubular ion-selective electrode for hydrogen ion as a detector in flow analysis. Quimica 1998, 21, 133.

122. Heng, L. Y.; Chern, L. H.; Ahmad, M. A hydrogen ion-selective sensor based on non-plasticised methacrylic-acrylic membranes. Sensors 2002, 2, 339.

123. Cho, D. H.; Chung, K. C.; Park, M. Y. Hydrogen ion-selective membrane electrodes based on alkyldibenzylamines as neutral carriers. Talanta 1998, 47, 815.

124. Han, W. S.; Park, M. Y.; Chung, K. C.; Cho, D. H.; Hong, T. K. All solid state hydrogen ion selective electrode based on a tribenzylamine neutral carrier in a poly(vinyl chloride) membrane with a poly(aniline) solid contact. Electroanalysis 2001, 13, 955.

125. Cretin, M.; Fabry, P. Detection and selectivity properties of $\mathrm{Li}^{+}$-ion-selective electrodes based on NASICON-type ceramics. Anal. Chim. Acta 1997, 354, 291.

126. Cretin, M.; Alerm, L.; Bartroli, J.; Fabry, P. Lithium determination in artificial serum using flow injection systems with a selective solid-state tubular electrode based on NASICON membranes. Anal. Chim. Acta 1997, 350, 7.

127. Kang, Y. R.; Lee, K. M.; Nam, H.; Cha, G. S.; Jung, S. O.; Kim, J. S. Lithium ion-selective electrodes employing tetrahydrofuran-based 16-crown-4 derivatives as neutral carriers. Analyst 1997, 122, 1445.

128. Sim, J. H.; Lee, K. M.; Lee, J. S.; Cho, D. H.; Nam, H.; Cha, G. S. Lithium ion-selective electrode with improved lifetime. Bull. Korean Chem. Soc. 2001, 22, 765.

129. Kim, Y. D.; Jeong, H.; Kang, S. O.; Nam, K. C.; Jeon, S. Polymeric membrane sodium ionselective electrodes based on calix[4]arene triesters. Bull. Korean Chem. Soc. 2001, 22, 405-408.

130. Abbas, M. N. A novel PVC membrane selective electrode for the determination of sodium nitroprusside in pharmaceutical preparations. Anal. Lett. 2002, 35, 813.

131. Tavakkoli, N. Sodium ion-selective membrane electrode based on dibenzopyridino-18-crown-6. Bull. Korean Chem. Soc. 2004, 25, 1474.

132. Chandra, S.; Lang, H. A new sodium ion selective electrode based on a novel silacrown ether. Sens. Actuators B, 2006, 114, 849.

133. Hassan, S. S. M.; Mahmoud, W. H.; Othman, A. H. M. A novel potassium ion membrane sensor based on rifamycin neutral ionophore Talanta, 1997, 44, 1087-1094.

134. Yamauchi, A. Hayashita, T. Nishizawa, S. Watanabe, M.; Teramae, N. Benzo-15-crown-5 fluoroionophore/gamma-cyclodextrin complex with remarkably high potassium ion sensitivity and selectivity in water. J. Am. Chem. Soc. 1999, 121, 2319-2320.

135. Quagraine, E. K.; Gadzekpo, V. P. Y. Studies of potassium ion-selective electrodes based on salicylaldoxime. Discovery Innovation 1998, 10, 41-45. 
136. Yamauchi, A.; Hayashita, T.; Kato, A.; Nishizawa, S.; Watanabe, M.; Teramae, N. Selective potassium ion recognition by benzo-15-crown-5 fluoroionophore/y-cyclodextrin complex sensors in water. Anal. Chem. 2000, 72, 5841-5846.

137. Pandey P. C.; Prakash, R. Polyindole modified potassium ion sensor using dibenzo-18-crown-6 mediated PVC matrix membrane. Sens. Actuators B 1998, 46, 61-65.

138. Hopartean, E.; Pica, E. M.; Ana, C.; Cosma, V.; Hopartean, I. Membrane based on decyl-18crown-6 for a potassium selective sensor. Chem. Anal. (Warsaw) 2001, 46, 41-49.

139. Heng, L. Y.; Hall, E. A. H. One-step synthesis of $\mathrm{K}^{+}$-selective methacrylic-acrylic copolymers containing grafted ionophore and requiring no plasticizer. Electroanalysis 2000, 12, 178-186.

140. Ganjali, M. R.; Moghimi, A.; Buchanan, G. W.; Shamsipur, M. The synthesis of styrene/4'-vinylbenzo-24-crown-8 copolymer and its use in potassium-ion selective electrodes. J. Inclus. Phenom. Mol. Rec. Chem. 1998, 30, 29-43.

141. Cha, T. D.; Chung, S. K.; Park, J.; Kim, H. Anal. Sci. 1997, 13, 325.

142. Alexander, P. W.; Dimitrakopoulos, T.; Hibbert, D. B. A photo-cured coated wire potassium ionselective electrode for use in flow injection potentiometry Electroanalysis 1997, 9, 813-817.

143. Oh, K. C.; Kang, E. C.; Cho, Y. L.; Jeong, K. S.; Yoo, E. A.; Peang, K. J. Potassium-selective PVC membrane electrodes based on newly synthesized cis- and trans-bis(crown ether)s. Anal. Sci. 1998, 14, 1009-1012.

144. Michalska, A. J.; Appaih-Kusi, C.; Heng, L. Y.; Walkiewicz, S.; Hall, E. A. H. An experimental study of membrane materials and inner contacting layers for ion-selective $\mathrm{K}^{+}$electrodes with a stable response and good dynamic range. Anal. Chem. 2004, 76, 2031.

145. Xia, Z. R.; Badr, I. H. A.; Plummer, S. L.; Cullen, L.; Bachas, L. G. Synthesis and evaluation of a bis(crown ether) ionophore with a conformationally constrained bridge in ion-selective electrodes. Anal. Sci. 1998, 14, 169.

146. Hyun, M. H.; Piao, M. H.; Cho, Y. J.; Shim, Y. B. Ionophores in rubidium ion-selective membrane electrodes. Electroanalysis 2004, 16, 1785.

147. Kang, Y. R.; Oh, H. J.; Lee, K. M.; Cha, G. S.; Nam, H.; Paek, K.; Ihm, H. Potentiometric characteristics of ion-selective electrodes based on upper-rim calix[4]crown neutral carrier. Bull. Korean Chem. Soc. 1998, 19, 207-211.

148. Kim, J. S.; Ohki, A.; Ueki, R.; Ishizuka, T.; Shimotashiro, T.; Maeda, S. Cesium-ion selective electrodes based on calix[4] arene dibenzocrown ethers Talanta 1999, 48, 705.

149. Oh, H.; Choi, E.M.; Jeong, H.; Nam, K.C.; Jeon, S. Poly(vinyl chloride) membrane cesium ionselective electrodes based on lipophilic calix[6]arene tetraester derivatives Talanta, 2000, 53, 535-542.

150. Ashassi-Sorkhabi, H.; Rostamikia, T.; Shahrisa, A.; Banaei, A. Cesium-ion selective electrode based on pyrone compound. Bull. Electrochem. 2001, 17, 545-548.

151. Choi, E. M.; Oh, H.; Ko, S. W.; Choi, Y. K.; Nam, K. C.; Jeon, S. Polymeric membrane cesiumselective electrodes based on quadruply-bridged calix[6]arenas. Bull. Korean Chem. Soc. 2001, $22,1345-1349$.

152. Chen, L. X.; Ju, H. F.; Zeng, X. S.; He, X. W.; Zhang, Z. Z. Cesium selective electrodes based on novel double flexible spacers bridged biscalix[4]arenas. Anal. Chim. Acta, 2001, 447, 41-46. 
153. Arvand-Barmchi, M.; Mousavi, M. F.; Zanjanchi, M. A.; Taghvaei, S. Cesium-selective poly(vinylchloride) membrane electrode based on a new calix[4]arene derivative in the 1,3alternate conformation. Anal. Lett. 2002, 35, 767-783.

154. Arvand-Barmchi, M.; Mousavi, M. F.; Zanjanchi, M. A.; Shamsipur, M. A PTEV-based zeolite membrane potentiometric sensor for cesium ion. Sens. Actuators B 2003, 96, 560-564.

155. Ganjali, M. R.; Nourouzi, P.; Emami, M.; Golmohammadi, M.; Moradzadegun, A. Novel Cesium membrane sensor based on a cavitand. J. Chin. Chem. Soc. 2006, 53, 1209-1214.

156. Arida, H. A. M.; Aglan, R. F.; El-Reefy, S.A. A new cesium ion selective graphite rod electrode based on Cs-molybdophosphate. Anal. Lett. 2004, 37, 21-33.

157. Peper, S.; Gonczy, C.; Runde, W. $\mathrm{Cs}^{+}$-selective membrane electrodes based on ethylene glycolfunctionalized polymeric microspheres. Talanta 2005, 67, 713-717.

158. Ganjali, M. R.; Moghimi, A.; Shamsipur, M. Beryllium-selective membrane electrode based on benzo-9-crown-3. Anal. Chem. 1998, 70, 5259-5263.

159. Ganjali, M. R.; Daftari, A.; Rastegar, M.F.; Moghimi, A. Novel beryllium membrane sensor based on 2,4-dinitrophenylhydrazinebenzo-9-crown-3 coated graphite. Anal. Lett. 2003, 36, 317-328.

160. Ganjali, M. R.; Daftari, A.; Faal-Rastegar, M.; Moghimi, A. Novel potentiometric sensor for monitoring beryllium based on naphto-9-crown-3. Anal. Sci. 2003, 19, 353-356.

161. Ganjali, M. R.; Ghorbani, M.; Norouzi, P.; Daftari, A.; Faal-Rastegar, M.; Moghimi, A. Nano levels detection of beryllium by a novel beryllium PVC-based membrane sensor based on 2,3,5,6,8,9-hexahydro-1,4,7,10-benzotetraoxacyclododecine-12-carbaldehyde-12-(2,4-dinitrophenyl) hy. Sens. Actuators B 2004, 100, 315-319.

162. Ganjali, M. R.; Rahimi-Nasrabadi, M.; Maddah, B.; Moghimi, A.; Faal-Rastegar, M.; Borhany, S.; Namazian, M. Sub-micro level monitoring of Beryllium ions with a novel beryllium sensor based on 2,6-diphenyl-4-benzo-9-crown-3- pyridine. Talanta 2004, 63, 899-906.

163. Ganjali, M. R.; Norouzi, P.; Faridbod, F.; Sepehrifard, A.; Ghandi, M.; Moghimi, A. Monitoring of beryllium(II) ions by use of a novel beryllium(II) membrane sensor, based on 2, 3, 5, 6, 8, 9hexahydronaphto [2,3-b][1, 4, 7, 10] tetraoxacyclo dodecine. Can. J. Anal. Sci. Spect. 2007, 52, 46-56.

164. Ganjali, M. R.; Daftari, A.; Pourjavid, M. R.; Rastegar, M. F.; Moghimi, A. Novel Be(II)membrane electrode-based on a derivative of benzo-9-crown-3. Main group Met. Chem. 2002, 25, 669-676.

165. Shamsipur, M.; Ganjali, M. R.; Rouhollahi, A.; Moghimi, A. Beryllium-selective membrane sensor based on 3,4-di[2-(2-tetrahydro-2H-pyranoxy)]ethoxy styrene-styrene copolymer. Anal. Chim. Acta 2001, 434, 23-27.

166. Zhang, X. J.; Fakler, A.; Spichiger, U. E. Development of magnesium-ion-selective microelectrodes based on a new neutral carrier ETHT 5504. Electroanalysis 1998, 10, 11741181.

167. Gupta, A. P.; Ikram, S.; Agarwal, H. Studies on zirconium(IV) selenomolybdate gel based $\operatorname{Mg}($ II) ion selective heterogeneous membrane sensor - Determination of water hardness. J. Sci. Ind. Res. 2002, 61, 61-66. 
168. Gupta, V. K.; Chandra, S.; Mangla, R. Magnesium-selective electrodes. Sens. Actuatorsors B, 2002, 86, 235-241.

169. van Staden, J. F.; Stefan, R.I. Simultaneous flow injection determination of calcium and fluoride in natural and borehole water with conventional ion-selective electrodes in series. Talanta 1999, 49, 1017-1022.

170. Lindfors, T.; Ivaska, A. Calcium-selective electrode based on polyaniline functionalized with bis[4-(1,1,3,3-tetramethylbutyl)phenyl]phosphate. Anal. Chim. Acta 2001, 437, 171-182.

171. Mahmoud, W. H. Novel bilirubin-based PVC calcium sensors. Anal. Sci. 2003, 19, 361-365.

172. Kumar, A.; Mittal, S. K. PVC based dibenzo-18-crown-6 electrode for Ca(II) ions. Sens. Actuators B 2004, 99, 340-343.

173. Ganjali, M. R.; Zamani, H. A.; Norouzi, P.; Adib, M.; Accedy, M. Novel Calcium sensor based on [2-(2-hydroxyphenyl)imino]-1,2-diphenylethanone. Acta Chim. Slov. 2005, 52, 309-316.

174. Bedlechowicz-Sliwakowska, I.; Lingenfelter, P.; Sokalski, T.; Lewenstam, A.; Maj-Zurawska, M. Ion-selective electrode for measuring low $\mathrm{Ca}^{2+}$ concentrations in the presence of high $\mathrm{K}^{+}, \mathrm{Na}^{+}$ and $\mathrm{Mg}^{2+}$ background. Anal. Bioanal. Chem. 2006, 385, 1477-1482.

175. Singh, A. K.; Mehtab, S. Calcium(II)-selective potentiometric sensor based on at-furildioxime as neutral carrier Sens. Actuators B, 2007, 123, 429-436.

174. Qian, G. Y.; Wu, M. B.; Wu, G. L.; Huang, S.; Yan, Y. K.; Tian, B. Z. Strontium ion-selective electrodes based on the diamides with pyridine ring as ionophores Talanta, 1998, 47, 1149-1155.

175. Gupta, V. K.; Jain, A. K.; Khurana, U.; Singh, L. P. PVC-based neutral carrier and organic exchanger membranes as sensors for the determination of $\mathrm{Ba}^{2+}$ and $\mathrm{Sr}^{2+}$. Sens. Actuators B, 1999, 55, 201-211.

176. Gupta, V. K.; Ludwig, R.; Agarwal, S. Strontium(II) sensor based on a modified calix[6]arene in PVC matrix. Anal. Sci. 2005, 21, 293-296.

177. Singh, A. K.; Saxena, P.; Mehtab, S.; Gupta, B. Strontium(II)-selective electrode based on a macrocyclic tetraamide. Talanta 2006, 69, 521-526.

178. Shamsipur, M.; Kazemi, S. Y. Design of a selective and sensitive PVC-membrane potentiometric sensor for strontium ion based on 1,10-diaza-5,6-benzo-4,7-dioxacyclohexadecane-2,9-dione as a neutral ionophore. Sensors 2007, 7, 438-447.

179. Zamani, H. A.; Ganjali, M. R.; Norouzi, P.; Adib, M. Strontium PVC-Membrane Sensor Based on 2-[(2-mercaptophenylimino) methyl]phenol. Material Sci. Eng.C 2008, 28, 157-163.

180. Ganjali, M. R.; Kiani, R.; Yousefi, M.; Faal-Rastegar, M. Novel potentiometric strontium membrane sensor based on dibenzo-30-crown-10. Anal. Lett. 2003, 36, 2123-2137.

181. Shamsipur, M.; Rouhani, S.; Shaghi, H.; Ganjali, M. R.; Eshghi, H. Strontium-selective membrane electrodes based on some recently synthesized benzo-substituted macrocyclic diamides. Anal. Chem. 1999, 71, 4938-4943.

182. Saleh, M. B. Neutral bidentate organophosphorus compounds as novel ionophores for potentiometric membrane sensors far barium(II). Fresenius J. Anal. Chem. 2000, 367, 530-534.

183. Hassan, S. S. M.; Saleh, M. B.; Gaber, A. A. A.; Kream, N. A. A. DDB liver drug as a novel ionophore for potentiometric barium(II) membrane sensor. Talanta 2003, 59, 161-166. 
184. Zamani, H. A.; Abedini-Torghabeh, J.; Ganjali, M. R. A highly selective and sensitive barium(II)-selective PVC membrane based on dimethyl 1-acetyl-8-oxo-2,8-dihydro-1hpyrazolo[5,1-a]isoindole-2,3-dicarboxylate. Electroanalysis 2006, 18, 888-893.

185. Mousavi, M. F.; Arvand-Barmchi, M.; Zanjanchi, M. A. Al(III)-Selective electrode based on furil as neutral carrier. Electroanalysis 2001, 13, 1125-1128.

186. Abbaspour, A.; Esmaeilbeig, A. R.; Jarrahpour, A. A.; Khajeh, B.; Kia, R. Aluminium(III)selective electrode based on a newly synthesized tetradentate Schiff base. Talanta 2002, 58, 397403.

187. Shamsipur, M.; Ershad, S.; Yari, A.; Sharghi, H.; Salimi, A. R. Hydroxy-thioxanthones as suitable neutral ionophores for the preparation of PVC-membrane potentiometric sensors for Al(III) ion. Anal. Sci. 2004, 20, 301-306.

188. Yari, A.; Darvishi, L.; and Shamsipur, M. Al (III)-selective electrode based on newly synthesized xanthone derivative as neutral ionophore. Anal. Chim. Acta 2006, 555, 329-335.

189. Gholivand, M. B.; Ahmadi, F.; Rafiee, E. A novel Al(III)-selective electrochemical sensor based on N,N '-bis(salicylidene)-1,2-phenylenediamine complexes. Electroanalysis 2006, 18, 16201626.

190. Yoshikuni, N.; Takeno, H.; Oguma, K. Preparation of gallium ion-selective membrane electrode by use of anion-exchange resin and its application to chemical analysis of industrial materials. Bunseki Kagaku 2001, 50, 753-757.

191. Mohamed, S. K. Ion-selective electrode for gallium determination in nickel alloy, fly-ash and biological samples. Anal. Chim. Acta 2006, 562, 204-209.

192. Li, C. X.; Hou, J. G. Construction and evaluation of a novel In(III) liquid ion-selective membrane electrode. Talanta 1997, 44, 729-732.

193. Abdulla, N. I.; Al-Haider, A. M.; Al-Joboury.; M. I.; Nassory, N. S. Construction and characterization of indium liquid ion selective electrodes based on crown ethers in a PVC matrix membrane. Turk. J. Chem. 2005, 29, 687-696.

194. Saleh, M. B. A novel PVC membrane sensor for potentiometric determination of thallium(I). $J$. Electroanal. Chem. 1998, 448, 33-39.

195. Katsu, T.; Ido, K.; Takaishi, K.; Yokosu, H. Thallium(I)-selective membrane electrodes based on calix[6]arene or calix[5]arene derivatives. Sens. Actuators B 87, 2002, 331-335.

196. Ganjali, M. R.; Pourjavid, M. R.; Mouradzadegun, K.; Hosseini, M.; Mizani, F. Novel thallium(I)-selective membrane electrode based on a podal ligand. Bull. Korean Chem. Soc. 2003, 24, 1585-1589.

197. Gaber, A. A. A. New thallium(I) ion selective electrode based on indeno pyran compound. Sens. Actuators B 2003, 96, 615-620.

198 Khayatian, G.; Shariati, S.; Salimi, A. Thallium(I)-selective membrane potentiometric sensor based on dibenzyldiaza-18-crown-6. Bull. Korean Chem. Soc. 2003, 24, 421-425.

199. Hassanien, M. M.; Abou-El-Sherbini, K. S.; Mostafa, G. A. E. A novel tetrachlorothallate (III)PVC membrane sensor for the potentiometric determination of thallium (III). Talanta 2003, 59, 383-392. 
200. Kharitonov, S. V.; Zarembo, Y. V.; Zarembo, V. I. Novel thallium(III) solid-contact ion-selective electrode with electropolymerized transducer Electroanalysis 2006, 18, 1354-1362.

201. Aghaie, H.; Giahi, A.; Monajjemi, M.; Arvand, A.; Nafissi, G. H.; Aghaie, M. Tin(II)-selective membrane potentiometric sensor using a crown ether as neutral carrier. Sens. Actuators B $\mathbf{2 0 0 5}$, 107, 756-761.

202. Arvand, M.; Moghimi, A. M.; Afshari, A.; Mahmoodi, N. Tin(II)-selective membrane potentiometric sensor using a crown ether as neutral carrier. Anal. Chim. Acta, 2006, 579, 102108.

203. Casado, M.; Daunert, S.; Valiente, M. Lead-selective electrode based on a quinaldic acid derivative. Electroanalysis 2001, 13, 54-60.

204. Mousavi, M. F.; Barzegar, M. B.; Sahari, S. A PVC-based capric acid membrane potentiometric sensor for lead(II) ions. Sens. Actuators B 2001, 73, 199-204.

205. Sadeghi, S.; Dashti, G. R.; Shamsipur, M. Lead-selective poly(vinyl cholride) membrane electrode based on piroxicam as a neutral carrier. Sens. Actuators B 2002, 81, 223-228.

206. Gholivand, M. B.; Mohammadi, A. Lead ion-selective membrane electrode containing 2,2'dithiodibenzoic acid as ionophore. Chem. Anal. (Warsaw) 2003, 48, 305-312.

207. Riahi, S.; Mousavi, M. F.; Shamsipur, M.; Sharghi, H. A novel PVC-membrane-coated graphite sensor based on an anthraquinone derivative membrane for the determination of lead Electroanalysis 2003, 15, 1561-1565.

208. Bhat, V. S.; Ijeri, V. S.; Srivastava, A. K. Coated wire lead(II) selective potentiometric sensor based on 4-tert-butylcalix[6]arene. Sens. Actuators B, 2004, 99, 98-105.

209. Lee, H. K.; Song, K.; Seo, H. R.; Choi, Y. K.; Jeon, S. Lead(II)-selective electrodes based on tetrakis(2-hydroxy-1-naphthyl)porphyrins: the effect of atropisomers. Sens. Actuators B 2004, 99, 323-329.

210. Pouretedal, H. R.; Keshavarz, M. H. Lead (H)-selective electrode based on dibenzodiaza-15crown-4. Asian J. Chem. 2004, 16, 1319-1326.

211. Yan, Z. N.; Hou, Y. H.; Zhou, Z. X.; Du, C. X.; Wu, Y. J. Lead ion-selective membrane electrode based on a novel fluorene derivative. Anal. Lett. 2004, 37, 3149-3159.

212. Zare, H. R.; Ardakani, M. M.; Nasirizadeh, N.; Safari, J. Lead-selective poly(vinyl chloride) membrane electrode based on 1-phenyl-2-(2-quinolyl)-1,2-dioxo-2-(4-bromo) phenylhydrazone. Bull. Korean Chem. Soc. 2005, 26, 51-56.

213. Ardakani, M. M.; Zare, H. R.; Nasirizadeh, N.; Safari, J. Highly selective lead (II) membrane electrode based on new oxim phenyl 2-keto methyl quinoline (OPKMQ). Can. J. Anal. Sci. Spect. 2004, 49, 226-233.

214. Perez-Marin, L.; Macedo-Miranda, G.; Avila-Perez, P.; Otazo-Sanchez, E.; Carrasco-Abrego, H.; Lopez-Valdivia, H.; Alonso-Chamarro, J.; Estevez-Hernandez, O. Study of 1-furoil-3(2hydroxiethyl)thiourea as ionophore for lead (II) in electrode of liquid membrane of neutral carrier. Afinidad 1999, 56, 295-298.

215. Xu, D. F.; Katsu, T. Lead-selective membrane electrode based on dibenzyl phosphate. Anal. Chim. Acta 1999, 401, 111-115. 
216. Xu, D. F.; Katsu, T. Tetrabenzyl pyrophosphate as a new class of neutral carrier responsive to lead ion. Talanta 2000, 51, 365-371.

217. Sadeghi, S.; Shamsipur, M. Lead(II)-selective membrane electrode based on tetraphenylporphyrin. Anal. Lett. 2000, 33, 17-28.

218. Marin, L. P.; Rubi, Y. M.; de Fuentes, O. A.; Ortiz, O. F.; Sanchez, E. O.; Estevez, O. L.; Fajardo, Y.; Chamorro, J. A.; Casabo, J. Lead ion-selective electrode based on a neutral mobile carrier. Afinidad 1998, 55, 130-132.

219. Yang, X. H.; Kumar, N.; Hibbert, D. B.; Alexander, P. W. Lead(II)-selective membrane electrodes based on 4,7,13,16-tetrathenoyl-1,10-dioxa-4,7,13,16-tetraazacyclooctadecane Electroanalysis 1998, 10, 827-831.

220. Ganjali, M. R.; Roubollahi, A.; Mardan, A. R.; Hamzeloo, M.; Mogimi, A.; Shamsipur, M. Lead ion-selective electrode based on 4'-vinylbenzo-15-crown-5 homopolymer. Microchem. J. 1998, 60, 122-133.

221. Ohki, A.; Kim, J. S.; Suzuki, Y.; Hayashita, T.; Maeda, S. Lead-selective poly(vinyl chloride) membrane electrodes based on acyclic dibenzopolyether diamides. Talanta 1997, 44, 1131-1135.

222. Yang, X. H.; Kumar, N.; Chi, H.; Hibbert, D. B.; Alexander, P. W. Lead-selective membrane electrodes based on dithiophenediazacrown ether derivatives. Electroanalysis 1997, 9, 549-553.

223. Jeong, T.; Jeong, D. C.; Lee, H. K.; Jeon, S. Lead(II)-selective polymeric electrode using a Schiff base complex of N,N '-bis-thiophene-2-ylmethylene-ethane-1,2-diamine as an ion carrier. Bull. Korean Chem. Soc. 2005, 26, 1219-1224.

224. Chen, L. X.; Zhang, J.; Zhao, W. F, He, X. W.; Liu, Y. Double-armed calix[4]arene amide derivatives as ionophores for lead ion-selective electrodes. J. Electroanal. Chem. 2006, 589, 106111.

225. Tavakkoli, N.; Khojasteh, Z.; Sharghi H, Shamsipur, M. Lead ion-selective membrane electrodes based on some recently synthesized 9,10-anthraquinone derivatives. Anal. Chim. Acta 1998, 360, 203-208.

226. Rouhollahi, A.; Ganjali, M. R.; Shamsipur, M. Lead ion selective PVC membrane electrode based on 5,5'-dithiobis-(2-nitrobenzoic acid). Talanta 1998, 46, 1341-1346.

227. Pouretedal, H. R.; Forghaniha, A.; Sharghi, H.; Shamsipur, M. Lead-selective membrane potentiometric sensor based on a recently synthesized bis(anthraquinone) sulfide derivative. Anal. Lett. 1998, 31, 2591-2606.

228. Lazo, A. R.; Bustamante, M.; Arada, M. A.; Jimenez, J.; Yazdani-Pedram, M. Construction and characterization of a lead(II) ion selective electrode with 1-furoil-3,3-diethylthiourea as neutral carrier. Afinidad 2005, 62, 605-610.

229. Zhang, W. J.; Li, C. Y.; Zhang, X. B.; Jin, Z. Synthesis of an amide-linked diporphyrin xanthene as a neutral carrier for a lead(II)-sensitive electrode. Anal. Lett. 2007, 40, 1023-1035.

230. Arida, H. A.; El-Reefy, S. A.; El-Saied, A. M. A new lead(II)-selective PVC-coated graphite rod electrode based on a Schiff base complex. Anal. Sci. 2003, 19, 687-690.

231. Ardakany, M. M.; Ensafi, A. A.; Naeimi, H.; Dastanpour, A.; Shamlli, A. Highly selective lead(II) coated-wire electrode based on a new Schiff base. Sens. Actuators B, 2003, 96, 441-445. 
232. Ardakany, M. M.; Ensafi, A. A.; Naeimi, H.; Dastanpour, A.; Shamlli, A. Coated, wire-based, new Schiff base potentiometric sensor for lead(II) ion. Russ. J. Electrochem. 2003, 39, 269-273.

233. Ardakani, M. M.; Kashani, M. K.; Salavati-Niasari, M.; Ensafi, A. A. Lead ion-selective electrode prepared by sol-gel and PVC membrane techniques. Sens. Actuators B, 2005, 107, 438445.

234. Jeong, T.; Lee, H. K.; Jeong, D. C.; Jeon, S. A lead (II)-selective PVC membrane based on a Schiff base complex of N,N'-bis(salicylidene)-2,6-pyridinediamine. Talanta 2005, 65, 543-548.

235. Ardakani, M. M.; Pourhakak, P.; Salavati-Niasari, M. Bis(2-hydroxyacetophenone)ethylenediimine as a neutral carrier in a coated-wire membrane electrode for lead(II). Anal. Sci. 2006, 22, 865-870.

236. Gupta, V. K.; Jain, A. K.; Kumar, P. PVC-based membranes of N,N '-dibenzyl-1,4,10,13tetraoxa-7,16-diazacyclooctadecane as $\mathrm{Pb}(\mathrm{II})$-selective sensor. Sens. Actuators B, 2006, 120, 259265.

237. Fathi, M. R.; Darviche, F.; Ganjali, M. R. Lead-selective membrane potentiometric sensor based on a recently synthesized dimethyl benzo tetrathia fulvalene. Anal. Lett. 2000, 33, 1025-1035.

238. Shamsipur, M.; Ganjali, M. R.; Rouhollahi, A. Lead-selective membrane potentiometric sensor based on an 18-membered thiacrown derivative. Anal. Sci. 2001, 17, 935-938.

239. Ganjali, M. R.; Hosseini, M.; Basiripour, F.; Javanbakht, M.; Hashemi, O. R.; Rastegar, M. F.; Shamsipur, M.; Buchanen, G. W. Novel coated-graphite membrane sensor based on N,N 'dimethylcyanodiaza-18-crown-6 for the determination of ultra-trace amounts of lead. Anal. Chim. Acta 2002, 464, 181-186.

240. Ganjali, M. R.; Daftari, A.; Norouzi, P.; Salavati-Niasari, M. Novel Y(III) PVC-based membrane microelectrode based on a new S-N Schiff's base. Anal. Lett. 2003, 36, 1511-1522.

241. Gholivand, M. B.; Babakhanian, A.; Joshaghani, A. Zirconium ion selective electrode based on bis(diphenylphosphino) ferrocene incorporated in a poly(vinyl chloride) matrix. Anal. Chim. Acta 2007, 584, 302-307.

242. Ganjali, M. R.; Norouzi, P.; Faridbod, F.; Riahi, S.; Ravanshad, J.; Tashkhourian, J.; SalavatiNiasari, M.; Javaheri M. Determination of Vanadyl Ions by a New PVC Membrane Sensor Based on N, N'-bis-(Salicylidene)-2,2-Dimethylpropane-1,3-Diamine. IEEE Sensors J. 2007, 7, 544550.

243. Ganjali, M. R.; Mizani, F.; Salavati-Niasari, M.; Javanbakht M. Novel potentiometric membrane sensor for the determination of trace amounts of chromium(III) ions. Anal. Sci. 2003, 19, 235 238.

244. Shamsipur, M.; Soleymanpour, A.; Akhond, M.; Sharghi, H.; Sarvari, M. H. Highly selective chromium(III) PVC-membrane electrodes based on some recently synthesized Schiff's bases. Electroanalysis 2005, 17, 776-772.

245. Ganjali, M.R.; Norouzi, P.; Faridbod, F.; Ghorbani, M.; Adib, M. Highly selective and sensitive chromium(III) membrane sensors based on a new tridentate Schiff's base. Anal. Chim. Acta 2006, $569,35-41$. 
246. Akl, M. A.; Ghoneim, A. K.; Abd El-Aziz, M. H. Novel plastic chromium (III)-ion selective electrodes based on different ionophoric species and plasticizing solvent mediators Electroanalysis 2006, 18, 299-306.

247. Gupta, V. K.; Jain, A. K.; Kumar, P.; Agarwal, S.; Maheshwari, G. Chromium(III)-selective sensor based on tri-o-thymotide in PVC matrix. Sens. Actuators B, 2006, 113, 182-186.

248. Gholivand, M. B.; Raheedayat, F. Chromium(III) ion selective electrode based on oxalic acid bis(cyclohexylidene hydrazide). Electroanalysis 2004, 16, 1330-1335.

249. Khalil, S.; Wassel, A. A.; Belal, F. F. Coated graphite-epoxy ion-selective electrode for the determination of chromium(III) in oxalic medium. Talanta 2004, 63, 303-307

250. Sil, A.; Ijeri, V. S.; Srivastava, A. K. Coated wire chromium(III) ion-selective electrode based on azamacrocycles. Anal. Bioanal. Chem. 2004, 378, 1666-1669.

251. Abbaspour, A.; Izadyar, A. Chromium(III) ion-selective electrode based on 4dimethylaminoazobenzene. Talanta 2001, 53, 1009-1013.

252. Zamani, H. A.; Rajabzadeh, G.; Ganjali, M. R. Highly selective and sensitive chromium(III) membrane sensors based on 4-amino-3-hydrazino-6-methyl-1,2,4-triazin-5-one as a new neutral ionophore. Sens. Actuators B 2006, 119, 41-46.

253. Abbaspour, A.; Izadyar, A. Carbon nanotube composite coated platinum electrode for detection of $\mathrm{Cr}(\mathrm{III})$ in real samples. Talanta 2007, 71, 887-892.

254. Zamania, H. A.; Ganjali, M. R.; Abedi, M. R.; Norouzi, P. Cr(III) Ion-Selective Membrane Sensor Based on 1,3-Diamino-2-hydroxypropane-N,N,N',N'-tetraacetic acid. Sens. Lett. 2007, 5, 516-521.

255. Gupta, V. K.; Jain, A. K.; Maheshwari, G. Manganese (II) selective PVC based membrane sensor using a Schiff base. Talanta 2007, 72, 49-53.

256. Singh, A. K.; Saxena, P.; Panwar, A. Manganese(II)-selective PVC membrane electrode based on a pentaazamacrocyclic manganese complex. Sens. Actuators B, 2005, 110, 377-381.

257. Mashhadizadeh, M. H.; Shoaei, I. S.; Monadi, N. A novel ion selective membrane potentiometric sensor for direct determination of Fe(III) in the presence of Fe(II). Talanta 2004, 64, 1048-1052.

258. Gupta, V. K.; Jain, A. K.; Agarwal, S.; Maheshwari, G. An iron(III) ion-selective sensor based on a mu-bis(tridentate) ligand. Talanta 2007, 71, 1964-1968.

259. Ekmekci, G.; Uzun, D.; Somer, G.; Kalayci, S. A novel iron(III) selective membrane electrode based on benzo-18-crown-6 crown ether and its applications. J. Membr. Sci. 2007, 288, 36-40.

260. Teixeira, M. F. D.; Pinto, A. Z.; Fatibello, O. Coated graphite-epoxy ion-selective electrode for the determination of iron(III) in oxalic medium. Anal. Lett. 1997, 30, 417-427.

261. Teixeira, M. F. D.; Aniceto, C.; Fatibello, O. Ion-selective electrode for the determination of iron(III) in vitamin formulations. JBCS 1998, 9, 506-510.

262. Saleh, M. B. Iron(III) ionophores based on formylsalicylic acid derivatives as sensors for ionselective electrodes. Analyst 1999, 125, 179-183.

263. Teixeira, M. F. D.; Fatibello, O.; Aniceto, C.; Neto, C. O. C. Flow-injection potentiometric determination of iron (III) in vitamin formulations using a tubular ion-selective electrode in oxalic medium. Lab. Rob. Autom. 1999, 11, 163-168. 
264. Sil, A.; Ijeri, V. S.; Srivastava, A. K. Coated-wire iron(III) ion-selective electrode based on iron complex of 1,4,8,11-tetraazacyclotetradecane. Sens. Actuators B, 2005, 106, 648-653.

265. Fakhari, A. R.; Alaghemand, M.; Shamsipur, M. Iron(III)-selective membrane potentiometric sensor based on 5,10,15,20-tetrakis(pentafluorophenyl)-21H, 23H-porphyrin. Anal. Lett. 2001, 34, 1097-1106.

266. Mahmoud, W. H. Iron ion-selective electrodes for direct potentiometry and potentiotitrimetry in pharmaceuticals. Anal. Chim. Acta 2001, 436, 199-206.

267. Zamania, H. A.; Rajabzadeha, G.; Pooyamanesh, M. J. ;Ganjali, M. R.; Norouzi, P. Fe(III) IonSelective Membrane Electrode Based on 4-Amino-6-methyl-3-methylmercapto-1,2,4-triazin-5one. Anal. Lett. 2007, 40, 1596-1609.

268. Mashhadizadeh, M. H.; Sheikhshoaie, I. $\mathrm{Co}^{2+}$-selective membrane electrode based on the Schiff base NADS. Anal. Bioanal. Chem. 2003, 375, 708-712.

269. Gupta, V. K.; Singh, A. K.; Mehtab, S.; Gupta, B. A cobalt(II)-selective PVC membrane based on a Schiff base complex of N,N '-bis(salicylidene)-3,4-diaminotoluene. Anal. Chim. Acta 2006, $566,5-10$.

270. Shamsipur, M.; Poursaberi, T.; Rouhani, S.; Niknam, K.; Sharghi, H.; Ganjali, M. R. Cobalt(II)selective membrane electrode based on a recently synthesized benzo-substituted macrocyclic diamide. Anal. Sci. 2001, 17, 1049-1054.

271. Singh, A. K.; Singh, R. P.; Saxena, P. Cobalt(II)-selective electrode based on a newly synthesized macrocyclic compound. Sens. Actuators B, 2006, 114, 578-583.

272. Shamsipur, M.; Rouhani, S.; Poursaberi, T.; Ganjali, M. R.; Sharghi, H.; Niknam, K. Cobalt(II)selective coated graphite PVC-membrane electrode based on a recently synthesized dibenzopyridino-substituted macrocyclic diamide. Electroanalysis 2002, 14, 729-735.

273. Ganjali, M. R.; Mizani, F.; Emami, M.; Darjezini, M.; Darvich, M. R.; Yousefi, M. Synthesis of a new oxime and its application to the construction of a highly selective and sensitive Co(II) PVC-based membrane sensor. Anal. Sci. 2004, 20, 531-535.

274. Mashhadizadeh, M. H.; Momeni, A.; Razavi, R. Cobalt(II)-selective membrane electrode using a recently synthesized mercapto compound. Anal. Chim. Acta 2002, 46, 245-252.

275. Shamsipur, M.; Rouhani, S.; Poursaberi, T.; Ganjali, M. R.; Sharghi, H.; Niknam, K. Cobalt(II)selective coated graphite PVC-membrane electrode based on a recently synthesized dibenzopyridino-substituted macrocyclic diamide. Electroanalysis 2002, 14, 729-735.

276. Ashtamkar, S. M.; Thakkar, N. V. Preparation and study of an epoxy resin based cobalt(II) ion selective electrode using a cobalt(II) complex. J. Indian Chem. Soc. 2002, 79, 90-91.

277. Shamsipur, M.; Poursaberi, T.; Rouhani, S.; Niknam, K.; Sharghi, H.; Ganjali, M. R. Cobalt(II)selective membrane electrode based on a recently synthesized benzo-substituted macrocyclic diamide. Anal. Sci. 2001, 17, 1049-1054.

278. Zamani, H. A.; Ganjali, M. R.; Norouzi, P.; Adib, M. Cobalt(II) Ion Detection in Electroplating Wastewater by a New Cobalt Ion-Selective Electrode Based on N'-[1-(2-thienyl)ethylidene]-2furohydrazide. J. cheilian Chem. Soc. 2007, 52, 1332-1337. 
279. Zamani, H. A.; Ganjali, M. R.; Norouzi, P.; Adib, M. Cobalt(II) ion detection in electroplating wastewater by a new cobalt ion-selective electrode based on N'-[1-(2-thienyl)ethylidene]-2furohydrazide. Sens. Lett. 2007, 5, 522-527.

280. Gupta, V. K.; Jain, A. K.; Singh, L. P.; Khurana, U. Porphyrins as carrier in PVC based membrane potentiometric sensors for nickel(II). Anal. Chim. Acta 1997, 355, 33-41.

281. Ganjali, M. R. Nickel(II) ion-selective electrode based on 2-methyl-4-(4-methoxy phenyl)-2,6diphenyl-2H-thiopyran. Electroanalysis 2000, 12, 1138-1142.

282. Jain, A. K.; Gupta, V. K.; Singh, R. D.; Khurana, U.; Singh, L. P. Nickel(II)-selective sensors based on heterogeneous membranes of macrocyclic compounds. Sens. Actuators B, 1997, 40, 1520.

283. Ganjali, M. R.; Fathi, M. R.; Rahmani, H.; Pirelahi, H. Nickel(II) ion-selective electrode based on 2,5-thiophenyl bis(5-tert-butyl-1,3-benzoxazole). Anal. Lett. 2000, 33, 3139-3152.

284. Belhamel, K.; Ludwig, R.; Benamor, M. Nickel ion-selective PVC membrane electrode based on a new t-octyl-calix[6]arene derivative. Microchim. Acta 2005, 149, 145-150.

285 Mashhadizadeh, M. H.; Sheikhshoaie, I.; Saeid-Nia, S. Nickel(II)-selective membrane potentiometric sensor using a recently synthesized Schiff base as neutral carrier. Sens. Actuators B 2003, 94, 241-246.

286. Gupta, V. K.; Prasad, R.; Kumar, A. Dibenzocyclamnickel(II) as ionophore in PVC-matrix for $\mathrm{Ni}^{2+}$-selective sensor. Sensors 2002, 2, 384-396.

287. Ganjali, M. R.; Hosseini, M.; Salavati-Niasari, M.; Poursaberi, T.; Shamsipur, M.; Javanbakht, M.; Hashemi, O. R. Nickel ion-selective coated graphite PVC-membrane electrode based on benzylbis(thiosemicarbazone). Electroanalysis 2002, 14, 526-531.

288. Mazloum, M.; Salavati-Niasari, M.; Amini, M. K. Nickel ion-selective coated graphite PVCmembrane electrode based on benzylbis(thiosemicarbazone). Sens. Actuators B 2002, 82, 259264.

289. Singh, A. K.; Sharma, C. L.; Baniwal, S.; Panwar, A. Nickel(II)-selective membrane electrode based on macrocyclic ligand. Electroanalysis 2001, 13, 1209-1214.

290. Shamsipur, M.; Kazemi, S. Y. A PVC-based dibenzodiaza-15-crown-4 membrane potentiometric sensor for Ni(II). Electroanalysis 2000, 12, 1472-1475.

291. Jain, A. K.; Gupta, V. K.; Ganeshpure, P. A.; Raisoni, J. R. New liquid-membrane electrodes used for potential determination of copper and nickel. Anal. Chim. Acta 2005, 553, 177-182.

292. Kumar, K. G.; Poduval, R.; Augustine, P.; John, S.; Saraswathyamma, B. A PVC plasticized sensor for $\mathrm{Ni}(\mathrm{II})$ ion based on a simple ethylenediamine derivative. Anal. Sci. 2006, 22, 13331337.

293. Kumar, K. G.; Poduval, R.; John, S.; Augustine, P. A PVC plasticized membrane sensor for nickel ions. Mikrochim. Acta 2006, 156, 283-287.

294. Pleniceany, M.; Isvoranu, M.; Spinu, C. Liquid membrane ion-selective electrodes for potentiometric dosage of coper and nickel. J. Serb. Chem. Soc. 2005, 70, 269-276.

295. Pleniceanu, M.; Isvoranu, M.; Spinu C. Electroanalytical applications of some mixed complex combinations with Schiff base. Asian J. Chem. 2005, 17, 2129-2136. 
296. Pleniceanu, M.; Isvoranu, M.; Spinu, C. New electrochemical sensors used for potentiometric determination of copper and nickel. J. Indian Chem. Soc. 2002, 79, 884-886.

297. Alizadeh, N.; Ershad, S.; Naeimi, H.; Sharghi, H.; Shamsipur, M. Copper(II)-selective membrane electrode based on a recently synthesized naphthol-derivative Schiff's base. Fresen. J. Anal. Chem. 1999, 365, 511-515.

298. Ganjali, M. R.; Poursaberi, T.; Babaei, L. H. A.; Rouhani, S.; Yousefi, M.; Kargar-Razi, M.; Moghimi, A.; Aghabozorg, H.; Shamsipur, M. Highly selective and sensitive copper(II) membrane coated graphite electrode based on a recently synthesized Schiff's base. Anal. Chim. Acta 2001, 440, 81-87.

299. Poursaberi, T.; Hajiagha-Babaei, L.; Yousefi, M.; Rouhani, S.; Shamsipur, M.; Kargar-Razi, M.; Moghimi, A.; Aghabozorg, H.; Ganjali, M. R. The synthesis of a new thiophene-derivative Schiff's base and its use in preparation of copper-ion selective electrodes. Electroanalysis 2001, 13, 1513-1517.

300. Ganjali, M. R.; Emami, M.; Salavati-Niasari, M. Novel copper(II)-selective sensor based on a new hexadentates Schiff's base. Bull. Korean Chem. Soc. 2002, 23, 1394-1398.

301. Sadeghi, S.; Eslahi, M.; Naseri, M. A.; Naeimi, H.; Sharghi, H.; Shameli, A. Copper ion selective membrane electrodes based on some Schiff base derivatives. Electroanalysis 2003, 15, 1327 1333.

302. Ganjali, M. R.; Golmohammadi, M.; Yousefi, M.; Norouzi, P.; Salavati-Niasari, M.; Javanbakht, M. Novel PVC-based copper(II) membrane sensor based on 2-(1'-(4'-(1'-hydroxy-2"naphthyl)methyleneamino)butyliminomethyl)-1-naphthol. Anal. Sci. 2003, 19, 223-227.

303. Singh, L. P.; Bhatnagar, J. M. Copper(II) selective electrochemical sensor based on Schiff Base complexes. Talanta 2004, 64, 313-319.

304. Fakhari, A. R.; Raji, T. A.; Naeimi, H. Potentiometric determination of copper in copper sulfide ores. Sens. Actuators B, 2005, 104, 317-332.

305. Norouzi, P.; Ganjali, M. R.; Faridbod, F.; Salavati-Niasari, M. Determination of copper in black, red pepper and the waste water samples by a highly selective sensitive $\mathrm{Cu}$ (II) microelectrode based on a new hexadentates Schiff's base. Bull. Kor, Chem. Soc. 2006, 27, 1439-1444.

306. Jeong, D. C.; Lee, H. K.; Jeon, S. Highly copper(II)-selective PVC membrane based on a Schiff base complex of N,N'-bis-pyridin-2-ylmethylene-naphthalene-1,8-diamine as an ionophore. Bull. Korean Chem. Soc. 2006, 27, 1593-1596.

307. Gholivand, M. B.; Rahimi-Nasrabadi, M.; Ganjali, M. R.; Salavati-Niasari, M. Highly selective and sensitive copper membrane electrode based on a new synthesized Schiff base. Talanta 2007, 73, 553-560.

308. Chandra, S.; Singh, C. K.; Agarwal, H.; Agarwal, R. K. A copper(II)-selective PVC membrane electrode based on a macrocyclic ligand, 1,2,5,6,8,11-hexaazacyclododeca-7,12-dione-2,4,8,10tetraene. Anal. Sci. 2007, 23, 683-687.

309. Chandra, S.; Singh, C. K.; Agarwal, H. Polymeric membrane ion-selective electrode for copper(II) ions based on $\mathrm{Cu}-\mathrm{II}-$ cyclohexaneone thiosemicarbazone complex. J. Indian Chem. Soc. 2007, 84, 291-292. 
310. Shamsipur, M.; Mizani, F.; Saboury, A. A.; Sharghi, H.; Khalifeh, R. Highly selective and sensitive membrane sensors for copper(II) ion based on a new benzo-substituted macrocyclic diamide $\quad 6,7,8,9,10$-hexahydro-2H-1,13,4,7,10-benzodioxatriazacyclopenta-decine-3,11(4H, 12H)-dione. Electroanalysis 2007, 19, 587-586.

311. de Oliveira, I. A. M.; Pla-Roca, M.; Escriche, L.; Casabo, J.; Zine, N.; Bausells, J.; Teixidor, F.; Crespo, E.; Errachid, A.; Samitier, J. Novel all-solid-state copper(II) microelectrode based on a dithiomacrocycle as a neutral carrier. Electrochim. Acta 2006, 51, 5070-5074.

312. Ganjali, M. R.; Golmohammadi, M.; Yousefi, M.; Norouzi, P.; Salavati-Niasari, M.; Javanbakht, M. Novel PVC-based copper(II) membrane sensor based on 2-(1'-(4'-(1'-hydroxy-2"naphthyl)methyleneamino)butyl iminomethyl)-1-naphthol. Anal. Sci. 2003, 19, 223-227.

313. Shamsipur, M. Javanbakht, M.; Mousavi, M. F.; Ganjali, M. R.; Lippolis, V.; Garau, A.; Tei, L. Copper(II)-selective membrane electrodes based on some recently synthesized mixed azathioether crowns containing a 1,10-phenanthroline sub-unit. Talanta 2001, 55, 1047-1054.

314. Sadeghi, S.; Vardini, M.T.; Naeimi, H. Copper (II) ion selective liquid membrane electrode based on new Schiff base carrier. Anal. Chim. 2006, 96, 65-74.

315. Zamani, H. A.; Rajabzadeh, G.; Ganjali, M. R.; Khatami, S. M. Highly selective and sensitive copper(II) membrane sensors based on 6-methyl-4-(1-phenylmethylidene) amino-3-thioxo-1,2,4triazin-5-one as a new neutral ionophore. Electroanalysis 2005, 17, 2260-2265.

316. Gupta, V. K.; Goyal, R. N.; Bachheti, N.; Singh, L. P.; Agarwal, S. A copper-selective electrode based on bis(acetylacetone)propylenediimine. Talanta 2005, 68, 193-197.

317. Zamani, H. A.; Rajabzadeh, G.; Firouz, A.; Ariaii-Rad, A. A. Synthesis of 4-amino-6-methyl1,2,4-triazin-5-one-3-thione and its application in construction of a highly copper(II) ionselective electrochemical sensor. JBCS 2005, 16, 1061-1067.

318. Akhond, M.; Ghaedi, M.; Tashkhourian, J. Development of a new copper(II) ion-selective poly(vinyl chloride) membrane electrode based on 2-mercaptobenzoxazole. Bull. Korean Chem. Soc. 2005, 26, 882-886.

319. Shamsipur, M.; Avanes, A.; Javanbakht, M.; Ganjali, M. R.; Sharghi, H. A 9,10-anthraquinone derivative having two propenyl arms as a neutral ionophore for highly selective and sensitive membrane sensors for copper(II) ion. Anal. Sci. 2002, 18, 875-879.

320. Shamsipur, M. Rouhani, S.; Ganjali, M. R.; Eshghi, H.; Sharghi, H. Copper(II)-selective membrane electrode based on a recently synthesized macrocyclic diamide. Microchem. J. 1999, 63, 202-210.

321. Gupta, K. C.; D'Arc, M. J. Performance evaluation of copper ion selective electrode based on cyanocopolymers. Sens. Actuators B 2000, 62, 171-176.

322. Alizadeh, N.; Ershad, S.; Naeimi, H.; Sharghi, H.; Shamsipur, M. Copper(II)-selective membrane electrode based on a recently synthesized naphthol-derivative Schiff's base. Fresen. J. Anal. Chem. 1999, 365, 511-515.

323. Shamsipur, M.; Rouhani, S.; Ganjali, M. R.; Eshghi, H.; Sharghi, H. Copper(II)-selective membrane electrode based on a recently synthesized macrocyclic diamide. Microchem. J. 1999, 63, 202-210. 
324. Gupta, V. K.; Prasad, R.; Kumar, A. Preparation of ethambutol-copper(II) complex and fabrication of PVC based membrane potentiometric sensor for copper. Talanta 2003, 60, 149160.

325. Ganjali, M. R.; Emami, M.; Salavati-Niasari, M. Novel copper(II)-selective sensor based on a new hexadentates Schiff's base. Bull. Korean Chem. Soc. 2002, 23, 1394-1398.

326. Firooz, A. R.; Mazloum, M.; Safari, J.; Amini, M. K. Coated-wire copper(II)-selective electrode based on phenylglyoxal based on phenylglyoxal-alpha-monoxime ionophore. Anal. Bioanal. Chem. 2002, 372, 718-722.

327. Shamsipur, M.; Javanbakht, M.; Mousavi, M. F.; Ganjali, M. R.; Lippolis, V.; Garau, A.; Tei, L. Copper(II)-selective membrane electrodes based on some recently synthesized mixed azathioether crowns containing a 1,10-phenanthroline sub-unit. Talanta 2001, 55, 1047-1054.

328. Ganjali, M. R.; Poursaberi, T.; Babaei, L. H. A.; Rouhani, S.; Yousefi, M.; Kargar-Razi, M.; Moghimi, A.; Aghabozorg, H.; Shamsipur, M. Highly selective and sensitive copper(II) membrane coated graphite electrode based on a recently synthesized Schiff's base. Anal. Chim. Acta 2001, 440, 81-87.

329. Gholivand, M. B.; Nozari, N. Copper(II)-selective electrode using 2,2'-dithiodianiline as neutral carrier. Talanta 2001, 54, 597-602.

330. Saleh, M. B. Copper(II) sensor based on a cephaloridine antibiotic PVC matrix. Anal. Lett. 2000, $33,1501-1512$.

331. Zamani, H. A.; Rajabzadeh, G.; Firouz, A.; Ganjali, M. R. Determination of Copper(II) in Wastewater Electroplating Samples Based on PVC-Membrane Copper(II)-Selective Electrode. $J$. Anal. Chem. 2007, 62, 1080-1087.

332. Javanbakht, M.; Badiei, A.; Ganjali, M. R.; Norouzi, P.; Hasheminasab, A.; Abdouss, M. Use of organofunctionalized nanoporous silica gel to improve the lifetime of carbon paste electrode for determination of copper(II) ions. Anal. Chim. Acta 2007, 601, 172-182.

333. Mahajan, R. K.; Kumar, M.; Sharma, V.; Kaur, I. Silver(I) ion-selective membrane based on Schiff base-p-tert-butylcalix[4]arene. Analyst 2001, 126, 505-507.

334. Ueda, M.; Sakaki, N.; Moriuchi-Kawakami, T.; Shibutani, Y. Potentiometric performance of a dioxime-type Schiff base as a silver-ion recognizable material. Bunseki Kagaku 2001, 50, 619625.

335. Mahajan, R. K.; Kaur, I.; Kumar, M. Silver ion-selective electrodes employing Schiff base p-tertbutyl calix[4]arene derivatives as neutral carriers. Sens. Actuators B 2003, 91, 26-31.

336. Mahajan, R. K.; Kaur, I.; Sharma, V.; Kumar, M. Sensor for silver(I) ion based on Schiff-base-ptertbutylcalix[4] arene. Sensors 2002, 2, 417-423.

337. Mashhadizadeh, M. H.; Mostafavi, A.; Allah-Abadi, H.; Sheikhshoai, I. New Schiff base modified carbon paste and coated wire PVC membrane electrode for silver ion. Sens. Actuators $B$ 2006, 113, 930-936.

338. Zhang, L. N.; Chai, Y. Q.; Yuan, R.; Li, Y.; Ye, G. R. Study of a new selective silver electrode based on 2,3-butanedione-thiosemicarbazid as a neutral carrier. Acta Chim. Sin. 2007, 65, 537541. 
339. Yan, Z. N.; Lu, Y. Q.; Li, X. Silver ion-selective electrodes based on bis(dialkyldithiocarbamates) as neutral ionophores. Sens. Actuators B 2007, 122, 174-181.

340. Mittal, S. K.; Kumar, A. S. K.; Kaur, S.; Kumar, S. Potentiometric performance of 2aminothiophenol based dipodal ionophore as a silver sensing material. Sens. Actuators B 2007, 121, 386-395.

341. Demirel, A.; Dogan, A.; Akkus, G.; Yilmaz, M.; Kilic, E. Silver(I)-selective PVC membrane potentiometric sensor based on a recently synthesized calix[4]arene. Electroanalysis 2006, 18, 1019-1027.

342. Oconnor, K. M.; Henderson, W.; ONeill, E.; Arrigan, D. W. M.; Harris, S. J.; McKervey, M. A.; Svehla, G. Calix[4]arenes in the partial cone conformation as ionophores in silver ion-selective electrodes. Electroanalysis 1997, 9, 311-315.

343. Singh, A. K.; Saxena, P. Silver(I)-selective electrode based on [Bz(2)Oxo(4)(18)dieneS(4)] tetrathia macrocyclic carrier. Anal. Bioanal. Chem. 2006, 385, 90-95.

344. Mahajan, R. K.; Kaur, I.; Kaur, R.; Bhalla, V.; Kumar, M. Calix[4]arene derivatives: Efficient ionophores for silver(I) ion sensors. Bull. Chem. Soc. Japan. 2005, 78, 1635-1640.

345. Ibrahim, H. Carbon paste electrode modified with silver thimerosal for the potentiometric flow injection analysis of silver(I). Anal. Chim. Acta 2005, 545, 158-165.

346. Goldcamp, M. J.; Ashley, K.; Edison, S. E.; Pretty, J.; Shumaker, J. A bis-oxime derivative of diaza-18-crown-6 as an ionophore for silver ion. Electroanalysis 2005, 17, 1015-1018.

347. Mashhadizadeh, M. H.; Shamsipur, M. Silver(I)-selective membrane electrode based on hexathia-18-crown-6. Anal. Chim. Acta 1999, 381, 111-116.

348. Katsu, T.; Xu, D. F. Organoselenide as a novel ionophore for a silver-selective membrane electrode. Anal. Lett. 1998, 31, 1979-1989.

349. Badr, I. H. A. A new neutral carrier for silver ions based on a bis(thiothiazole) derivative and its evaluation in membrane electrodes. Microchim. Acta 2005, 149, 87-94.

350. Ardakani, M. M.; Zare, H. R.; Dehghani, H.; Jalayer, M. Silver(I) ion selective membrane electrode based on derivative of porphine. Bull. Electrochem. 2004, 20, 385-390.

351. Ueda, M.; Saito, T.; Fujimori, K.; Moriuchi, T.; Shibutani, Y. Potentiometric performance of silver ion-selective electrodes based on tridentate Schiff base derivatives. Anal. Sci. 2004, 20 , 1649-1653.

352. Lu, J. Q.; Pang, D. W.; Zeng, X. S.; He, X. W. A new solid-state silver ion-selective electrode based on a novel tweezer-type calixarene derivative. J. Electroanal. Chem. 2004, 568, 37-43.

353. Zare, H. R.; Salavati-Niasari, M.; Memarzadeh, F.; Mazloum, M.; Nasirizadeh, N. Coated wire silver-ion selective electrode based on a N,N'-bis(2-thienylmethylene)-1,2-diaminobenzene. Anal. Sci. 2004, 20, 815-819.

354. Amini, M. K.; Ghaedi, M.; Rafi, A.; Mohamadpoor-Baltork, L.; Niknam, K. Silver selective electrodes based on methyl-2-pyridyl ketone oxime, phenyl-2-pyridyl ketone oxime and bis[2-(ocarboxythiophenoxy)methyl]-4-bromo-1-methoxybenzene carriers. Sens. Actuators B 2003, 96, 669.

355. Ardakani, M. M.; Ensafi, A. A.; Salavati-Niasari. M.; Mirhoseini, H. Silver(I)-selective coatedwire electrode based on an octahydroxycalix[4]arene derivative. Anal. Sci. 2003, 19, 1187-1190. 
356. Gupta, V. K.; Antonijevic, M. M.; Chandra, S.; Agarwal, S. Polystyrene based silver selective electrodes. Sensors 2002, 2, 233-243.

357. Shamsipur, M.; Javanbakht, M.; Ganjali, M. R.; Mousavi, M. F.; Lippolis, V.; Garau, A. Mixed aza-thioether crowns containing a 1,10-phenanthroline sub-unit as neutral ionophores for silver ion. Electroanalysis 2002, 14, 1691-1698.

358. Amini, M. K.; Rafi, A.; Mohammadpoor-Baltork, I. Silver-selective membrane electrodes using 2-mercaptobenzimidazole and 2-mercaptobenzothiazole carriers. Anal. Lett. 2002, 35, 17951809.

359. Mazloum, M.; Salavati-Niasari, M.; Chahooki, S. H. M.; Amini, M. K. Silver-selective coatedwire electrode based on resorc[4]arene neutral carrier. Electroanalysis 2002, 14, 376-381.

360. Lim, S. M.; Chung, H. J.; Paeng, K. J.; Lee, C. H.; Choi, H. N.; Lee, W. Y. Calix[2]furano[2]pyrrole and related compounds as the neutral carrier in silver ion-selective electrode. Anal. Chim. Acta 2002, 453, 81-88.

361. Chen, L. X.; He, X. W.; Zhang, H. Y.; Liu, Y.; Hu, X. B.; Sheng, Y. Selective electrode for silver based on polymer membranes containing exocyclic chalcogen atoms calix[4]arene and crown ether. Anal. Lett. 2001, 34, 2237-2248.

362. Xu. D. F.; Katsu, T. O,O,O-trialkyl phosphorothioates as simple and effective ionophores for silver ion-selective membrane electrodes. Anal. Chim. Acta 2001, 443, 235-240.

363. Sil, A.; Ijeri, V. S.; Srivastava, A. K. Coated-wire silver ion-selective electrode based on silver complex of cyclam. Anal. Sci. 2001, 17, 477-479.

364. Chen, L. X.; Zeng, X. S.; Ju, H. F.; He, X. W.; Zhang, Z. Z. Calixarene derivatives as the sensory molecules for silver ion-selective electrode. Microchem. J. 2000, 65, 129-135.

365. Mahajan, R. K.; Parkash, O. Silver(I) ion selective PVC membrane based on bis-pyridine tetramide macrocycle. Talanta 2000, 52, 691-693.

366. Chen, L. X.; He, X. W.; Zhao, B. T.; Liu, Y. Calixarene derivative as the neutral carrier in silver ion-selective electrode and liquid membrane transport. Anal. Chim. Acta 2000, 417, 51-56.

367. Liu, D.; Liu, J. H.; Tian, D. Y.; Hong, W. L.; Zhou, X. M.; Yu, J. C. Polymeric membrane silverion selective electrodes based on bis(dialkyldithiophosphates). Anal. Chim. Acta 2000, 416, 139144.

368. Shamsipur, M.; Javanbakht, M.; Lippolis, V.; Garau, A.; De Filippo, G.; Ganjali, M. R.; Yari, A. Novel $\mathrm{Ag}^{+}$ion-selective electrodes based on two new mixed azathioether crowns containing a 1, 10-phenanthroline sub-unit. Anal. Chim. Acta 2002, 462, 225-234.

369. Shamsipur, M.; Rouhani, S.; Mohajeri, A.; Ganjali, M. R.; Poursaberi, T. Silver selective PVCmembrane sensors with and without graphite based on C-methylcalix[4]resorcareneoctamethyl ester. Chem. Analitycz. 2003, 48, 947-958.

370. Ganjali, M. R.; Babaeil, L. H.; Taghvaei-Ganjali, S.; Modjallal, A.; Shamsipur, M.; Hosseini, M.; Javanbakht, M. A new cone shaped asymmetrically substituted calix[4]arene as an excellent ionophore in construction of $\mathrm{Ag}(\mathrm{I})$ ion-selective membrane electrode. Bull. Korean Chem. Soc. 2004, 25, 177-181. 
371. Ganjali, M. R. ; Norouzi, P. ; Alizadeh, T. ; Adib, M. Ion Recognition: Synthesis of 2-methyl2,4-di(2-thienyl)-2,3-dihydro-1H-1,5-benzodiazepine and its Application in Construction of a Highly Selective and Sensitive Ag+ Membrane Sensors. J. Braz. Chem. Soc. 2006, 17, 1217.

372. Javanbakht, M.; Ganjali, M. R.; Norouzi, P.; Hashemi-Nasa, A.; Badei, A. R. Carbon Paste Electrode Modified with Functionalized Nanoporous Silica Gel as a New Sensor for Determination of Silver Ion. Eelectroanalysis 2007, 19, 1307-1314.

373. Gupta, V. K.; Agarwal, S.; Jakob, A.; Lang, H. A zinc-selective electrode based on N,N'bis(acetylacetone)ethylenediimine. Sens. Actuators B 2006, 114, 812-818.

374. Zamani, H. A.; Ganjali, M. R.; Pooyamanesh, M. J. Zinc(II) PVC-based membrane sensor based on 5,6-benzo-4,7,13,16,21,24-hexaoxa-1,10-diazabicyclo[8,8,8]hexacos-5-ene. J. Braz. Chem. Soc. 2006, 17, 149-155.

375. Pouretedal, H. R.; Shamsipur, M. A PVC-based cryptand C2(B)22 membrane potentiometric sensor for zinc(II). Fresen. J. Anal. Chem. 1998, 362, 415-418.

376. Shamsipur, M.; Rouhani, S.; Ganjali, M. R.; Sharghi, H.; Eshghi, H. Zinc-selective membrane potentiometric sensor based on a recently synthesized benzo-substituted macrocyclic diamide. Sens. Actuators B, 1999, 59, 30-34.

377. Gupta, V. K.; Al Khayat, M.; Minocha, A.; Kumar, P. Zinc(II)-selective sensors based on dibenzo-24-crown-8 in PVC matrix. Anal. Chim. Acta 2005, 532, 153-158.

378. Gholivand, M. B.; Mozaffari, Y. PVC-based bis(2-nitrophenyl)disulfide sensor for zinc ions. Talanta 2003, 59, 399-407.

379. Jain, A. K.; Sondhi, S. M.; Rajvanshi, S. A PVC based hematoporphyrin IX membrane potentiometric sensor for zinc(II). Electroanalysis 2002, 14, 293-296.

380. Saleh, M. B.; Gaber, A. A. A. Novel zinc ion-selective membrane electrode based on sulipride drug. Electroanalysis 2001, 13, 104-108.

381. Fakhari, A. R.; Alaghemand, M.; Shamsipur, M. Zinc-selective membrane electrode based on 5,6,14,15-dibenzo-1,4-dioxa-8,12-diazacyclopentadecane-5,14-diene. Anal. Lett. 2000, 33, 21692181.

382. Ganjali, M. R.; Zamani, H. A.; Norouzi, P.; Adib, M.; Rezapour, M.; Aceedy, M. Zn ${ }^{2+}$ PVCbased membrane sensor based on 3-[(2-furylmethylene)amino]-2-thioxo-1,3-thiazolidin-4-one. Bull. Korean Chem. Soc. 2005, 26, 579-584.

383. Singh, A. K.; Mehtab, S.; Singh, U. R.; Aggarwal, V. Comparative studies of tridentate sulfur and nitrogen-containing ligands as Ionophores for construction of cadmium ion-selective membrane sensors. Electroanalysis 2007, 19, 1213-1221.

384. Gupta, V. K.; Jain, A. K.; Kumar, P. PVC-based membranes of dicyclohexano-24-crown-8 as Cd(II) selective sensor. Electrochim. Acta 2006, 52, 736-741.

385. Javanbakht, M.; Shabani-Kia, A.; Darvich, M. R.; Ganjali, M. R.; Shamsipur, M. Cadmium(II)selective membrane electrode based on a synthesized tetrol compound. Anal. Chim. Acta 2000, 408, 75-81.

386. Perez-Marin, L.; Ortiz-Macedo, G.; Avila-Perez, P.; Otazo-Sanchez, E.; Lopez-Valdivia, H.; Alonso-Chammaro, J.; Estevez-Hernandez, O. Liquid membrane electrode sensitive to cadmium (II) ions, using 1-furoyl-3-bencyl-3-phenylthiourea as ionophore. Afinidad 1999, 56, 397-401. 
387. Gupta, V. K.; Kumar, P. Cadmium(II)-selective sensors based on dibenzo-24-crown-8 in PVC matrix. Anal. Chim. Acta 1999, 389, 205-212.

388. Mashhadizadeh, M. H.; Sheikhshoaie, I.; Saeid-Nia, S. Asymmetrical Schiff bases as carriers in PVC membrane electrodes for cadmium (II) ions. Electroanalysis 2005, 17, 648-654.

389. Abbas, M. N.; Zahran, E. Novel solid-state cadmium ion-selective electrodes based on its tetraiodo- and tetrabromo-ion pairs with cetylpyridinium. J. Electroanal. Chem. 2005, 576, 205213.

390. Singh, A. K.; Saxena, P.; Singh, R. New cadmium(II)-selective electrode based on a tetraazacyclohexadeca macrocyclic ionophore. Anal. Sci. 2005, 21, 179-181.

391. Gupta, V. K.; Chandra, S.; Mangla, R. Dicyclohexano-18-crown-6 as active material in PVC matrix membrane for the fabrication of cadmium selective potentiometric sensor. Electrochim. Acta 2002, 47, 1579-1586.

392. Shamsipur, M.; Mashhadizadeh, M. H. Cadmium ion-selective electrode based on tetrathia-12crown-4. Talanta 2001, 53, 1065-1071.

393. Gupta, K. C.; D'Arc, M. J. Cadmium ion-selective electrode based on cyanocopolymer. Electroanalysis 2000, 12, 1408-1413.

394. Gupta, K. C.; D'Arc, M. J. Effect of concentration of ion exchanger, plasticizer and molecular weight of cyanocopolymers on selectivity and sensitivity of $\mathrm{Cd}(\mathrm{II})$ ion selective electrode. Talanta 2000, 52, 1087-1103.

395. Zamani, H. A.; Ganjali, M. R.; Adib, M. Cd(II) PVC-Based Membrane Sensor Based on N'-[1(2-furyl)methylidene]-2-furohydrazide. Sens. Lett. 2006, 4, 345-350.

396. Gismera, M. J.; Hueso, D.; Procopio, J. R.; Sevilla, A. T. Ion-selective carbon paste electrode based on tetraethyl thiuram disulfide for copper(II) and mercury(II). Anal. Chim. Acta 2004, 524, 347-353.

397. Mashhadizadeh, M. H.; Sheikhshoaie, I. Mercury(II) ion-selective polymeric membrane sensor based on a recently synthesized Schiff base. Talanta 2003, 60, 73-80.

398. Mahajan, R. K.; Kaur, R.; Kaur, I.; Sharma, V.; Kumar, M. Mercury(II) ion-selective electrodes based on p-tert-butyl calix[4]crowns with imine units. Anal. Sci. 2004, 20, 811-814.

399. Xu, L.; Yuan, R.; Chai.; Y. Q. Mercury(II) ion potentiometric sensor based on a sulfur Schiff's base 1-(2-hydroxy-1,2-diphenylethylidene)thiosemicarbazide as ionophore. Chem. Lett. 2005, 34, 440-441.

400. Saleh, M. B.; Soliman, E. M.; Gaber, A. A. A.; Ahmed, S. A. A novel Hg(II) PVC membrane sensor based on simple ionophore ethylenediamine bis-thiophenecarboxaldehyde. Anal. Lett. 2006, 39, 659-673.

401. Mashhadizadeh, M. H.; Talakesh, M.; Peste, M.; Momeni, A.; Hamidian, H.; Majum, M. A novel modified carbon paste electrode for potentiometric determination of mercury(II) ion. Electroanalysis 2006, 18, 2174-2179.

402. Ganjali, M. R.; Norouzi, P.; Alizadeh, T.; Salavati-Niasari, M. Synthesis of a new hexadendates Schiff's base and its application in the fabrication of a highly selective mercury(II) sensor. Bull. Korean Chem. Soc. 2007, 28, 68-72. 
403. Gismera, M. J.; Procopio, J. R.; Sevilla, M. T. Characterization of mercury-Humic acids interaction by potentiometric titration with a modified carbon paste mercury sensor. Electroanalysis 2007, 19, 1055-1061.

404. Yu, X.; Zhou, Z. D.; Wang, Y.; Liu, Y.; Xie, Q.; Xiao, D. Mercury (II)-selective polymeric membrane electrode based on the 3-[4-(dimethylamino)phenyl]-5-mercapto-1,5diphenylpentanone. Sens Actuators B 2007, 123, 352-358.

405. Gupta, V. K.; Singh, A. K.; Al Khayat, A.; Gupta, B. Neutral carriers based polymeric membrane electrodes for selective determination of mercury (II). Anal. Chim. Acta 2007, 590, 81-90.

406. Khan. A. A.; Inamuddin. Applications of $\mathrm{Hg}(\mathrm{II})$ sensitive polyaniline $\mathrm{Sn}(\mathrm{IV})$ phosphate composite cation-exchange material in determination of $\mathrm{Hg}^{2+}$ from aqueous solutions and in making ion-selective membrane electrode. Sens. Actuators B 2006, 120, 10-18.

407. Hassan, S. S. M.; Mahmoud, W. H.; Mohamed, A. H. K.; Kelany, A. E. Mercury(II) ion-selective polymeric membrane sensors for analysis of mercury in hazardous wastes. Anal. Sci. 2006, 22, 877-881.

408. Ye, G. R.; Chai, Y. Q.; Yuan, R.; Dai, J. Y. A mercury(II) ion-selective electrode based on N,Ndimethylformamide-salicylacylhydrazone as a neutral carrier. Anal. Sci. 2006, 22, 579-582.

409. Othman, A. M. Potentiometric determination of mercury(II) using a tribromomercuraterhodamine BPVC membrane sensor. Int. J. Environ.Anal Chem. 2006, 86, 367-379.

410. Gupta, V. K.; Chandra, S.; Lang, H. A highly selective mercury electrode based on a diamine donor ligand. Talanta 2005, 66, 575-580.

411. Gupta, V. K.; Jain, S.; Khurana, U. A PVC-based pentathia-15-crown-5 membrane potentiometric sensor for mercury(II). Electroanalysis 1997, 9, 478-480.

412. Jain. A. K.; Sondhi, S. M.; Sharma, V. K. Synthesis, characterization and $\mathrm{Hg}(\mathrm{II})$ Ion selectivity of 1-(2-nitro-4-methylphenyl)-6-methyl-6-methoxy-1,4,5,6-tetrahydro-pyrimidine-2-(3H)thione (TPT). Electroanalysis 2000, 12, 301-305.

413. Javanbakht, M.; Ganjali, M. R.; Eshghi, H.; Sharghi, H.; Shamsipur, M. Mercury(II) ionselective electrode based on dibenzodiazathia-18-crown-6-dione. Electroanalysis 1999, 11, 8184.

414. Lu, J. Q.; Zeng, X. S.; Ai, J.; Pang, D. W. A solid-state ion-selective electrode for mercury with tweezer-type calix[4]arene derivative as the probe on PVC film. Chem. J. Chin Univ-Chin. 2005, 26, 238-240.

415. Singh, A. K.; Bhattacharjee, G.; Singh, R. Mercury(II)-selective membrane electrode using tetrathia-diazacyclotetradeca-2,9-diene as neutral carrier. Sens. Actuators B, 2004, 99, 36-41.

416. Lu, J. Q.; Tong, X. Q.; He, X. W. A mercury ion-selective electrode based on a calixarene derivative containing the thiazole azo group. J. Electroanal. Chem. 2003, 540, 111-117.

417. Mahajan, R. K.; Kaur, I.; Lobana, T. S. A mercury(II) ion-selective electrode based on neutral salicylaldehyde thiosemicarbazone. Talanta 2003, 59, 101-105.

418. Hassan, S. S. M.; Saleh, M. B.; Gaber, A. A. A.; Mehheimer, R. A. H.; Kream, N. A. A. Novel mercury (II) ion-selective polymeric membrane sensor based on ethyl-2-benzoyl-2phenylcarbamoyl acetate. Talanta 2000, 53, 285-293. 
419. Perez-Marin, L.; Otazo-Sanchez, E.; Macedo-Miranda, G.; Avila-Perez, P.; Chamaro, J. A.; Lopez-Valdivia, H. Mercury(II) ion-selective electrode. Study of 1,3-diphenylthiourea as ionophore. Analyst 2000, 125, 1787-1790.

420. Fakhari, A. R.; PVC-based hexathia-18-crown-6-tetraone sensor for mercury(II) ions. Anal. Chem. 1997, 69, 3693-3696.

421. Faridbod, F.; Ganjali, M. R.; Norouzi, P. Schiff's Bases and Crown Ethers as Supramolecular Sensing Materials in Construction of the Potentiometric membrane. Sensors 2008, 8, 1645-1703.

422. Shamsipur, M.; Yousefi, M.; Hosseini, M.; Ganjali, M. R. Lanthanum(III) PVC membrane electrodes based on 1,3,5-trithiacyclohexane. Anal. Chem. 2002, 74, 5538-5543.

423. Gupta, V. K.; Jain, S.; Chandra, S. Chemical sensor for lanthanum(III) determination using azacrown as ionophore in poly(vinyl chloride) matrix. Anal. Chim. Acta 2003, 486, 199-207.

424. Khalil, S. Ion-selective electrode for lanthanum determination in standard samples. Anal. Lett. 2003, 36 1335-1349.

425. Ganjali, M. R.; Daftari, A.; Rezapour, M.; Puorsaberi, T.; Hahgoo, S. Gliclazide as novel carrier in construction of PVC-based La(III)-selective membrane sensor. Talanta 2003, 59, 613-619.

426. Ganjali, M. R.; Norouzi, P.; Shamsolahrari, L.; Ahmadi, A. PPb level monitoring of lanthanium by a novel PCV-membrane sensor based on 4-methyl-2-hydrazinobenzothiazole. Sens. Actuators B 2006, 114, 713-719.

427. Ganjali, M. R.; Qomi, M.; Daftari, A.; Norouzi, P.; Salavati-Niasari, M.; Rabbani, M. Novel lanthanum(III) membrane sensor based on a new N-S Schiff's base. Sens. Actuators B 2004, 98, 92-96.

428. Ganjali, M. R.; Norouzi, P.; Alizadeh, T.; Adib, M. Application of 2-\{[(8-amino-1-naphtyl) imino] methyl $\}$ phenol as a neutral ionophore in the construction of a $\mathrm{La}$ (III) ion-selective sensor. Anal. Chim. Acta 2006, 576, 275-282.

429. Shamsipur, M.; Ershad, S.; Samadi, N.; Esmaeilbeig, A. R.; Kia, R.; Abdolmaleki, A. Polymeric membrane lanthanum(III)-selective electrode based on N,N'-adipylbis(5-plenylazosalicylaldehyde hydrazone). Electroanalysis 2005, 17, 1828-1834.

430. Ganjali, M. R.; Matloobi, P.; Ghorbani, M.; Norouzi, P.; Salavati-Niasari, M. La(III) selective membrane sensor based on a new N-N Schiff's base. Bull. Korean Chem. Soc. 2005, 26, 38-42.

431. Akhond, M.; Najafi, M.B.; Tashkhourian, J. Lanthanum-selective membrane electrode based on 2,2'-dithiodipyridine. Anal. Chim. Acta, 2005, 531, 179-184.

432. Ganjali, M. R.; Akbar, V.; Ghorbani, M.; Norouzi, P.; Ahmadi, A. Fluoride determination in some mouth wash preparations by a novel La(III) graphite coated membrane sensor based on amitraz. Anal. Chim. Acta 2005, 531, 185-191.

433. Ganjali, M. R.; Gholivand, M. B.; Rahimi-Nasrabadi, M.; Maddah, B.; Salavati-Niasari, M.; Ahmadi, F. Synthesis of a new octadentates Schiff's base and its application in construction of a highly selective and sensitive La(III) membrane sensor. Sensor Lett. 2006, 4, 356-363.

434. Ganjali, M. R.; Norouzi, P.; Yousefian, N.; Faridbod, F.; Adib, M. sub-micro molar monitoring of $\mathrm{La}^{3+}$ by a novel lanthanum PVC-based membrane sensor based on 3-hydroxy-N'-(pyridin-2ylmethylene)-2-naphthohydrazide. Bull. Korean Chem. Soc. 2006, 27, 1581-1586. 
435. Zamani, H. A.; Ganjali, M. R.; Norouzi, P.; Adib, M.; Aceedy, M. Synthesis of N'-(1-pyridin-2ylmethylene)-2-furohydrazide and its application in construction of a highly selective PVC-based membrane sensor for La(III) ions. Anal. Sci. 2006, 22, 943-948.

436. Ganjali, M. R.; Norouzi, P.; Alizadeh, T.; Tadjarodi, A.; Hanifehpour, Y. Construction of a highly selective and sensitive La(III) sensor based on N-(2-pyridyl)-N'-(4-methoxyphenyl)-thiourea for nano level monitoring of $\mathrm{La}(\mathrm{III})$ ions. Electroanalysis 2006, 18, 1091-1096.

437. Shamsipur, M.; Yousefi, M.; Ganjali, M. R. PVC-based 1;3;5-trithiane sensor for cerium(III) ions. Anal. Chem. 2000, 72, 2391-2394.

438. Shamsipur, M.; Yousefi, M.; Hosseini, M.; Ganjali, M. R. PVC-based 1;3;5-trithiane coated graphite electrode for determination of cerium(III) ions. Anal. Lett. 2001, 34, 2249-2261.

439. Gaber, A. A. A. A novel PVC membrane sensor for selective determination of cerium(III) ions. Anal. Lett. 2003, 36, 2585-2596.

440. Karami, H.; Mousavi, M. F.; Shamsipur, M.; Yavari, I.; Alizadeh, A. A. A new ion-selective electrode for potentiometric determination of Ce(III). Anal. Lett. 2003, 36, 1065-1078.

441. Akhond, M.; Najafi, M. B. Tashkhourian; J. A new cerium(III)-selective membrane electrode based on 2-aminobenzothiazole. Sens. Actuators B 2004, 99, 410-414.

442. Gupta, V. K.; Singh, A. K.; Gupta, B. A cerium(III) selective polyvinyl chloride membrane sensor based on a Schiff base complex of N,N'-bis[2-(salicylideneamino)ethyl]ethane-1,2diamine. Anal. Chim. Acta 2006, 575, 198-204.

443. Pouretedal, H. R.; Semnani, A.; Keshavarz, M. H. Cerium(III) ion-selective electrodes based on 1,4,7-trithiacyclononane. Turk. J. Chem. 2006, 30,711-721.

444. Saleh, M. B.; Gaber, A. A. A.; Khalaf, M. M. R.; Tawfeek, A. M. A new ion-selective electrode for potentiometric determination of Ce(III) ions. Sens. Actuators B, 2006, 119, 275-281.

445. Zamani, H. A.; Ganjali, M. R.; Adib, M. Construction of a highly selective PVC-based membrane sensor for Ce(III) ions; Sen. Actuators B, 2007, 120, 545-550.

446. Ganjali, M. R.; Mirnaghi, F.; Norouzi, P.; Adib, M. Novel Pr(III)-selective membrane sensor based on a new hydrazide derivative. Sens. Actuators B, 2006, 115, 374-378.

447. Ganjali, M. R.; Norouzi, P.; Mirnaghi, F. S.; Riahi, S.; Faridbod, F. Lanthanide Recognition: Monitoring of Praseodymium(III) by a Novel Praseodymium(III) Microsensor Based on N(Pyridin-2-Ylmethylene)Benzohydrazide. IEEE Sens. J. 2007, 7, 1138-1144.

448. Norouzi, P.; Ganjali, M. R.; Ahmadalinezhad, A.; Adib, M. Novel neodymium(III) membrane sensor based on N-(2-furylmethylene) pyridine-2,6-diamine. J. Braz. Chem. Soc. 2006, 17, 13091315 .

449. Ganjali, M. R.; Ahmadalinezhad, A.; Norouzi, P.; Adib, M. Nd(III)-PVC membrane sensor based on 2-\{[(6- aminopyridin-2-yl)imino] methyl $\}$ phenol. J. Appl. Electrochem. 2006, 36, 931-936.

450. Shamsipur, M.; Hosseini, M.; Alizadeh, K.; Mousavi, M. F.; Garau, A.; Lippolis, V.; Yari, A. PVC membrane potentiometric sensor based on 5-pyridino-2,8-dithia[9](2,9)-1,10phenanthroline-phane for selective determination of neodymium(III). Anal Chem. 2005, 77, 276283. 
451. Behmadi, H.; Zamani, H. A.; Ganjali, M. R.; Norouzi, P. Determination of neodymium(III) ions in soil and sediment samples by a novel neodymium(III) sensor based on benzyl bisthiosemicarbazone. Electrochim. Acta 2007, 53, 1870-1876.

452. Shamsipur, M. Hosseini, M. Alizadeh, K. Eskandari, M. M. Sharghi, H. Mousavi, M. F. Ganjali, M. R. Polymeric membrane and coated graphite samarium(III)- selective electrodes based on isopropyl 2-[(isopropoxycarbothioyl)disulfanyl]ethanethioate. Anal. Chim. Acta 2003, 486, 9399.

453. Ganjali, M. R. Pourjavid, M. R. Rezapour, M. Haghgoo, S. Novel samarium(III) selective membrane sensor based on glipizid. Sens. Actuators B 2003, 89, 21-26.

454. Ganjali, M. R. Rezapour, M. Pourjavid, M. R. Haghgoo, S. ppt level detection of samarium(III) with a coated graphite sensor based on an antibiotic. Anal. Sci. 2004, 20, 1007-1011.

455. Zamani, H. A.; Ganjali, M. R.; Adib, M. Fabrication of a new samarium(III) ion-selective electrode based on 3-\{[2-oxo-1(2H)-acenaphthylenyliden]amino $\}$-2-thioxo-1,3-thiazolidin-4-one, J. Braz. Chem. Soc. 2007, 18, 215-222

457. Saleh M. B.; Gaber, A. A. A.; Khalaf, M. M. R.; Tawfeek, A. M. Novel Sm(III) ion-selective polymeric membrane sensor based on spiro[oxirane-pyrazolidinedione] derivative ionophore. Anal. Lett. 2006, 39, 17-31.

458. Ganjali, M. R.; Rahimi, M.; Maddah, B.; Moghimi, A.; Borhany, S. An Eu(III) sensor based on N,N-diethyl-N-(4-hydroxy-6-methylpyridin-2-yl)guanidine. Anal. Sci. 2004, 20, 1427-1431.

459. Ganjali, M. R.; Daftari, A.; Faridbod, F.; Norouzi, P.; Salavati-Niasari, M. Fabrication of a highly selective $\mathrm{Eu}(\mathrm{III})$ membrane sensor based on a new S-N hexadentates Schiff's base. Sens. Actuators $B$, 2007, 120, 673-678.

460. Zamani, H. A.; Rajabzadeh, G.; Ganjali, M. R. A new europium(III) PVC membrane potentiometric sensor based on 4-(2-hydroxybenzylideneamino)-6-methyl-3-thioxo-3,4-dihydro1,2,4-triazin-5(2H)-one. Bull. Chem. Soc. Japan 2007, 80, 172-177.

461. Ganjali, M. R.; Daftari, A.; Faridbod, F.; Norouzi, P.; Salavati-Niasari, M. Fabrication of a highly selective Eu(III) membrane sensor based on a new S-N hexadentates Schiff's base. Sens. Actuators B 2007, 120, 673-678.

462. Ganjali, M. R.; Tahami, M.; Shamsipur, M.; Poursaberi, T.; Haghgoo, S.; Hosseini, M. Novel gadolinium PVC-based membrane sensor based on omeprazole as an antibiotic. Electroanalysis 2003, 15, 1038-1042.

463. Ganjali, M. R.; Rezapour, M.; Norouzi, P.; Salavati-Niasari, M. A new pentadentate S-N Schiff's base as a novel ionophore in construction of a novel Gd (III) membrane sensor. Electroanalysis, 2005, 17, 2032-2036.

464. Ganjali, M. R.; Norouzi, P.; Alizadeh, T.; Tajarodi, A.; Hanifehpour, Y. Fabrication of a highly selective and sensitive Gd(III)-PVC membrane sensor based on N-(2-pyridyl)-N'-(4nitrophenyl)thiourea. Sens. Actuators B, 2007, 120, 487-493.

465. Zamani, H. A.; Rajabzadeh, G.; Ganjali, M. R.; Norouzi, P. Synthesis of 6-Methyl-4-\{[1-(2thienyl)methylidene]amino 3-thioxo-3,4-dihydro-1,2,4-triazin-5-(2H)-one and Its Use in Fabrication of a Potentiometric PVC Membrane Electrode for Selective Determination of Gadolinium(III) ions. Anal. Chim. Acta 2007, 598, 51-57. 
466. Ganjali, M. R.; Ghesmi, A.; Hosseini, M.; Pourjavid, M. R.; Rezapour, M.; Shamsipur, M.; Salavati-Niasari, M. Novel terbium(III) sensor based on a new bis-pyrrolidene Schiff's base. Sens. Actuators B, 2005, 105, 334-339.

467. Zamani, H. A.; Rajabzadeh, G.; Ganjali, M. R. Construction of a novel potentiometric terbium(III) membrane sensor and its alication for the determination of terbium ion in binary mixture and fluoride ion in a mouth wash preparation. J. Braz. Chem. Soc. 2006, 17, 1297-1303.

468. Ganjali, M. R.; Ravanshad, J.; Hosseini, M.; Salavati-Niasari, M.; Pourjavid, M. R.; Baezzate, M. R. Novel Dy(III) sensor based on a new bis-pyrrolidene Schiff's base. Electroanalysis 2004, 16, 1771-1776.

469. Ganjali, M. R.; Dodangeh, M.; Ghorbani, H.; Norouzi, P.; Adib, M. ppb level monitoring of Dy(III) ions by a highly sensitive and selective Dy(III) sensor based on a new asymmetrical Schiff's base. Anal. Lett. 2006, 39, 495-506.

470. Zamani, H. A.; Ganjali, M. R.; Seifi, N. Dysprosium(III) ion-selective electrochemical sensor based on 6-hydrazino-1,5-diphenyl-6,7-dihydropyrazolo-[3,4-d] pyrimidine-4(5H)-imine. Collect. Czech. Chem. Commun. 2007, 72, 1189-1206.

471. Ganjali, M. R.; Norouzi, P.; Faridbod, F.; Hajiabdollah, N.; Larijani, B. Hanifehpour, Y. Procaine as a sensing material for determination of Dysprosium(III) ions in presence of other rare-earth elements in biological and environmental samples. Anal. Lett. 2007, 40, 2544-2561.

472. Ganjali, M. R.; Norouzi, P.; Adib, M.; Ahmadalinezhad A. A novel Holmium(III) membrane sensor based on N-(1-thien-2-ylmethylene)-1,3-benzothiazol-2-amine. Anal. Lett. 2006, 39, 1075-1086.

473. Ganjali, M. R.; Rasoolipour, S.; Rezapour, M.; Norouzi, P.; Amirnasrb, M.; Meghdadi, S. A Novel Ho(III) Sensor based on N,N'-Bis(2- pyridinecarboxamide)-1,2-benzene as a Neutral Ion Carrier. J. Braz. Chem. Soc. 2006, 17, 1211-1216.

474. Ganjali, M. R.; Rasoolipour, S.; Rezapour, M.; Norouzi, P.; Amirnasr, M.; Meghdadi, S. Application of $\mathrm{N}, \mathrm{N}^{\prime}$-bis(2-quinolinecarboxamide)-1,2-benzene as an ionophore in the construction of a novel polymeric membrane sensor for the selective monitoring of the holmium(III) concentration. Sens. Actuators B 2006, 119, 89-93.

475. Zamani, H. A.; Ganjali, M. R.; Meghdadi, S. Fabrication of a Novel Holmium(III) PVC Membrane Sensor Based on 4-Chloro-1,2-bis(2-pyridinecarboxamido)benzene as a Neutral Ionophore. J. Appl. Electrochem. 2007, 37, 853-859.

476. Ganjali, M. R.; Faridbod, F.; Norouzi, P.; Adib, M. A novel Er(III) sensor based on a new hydrazone for the monitoring of Er(III) ions. Sens. Actuators B, 2006, 120, 119-124.

477. Ganjali, M. R.; Rezapour, M.; Rasoolipour, S.; Norouzi, P.; Adib, M. Application of Pyridine-2carbaldehyde-2-(4-methyl-1,3-benzo thiazol-2-yl)hydrazone as a Neutral Ionophore in the Construction of a Novel Er(III) Sensor. J. Braz. Chem. Soc. 2007, 18, 352-358.

478. Ganjali, M. R.; Rasoolipour, S.; Rezapour, M.; Norouzi, P.; Adib, M. Synthesis of thiophene-2carbaldehyde-(7-methyl-1,3-benzothiazol-2-yl)hydrazone and its alication as an ionophore in the construction of a novel thulium(III) selective membrane sensor. Electrochem. Commun. 2005, 7 , 989-994. 
479. Ganjali, M. R.; Norouzi, P.; Tamaddon, A.; Husain, S. W. Novel Tm(III) membrane sensor based on 2,2 '-dianiline disulfide and its application for the fluoride monitoring of mouth wash preparations. Bull. Korean Chem. Soc. 2006, 27, 1418-1422.

480. Ganjali, M. R.; Norouzi, P.; Akbari-Adergani, B. Thulium(III) Ions Monitoring by a Novel Thulium(III) Microelectrode Based on a S-N Schiff Base. Electroanalysis 2007, 19, 1145-1151.

481. Ganjali, M. R.; Norouzi, P.; Tamaddon, A.; Adib, M. Nano-level monitoring of ytterbium(III) by a novel ytterbium(III) membrane sensor based on 3-hydroxy-N '-[(2-hydroxyphenyl) methylene]-2naphthohydrazide. Sens. Actuators B 2006, 114, 855-860.

482. Zamani, H. A.; Rajabzadeh, G.; Ganjali, M. R. A new ytterbium(III) PVC membrane electrode based on 6methy-4-\{[1-(1H-pyrrol-2-yl)methylidene]amino\}-3-thioxo-3,4-dihydro-1,2,4-triazin-5(2H)-one. Talanta 2007, 72, 1093-1099.

483. Ganjali, M. R.; Norouzi, P.; Ghorbani, H.; Larijani, B.; Tadjarodi, A.; Hanifehpour, Y. Lanthanide recognition: Fabrication of an Ytterbium PVC membrane sensor based on N-(6-picolyl)-N'-(4metoxyphenyl) thiourea (PMPT). Can. Soc. J. Anal. Sci. Spect. 2006, 51, 279-287.

484. Ganjali, M. R.; Naji, L.; Poursaberi, T.; Shamsipur, M.; Haghgoo, S. Ytterbium(III)-selective membrane electrode based on cefixime. Anal. Chim. Acta 2003, 475, 59-66.

485. Ganjali, M. R.; Rasoolipour, S.; Rezapour, M.; Norouzi, P.; Tajarodi, A.; Hanifehpour, Y. Novel ytterbium(III) selective membrane sensor based on N-(2-pyridyl)-N'-(2-methoxyphenyl)-thiourea as an excellent carrier and its alication to determination of fluoride in mouth wash preparation samples. Electroanalysis 2005, 17, 1534-1539.

486. Ganjali, M. R.; Tamaddon, A.; Norouzi, P.; Adib, M. Novel Lu(III) membrane sensor based on a new asymmetrically S-N Schiff's base. Sens. Actuators B 2006, 120, 194-199.

487. Ganjali, M. R.; Norouzi, P.; Akbari-Adergani, B.; Riahi, S. Larijani, B. An Asymetric Lutetium(III) Microsensor Based on N-(2-Furylmethylene) Pyridine-2,6-Diamine for Determination of Lutetium(III) Ions. Anal. Lett. 2007, 40, 1923-1938.

488. Ganjali, M. R.; Norouzi, P.; Faridbod, F.; Riahi, S. Highly Selective and Sensitive Th ${ }^{4+}-\mathrm{PVC}-$ Based Membrane Sensor based on 2-(diphenylphosphorothioyl)-N', N'-diphenylacetamide. $J$. Appl. Electrochem. 2007, 37, 827-833.

489. Chandra, S.; Agarwal, H.; Singh, C. K.; Sindhu, S. K.; Kumar, P.; A polymeric membrane ion selective electrode based on organic-inorganic composite ion exchanger for the determination of thorium(IV). Ind. J. Chem. 2005, 44, 2060-2063.

490. Mittal, S. K.; Singh, P. P. Thorium selective electrode using zirconium phosphoborate as electroactive material. Ind. J. Chem. A, 1995, 34, 1009-1011.

491. Arida, H. A.; Ahmed, M. A.; El-Saied, A. M. A novel coated graphite rod Th(IV) ion selective electrode based on thorium oxinate complex and its application. Sensors 2003, 3, 424-437.

492. Hassanzadeh, P.; Yaftian, M. R.; Bahari, Z.; Matt, D. A coated graphite thorium-ion selective potentiometric sensor based on a calix[4]arene bearing phosphoryl groups. J. Chin. Chem. Soc. 2006, 53, 1113-1118.

493. Maj-Zurawska, M.; Ziemianek, D.; Mikolajczuk, A.; Mieczkowski, J.; Lewenstam, A.; Hulanicki, A.; Sokalski, T. Anal. Bioanal. Chem. 2003, 376, 524-526. 
494. Zeng, Y. L.; Tang, C. R. Hydrogencarbonate anion electrode based on long chain S-akyl diphenylthiocarbazone. Chin. J. Anal. Chem. 2001, 29, 799-801.

495. Ganjali, M. R.; Rezapour, M.; Pourjavid, M. R.; Salavati-Niasari, M. Highly selective PVCmembrane electrodes based on Co(II)-Salen for determination of nitrite ion. Anal. Sci. 2003, 19, 1127-1131.

496. Ganjali, M. R.; Shirvani-Arani, S.; Norouzi, P.; Rezapour, M.; Salavati-Niasari, M.; Novel nitrite membrane sensor based on cobalt(II) salophen for selective monitoring of nitrite ions in biological samples. Mikrochim. Acta 2004, 146, 35-41.

497. Shamsipur, M.; Javanbakht, M.; Hassaninejad, A. R.; Sharghi, H.; Ganjali, M. R.; Mousavi, M. F. Highly selective PVC-membrane electrodes based on three derivatives of (Tetraphenylporphyrinato) cobalt(III) acetate for determination of trace amounts of nitrite ion. Electroanalysis 2003, 15, 1251-1259.

498. Hu, W. P.; Dong, X. Z.; He, Z. J. The determination of nitrite by catalytic kinetic potentiometric method using methyl violet selective electrode. Chin. J. Anal. Chem. 2004, 32, 765-768.

499. Schazmann, B.; Diamond, D. Improved nitrate sensing using ion selective electrodes based on urea-calixarene ionophores. New J. Chem. 2007, 31, 587-592.

500. Watts, A. S.; Gavalas, V. G.; Cammers, A.; Andrada, P. S.; Alajarin, M.; Bachas, L. G. Nitrateselective electrode based on a cyclic bis-thiourea ionophore. Sens. Actuators B 2007, 121, 200207.

501. Alvarez-Romero, G. A.; Palomar-Pardave, M. E.; Ramirez-Silva, M. T. Development of a novel nitrate-selective composite sensor based on doped polypyrrole. Anal. Bioanal. Chem. 2007, 387, 1533-1541.

502. Hassan, S. S. M.; Sayour, H. E. M.; Al-Mehrezi, S. S. A novel planar miniaturized potentiometric sensor for flow injection analysis of nitrates in wastewaters, fertilizers and pharmaceuticals. Anal. Chim. Acta 2007, 581, 13-18.

503. Khripoun, G. A. Volkova, E. A.; Liseenkov, A. V.; Mikhelson, K. N. Nitrate-selective solid contact electrodes with poly(3-octylthiophene) and poly(aniline) as ion-to-electron transducers buffered with electron-ion-exchanging resin. Electroanalysis 2006, 18, 1322-1328.

504. Marin, L. P.; Perez, M.; Chamarro J. A.; Tacoronte, J. E. Construction and evaluation of an ionselective electrode to nitrate, of liquid membrane, applied on a support of conductive epoxy resin. Afinidad 1998, 55, 179-182.

505. Ortuno, J. A.; Exposito, R.; Sanchez-Pedreno, C.; Albero, M. I.; Espinosa, A. A nitrate-selective electrode based on a tris(2-aminoethyl)amine triamide derivative receptor. Anal. Chim. Acta 2004, 525, 231-237.

506. Santos, E.; Montenegro, M.; Couto, C.; Araujo, A. N.; Pimentel, M. F.; da Silva, V. L. Sequential injection analysis of chloride and nitrate in waters with improved accuracy using potentiometric detection. Talanta 2004, 63, 721-727.

507. Perez, M. D. A.; Nodarse, I. C.; Yazdani-Pedram, M. Construction of a polymeric liquidmembrane ion-selective electrode (ISE) and its application for determination of nitrate in tomatoes. J. Chil. Chem. Soc. 2004, 49, 31-34. 
508. Le Goff, T.; Braven, J. Ebdon, L.; Scholefield, D. Automatic continuous river monitoring of nitrate using a novel ion-selective electrode. J. Environ. Monit. 2003, 5, 353-358.

509. Bendikov, T. A.; Kim, J.; Harmon, T. C. Development and environmental application of a nitrate selective microsensor based on doped polypyrrole films. Sens. Actuators B 2005, 106, 512-517.

510. Perez-Olmos, R.; Rios, A.; Fernandez, J. R.; Lapa, R. A. S.; Lima, J. L. F. C. Construction and evaluation of ion selective electrodes for nitrate with a summing operational amplifier. Application to tobacco analysis. Talanta 2001, 53, 741-748.

511. Kim, H. J.; Hummel, J. W.; Sudduth, K. A.; Birrell, S. J. Evaluation of phosphate ion-selective membranes and cobalt-based electrodes for soil nutrient sensing. T. Asabe 2007, 50, 415-425.

512. Wroblewski, W.; Wojciechowski, K.; Dybko, A.; Brzozka, Z.; Egberink, R. J. M.; Snellink-Ruel, B. H. M.; Reinhoudt, D. N. Uranyl salophenes as ionophores for phosphate-selective electrodes. Sens. Actuators B 2000, 68, 313-318.

513. Kivlehan, F.; Mace, W. J.; Moynihan, H. A.; Arrigan, D. W. M. Potentiometric evaluation of calix[4]arene anion receptors in membrane electrodes: Phosphate detection. Anal. Chim. Acta 2007, 585, 154-160.

514. Ganjali, M. R.; Mizani, F, Emami, M, Salavati-Niasari, M.; Shamsipur, M.; Yousefi, M, Javanbakht, M. Electroanalysis 2003, 15, 139.

515. Jain, A. K.; Gupta, V. K.; Raisoni, J. R. A newly synthesized macrocyclic dithioxamide receptor for phosphate sensing. Talanta 2006, 69, 1007-1012.

516. Ganjali, M. R.; Norouzi, P.; Ghomi, M.; Salavati-Niasari, M. Highly selective and sensitive monohydrogen phosphate membrane sensor based on molybdenum acetylacetonate. Anal. Chim. Acta 2006, 567, 196-201.

517. Ganjali, M.R.; Norouzi P.; Hatambeygi, N.; Salavati-Niasari, M. Anion recognition: Fabrication of a highly selective and sensitive HPO4 2-PVC sensor based on a oxo-molybdenum methylsalen. J. Brazil. Chem. Soc. 2006, 17, 859-865.

518. Ganjali, M.R.; Mizani, F.; Emami, M.; Salavati-Niasari, M.; Shamsipur, M.; Yousefi, M.; Javanbakht, M,. Novel liquid membrane electrode for selective determination of monohydrogenphosphate. Electroanalysis 2003, 15, 139-144.

519. Liu, D.; Chen, W. C.; Yang, R. H.; Shen, G. L.; Yu, R. Q. Polymeric membrane phosphate sensitive electrode based on binuclear organotin compound. Anal. Chim. Acta 1997, 338, 209214.

520. Fibbioli, M.; Berger, M.; Schmidtchen, F. P.; Pretsch, E. Polymeric membrane electrodes for monohydrogen phosphate and sulfate. Anal. Chem. 2000, 72, 156-160.

521. Le Goff, T.; Braven, J.; Ebdon, L.; Scholefield, D. Phosphate-selective electrodes containing immobilised ionophores. Anal. Chim. Acta 2004, 510, 175-182.

522. Ganjali, M. R.; Highly selective and sensitive chromium(III) membrane sensors based on a new tridentate Schiff's base. Anal. Chim. Acta 2006, 569, 35-41.

523. Goto, Y.; Miyazaki, K.; Hattori, H.; Yuchi, A. Phosphate-selective electrode using bis(pentafluorobenzyl) tin(IV) dibromide as carrier. Bunseki Kagaku 2003, 52, 707-711. 
524. De Marco, R.; Phan, C. Determination of phosphate in hydroponic nutrient solutions using flow injection potentiometry and a cobalt-wire phosphate ion-selective electrode. Talanta 2003, 60, 1215-1221.

525. Sasaki, S.; Ozawa, S.; Citterio, D.; Yamada, K.; Suzuki, K. Organic tin compounds combined with anionic additives - an ionophore system leading to a phosphate ion-selective electrode? Talanta 2004, 63, 131-134.

526. Hassan, S. S. M.; Hamza, M. S. A.; Kelany, A. E. A novel spectrophotometric method for batch and flow injection determination of cyanide in electroplating wastewater. Talanta 2007, 71, 1088-1095.

527. Marin, M. A. B.; da Silva, R. C.; Lehmkuhl, A.; da Silva, J. B. B.; Ganzarolli, E. M.; de Queiroz, R. R. U. Automatic system for sequential determination of free and total cyanide using homogeneous membrane tubular ion-selective electrode. Quim. Nova 2000, 23, 23-29.

528. Ganjali, M. R. Highly selective thiocyanate poly(vinyl chloride) membrane electrode based on a cadmium-Schiff's base complex. Fresen. J. Anal. Chem. 2001, 370, 1091-1095.

529. Ardakani, M. M.; Salvati-Niassari, M.; Sadeghi, A. Novel selective thiocyanate PVC membrane electrode based on new Schiff base complex of 2.2-[(1,3-dimethyl-1,3-propanediylidene)dinitrilo]bis-benzenethiolato cadmium(II). New J. Chem. 2004, 28, 595-599.

530. Ardakani, M.M. Sadeghi, A. and Salavati-Niasari, M. Highly selective thiocyanate membrane electrode based on butane-2,3-dione bis(salicylhydrazonato)zinc(II) complex. Talanta 2005, 66, 837-843.

531. Gui, G. F.; Chai, Y. Q.; Yuan, R.; Sun, A. L.; Wang, F. C. Study on new highly selective thiocyanate electrode based on dinuclear copper complex as neutral carriers. Acta Chim. Sin. 2006, 64, 2185-2189.

532. Ardakani, M. M.; Jamshidpour, M.; Naeimi, H.; Moradi, L. Thiocyanate ion-selective PVC membrane electrode based on N,N '-ethylene-bis(4-methylsalicylidineiminato)nickel(II). Anal. Sci. 2006, 22, 1221-1226.

533. Xu, W. J.; Chai, Y. Q.; Yuan, R.; Liu, S. L. A novel thiocyanate-selective electrode based on a zinc-phthalocyanine complex. Anal. Bioanal. Chem. 2006, 385, 926-930.

534. Shamsipur, M.; Poursaberi, T.; Rezapour, M.; Ganjali, M. R.; Mousavi, M. F.; Lippolis, V.; Montesu, D. R. [Cu(L)](NO3)(2) (L=4,7-bis(3-aminopropyl)-1-thia-4,7-diazacyclononane) as a suitable ionophore for construction of thiocyanate-selective electrodes and their use in determination of urinary and salivary thiocyanate concentration. Electroanalysis 2004, 16, 13361342.

535. Vlascici, D.; Bizerea, O. S.; Fagadar-Cosma, E. Thiocyanate-selective electrode based on rhodium porphyrin derivates. J. Optoelectron. Adv. M. 2006, 8, 883-887.

536. Segui, M. J.; Lizondo-Sabarter, J.; Martinez-Manez, R.; Sancenon, F.; Soto, J. Linear polyamines as carriers in thiocyanate-selective membrane electrodes. Talanta 2006, 68, 1182-1189.

537. Erden, S.; Demirel, A.; Memon, S.; Yilmaz, M.; Canel, E.; Kilic, E. Using of hydrogen ionselective poly(vinyl chloride) membrane electrode based on calix[4]arene as thiocyanate ionselective electrode. Sens. Actuators B 2006, 113, 290-296. 
538. Dai, J. Y.; Yuan, R.; Chai, Y. Q.; An, L. X.; Zhong, X.; Liu, Y.; Tang, D. P. Highly thiocyanateselective PVC membrane electrode based on lipophilic ferrocene derivative. Electroanalysis 2005, 17, 1865-1869.

539. Chai, Y. Q.; Dai, J. Y.; Yuan, R.; Zhong, X.; Liu, Y.; Tang, D. P. Highly thiocyanate-selective membrane electrodes based on the N,N '-bis-(benzaldehyde)-glycine copper(II) complex as a neutral carrier. Desalination 2005, 180, 207-215.

540. Wang, F. C.; Chai, Y. Q.; Yuan, R.; Chen, C. H.; Dai, J. Y.; Xu, L. A selective membrane electrode for thiocyanate ion based on a bis-taurine-salicylic binuclear copper(II) complex as ionophore. Chin. J. Chem. 2005, 23, 865-869.

541. Xu, L.; Yuan, R.; Chai, Y. Q.; Fu, Y. Z. Novel membrane potentiometric thiocyanate sensor based on tribenzyltin(IV) dithiocarbamate. Electroanalysis 2005, 17, 1003-1007.

542. Shahrokhian, S.; Jannatrezvani, M. J.; Khajehsharifi, H. Rhodium(II) phthalocyanine as a selective carrier in thiocyanate-selective membrane electrode. Anal. Lett. 2005, 38, 1221-1235.

543. Poursaberi, T.; Salavati-Niasari, M.; Khodabakhsh, S.; Hajiagha-Babaei, L.; Shamsipur, M.; Yousefi, M.; Rouhani, S.; Ganjali, M. R. A selective membrane electrode for thiocyanate ion based on a copper-1,8-dimethyl-1,3,6,8,10,13-azacyclotetradecane complex as ionophore. Anal. Lett. 2001, 34, 2621-2632.

544. Shamsipur, M.; Khayatian, G.; Tangestaninejad, S. Thiocyanate-selective membrane electrode based on (octabromotetraphenylporphyrinato)manganese(III) chloride. Electroanalysis 1999, 11, 1340-1344.

545. Amini, M. K.; Shahrokhian, S.; Tangestaninejad, S. Thiocyanate-selective electrodes based on nickel and iron phthalocyanines. Anal. Chim. Acta 1999, 402, 137-143.

546. Dai, J. Y.; Chai, Y. Q.; Yuan, R.; An, L. X.; Liu, Y.; Zhong, X.; Tang, D. P. Tricoordinate Schiff base copper(II) complex as neutral carrier for highly selective thiocyanate electrode. Anal. Lett. 2005, 38, 389-400.

547. Seo, H. R.; Lee, H. K.; Jeon, S. Improved thiocyanate-selective electrode based on tetra(trimethylphenyl)porphyrinato manganese(III) chloride: The electronic and pH effects. Bull. Korean Chem. Soc. 2004, 25, 1484-1488.

548. Buhlmann, P.; Yahya, L.; Enderes, R. Ion-selective electrodes for thiocyanate based on the dinuclear Zinc(II) complex of a Bis-N,O-bidentate Schiff base. Electroanalysis 2004, 16, 973 978.

549. Wang, X. L.; Yuan, R.; Xu, L.; Chai, Y. Q.; Sun, Z. Y.; Huang, X. J.; Li, Q. F.; Zhao, Q. Studies on highly selective thiocyanate electrode based on bisbebzoin-semitriethylenetetraamine binuclear cobalt complex as neutral carriers. Chem. J. Chinese U. 2004, 25, 633-635.

550. Sun, Z. Y.; Yuan, R.; Chai, Y. Q.; Xu, L.; Gan, X. X.; Xu, W. J. Study of a bis-furaldehyde Schiff base copper(II) complex as carrier for preparation of highly selective thiocyanate electrodes. Anal. Bioanal. Chem. 2004, 378, 490-494.

551. Chai, Y. Q.; Sun, Z. Y.; Yuan, R.; Gan, X. X.; Xu, W. J.; Xu, L. Study on highly selective thiocyanate electrode based on benzaldehyde semicarbazone copper(II) complex as neutral carriers. Acta Chim. Sin. 2003, 61, 1511-1515. 
552. Yuan, R.; Wang, X. L.; Xu, L.; Chai, Y. Q.; Sun, Z. Y.; Huang, X. Q.; Li, Q. F.; Zhao, Q.; Zhou, L. Highly selective thiocyanate electrode based on bis-bebzoin-semitriethylenetetraamine binuclear copper(II) complex as neutral carrier. Electrochem. Commun. 2003, 5, 717-721.

553. Khorasani, J. H.; Amini, M. K.; Motaghi, H.; Tangestaninejad, S.; Moghadam, M. Manganese porphyrin derivatives as ionophores for thiocyanate-selective electrodes: the influence of porphyrin substituents and additives on the response properties. Sens. Actuators, B 2002, 87, 448-456.

554. Poursaberi, T.; Salavati-Niasari, M.; Khodabakhsh, S.; Hajiagha-Babaei, L.; Shamsipur, M.; Yousefi, M.; Rouhani, S.; Ganjali, M. R. A selective membrane electrode for thiocyanate ion based on a copper-1,8-dimethyl-1,3,6,8,10,13-azacyclotetradecane complex as ionophore. Anal. Lett. 2001, 34, 2621-2632.

555. Ganjali, M. R. Determination of SCN- in urine and saliva of smokers and non-smokers by SCN-selective polymeric membrane containing a nickel(II)-azamacrocycle complex coated on a graphite electrode. Anal. Sci. 2002, 18, 887-892.

556. Zamani, H. A. Highly selective and sensitive thiocyanate membrane electrode based on nickel(II)-1,4-,8,11,15,18,22,25-octabutoxyphthalocyanine. Anal. Chim. Acta 2006, 555, 336340.

557. Jeon, S. W.; Yeo, H. Y.; Jeong, H. S.; Oh, J. M.; Nam, K. C. Polymeric ISE for hydrogen sulfite based on bis-urea calix[4]diquinones as neutral lipophilic ionophores. Electroanalysis 2003, 15, 872-877.

558. Shamsipur, M.; Yousefi, M.; Hosseini, M.; Ganjali, M. R.; Sharghi, H.; Naeimi, H. A schiff base complex of $\mathrm{Zn}(\mathrm{II})$ as a neutral carrier for highly selective PVC membrane sensors for the sulfate ion. Anal. Chem. 2001, 73, 2869-2874.

559. Ganjali, M. R. Sulfate-selective PVC membrane electrode based on a strontium Schiff's base complex. Ann. Chim (Rome). 2003, 93, 679-690.

560. Ganjali, M. R. A novel potentiometric membrane sensor for quick determination of trace amount of SO42- based on a zinc-Schiff's base. Anal. Lett. 2003, 36, 881-894.

561. Soleymanpour, A.; Asl, E. H.; Nasseri, M. A. Chemically modified carbon paste electrode for determination of sulfate ion by potentiometric method. Electroanalysis 2006, 18, 1598-1064.

562. Ganjali, M. R. Ghorbani, M. Daftari, A. Norouzi, P. Pirelahi, H. Dargahani, H. D. Highly selective liquid membrane sensor based on 1,3,5-triphenylpyrylium perchlorate for quick monitoring of sulfate ions. Bull. Korean Chem. Soc. 2004, 25, 172-176.

563. Nishizawa, S.; Buhlmann, P.; Xiao, K. P.; Umezawa, Y. Application of a bis-thiourea ionophore for an anion selective electrode with a remarkable sulfate selectivity. Anal. Chim. Acta 1998, 358, 35-44.

564. Shamsipur, M.; Yousefi, M.; Ganjali, M. R.; Poursaberi, T.; Faal-Rastgar, M. Highly selective sulfate PVC-membrane electrode based on 2,5-diphenyl-1,2,4,5-tetraaza-bicyclo[2.2.1] heptane as a neutral carrier. Sens. Actuators, B, 2002, 82, 105-110.

565. Ganjali, M. R.; Naji, L.; Poursaberi, T.; Taghizadeh, M.; Pirelahi, H.; Yousefi, M.; YeganehFaal, A.; Shamsipur, M. Novel sulfate ion-selective polymeric membrane electrode based on a derivative of pyrilium perchlorate. Talanta 2002, 58, 359-366. 
566. Ganjali, M. R.; Poujavid, M. R.; Shamsipur, M.; Poursaeri, T.; Rezapour, M.; Javanbakht, M.; Sharghi, H. Novel membrane potentiometric sulfate ion sensor based on zinc-phthalocyanine for the quick determination of trace amounts of sulfate. Anal. Sci. 2003, 19, 995-999.

567. Ganjali, M. R.; Shirvani-Arani, S.; Nourozi, P.; Salimzadeh, D.; Faal-Rastegar, M.; Moghimi, A. A novel sulfate polymeric membrane sensor based on a new bis-pyrylium derivative. Eelectroanalysis 2004, 16, 1009-1013.

568. Ganjali, M. R.; Sepehri, A.; Daftari, A.; Norouzi, P.; Pirelahi, H.; Moradzadegan, A. Determination of salbutamol, amikacin and paromomycin sulfate by a novel sulfate polymeric membrane sensor based on 2,6-diphenyl 4-(4-methoxyphenyl) pyrylium perchlorate. Microchim. Acta 2005, 149, 245-249.

569. Ganjali, M. R.; Mizani, F.; Pirelahi, H.; Taghizadeh, M.; Amani, J. Highly selective and sensitive sulphate membrane sensor based on 2,6-dianisol-4-phenyl-pyrilium perchlorate. Can. J. Anal. Sci. Spect. 2002, 47, 157-163.

570. Mitchell-Koch, J. T.; Malinowska, E.; Meyerhoff, M. E. Gallium(III)-Schiff base complexes as novel ionophores for fluoride selective polymeric membrane electrodes. Electroanalysis 2005, 17, 1347-1353.

571. Chandra, S.; Ruzicka, A.; Svec, P.; Lang, H. Organotin compounds: An ionophore system for fluoride ion recognition. Anal. Chim. Acta 2006, 577, 91-97.

572. Ion, I.; Ion, A. C.; Barbu, L. Potentiometric determination of fluoride in groundwaters. Rev. Roum. Chim. 2005, 50, 407-412.

573. Malinowska, E.; Gorski, L.; Meyerhoff, M. E. Zirconium(IV)-porphyrins as novel ionophores for fluoride-selective polymeric membrane electrodes. Anal. Chim. Acta 2002, 468, 133-141.

574. Ganjali, M. R.; Pourjavid, M. R.; Rezapour, M.; Poursaberi, T.; Daftari, A.; Salavati-Niasari, M. Ruthenium(III) Schiff's base complex as novel chloride selective membrane sensor. Electroanalysis 2004, 16, 922-927.

575. Kumar, K. G.; John, K. S.; Indira, C. J. A chloride ion-selective potentiometric sensor based on a polymeric schiff base complex. Indian J. Chem. Technol. 2006, 13, 13-16.

576. Brasuel, M. G.; Miller, T. J.; Kopelman, R.; Philbert, M. A. Liquid polymer nano-PEBBLEs for Cl- analysis and biological applications. Analyst 2003, 128, 1262-1267.

577. Soleymanpour, A.; Asl, E. H.; Nabavizadeh, S. M. Perchlorate selective membrane electrodes based on synthesized platinum(II) complexes for low-level concentration measurements. Sens. Actuators B 2007, 120, 447-454.

578. Segui, M. J.; Lizondo-Sabater, J.; Martinez-Manez, R.; Sancenon, F.; Soto, J.; Garcia-Breijo, E.; Gil, L. An ion-selective electrode for anion perchlorate in thick-film technology. Sensors 2006, 6, 480-491.

579. DiBenedetto, L. T.; Dimitrakopoulos, T.; Farrell, J. R.; Iles, P. J. Evaluation of perchlorate tolerant photo-cured calcium selective electrodes for use in flow injection potentiometry. Talanta 1997, 44, 349-356.

580. Errachid, A.; Perez-Jimenez, C.; Casabo, J.; Escriche, L.; Munoz, J. A.; Bratov, A.; Bausells, J. Perchlorate-selective MEMFETs and ISEs based on a new phosphadithiamacrocycle. Sens. Actuators B 1997, 43, 206-210. 
581. Perez-Olmos, R.; Rios, A.; Martin, M. P.; Lapa, R. A. S.; Lima, J. Construction and evaluation of ion selective electrodes for perchlorate with a summing operational amplifier: application to pyrotechnics mixtures analysis. Analyst 1999, 124, 97-100.

582. Ardakani, M. M.; Jalayer, M.; Naeimi, H.; Zare, H. R.; Moradi, L. Perchlorate-selective membrane electrode based on a new complex of uranil. Anal. Bioanal. Chem. 2005, 381, 11861192.

583. Shamsipur, M.; Soleymanpour, A.; Akhond, M.; Sharghi, H.; Hasaninejad, A. R. Perchlorate selective membrane electrodes based on a phosphorus(V)-tetraphenylporphyrin complex. Sens. Actuators B 2003, 89, 9-14.

584. Sanchez-Pedreno, C.; Ortuno, J. A.; Hernandez, J. Perchlorate-selective polymeric membrane electrode based on a gold(I) complex: application to water and urine analysis. Anal. Chim. Acta 2000, 415, 159-164.

585. Ganjali, M. R.; Norouzi, P.; Faridbod, F.; Yousefi, M.; Naji, L.; Salavati-Niasari, M. Perchlorateselective membrane sensors based on two nickel-hexaazamacrocycle complexes. Sens. Actuators B 2007, 120, 494-499.

586. Ganjali, M. R.; Yousefi, M.; Poursaberi, T.; Naji, L.; Salavati-Niasari, M.; Shamsipur, M. Highly selective and sensitive perchlorate sensors based on some recently synthesized $\mathrm{Ni}(\mathrm{II})$ hexaazacyclotetradecane complexes. Electroanalysis 2003, 15, 1476-1480.

587. Bendikov, T. A.; Harmon, T. C. Long-lived solid state perchlorate ion selective sensor based on doped poly(3,4-ethylenedioxythiophene) (PEDOT) films. Anal. Chim. Acta 2005, 551, 30-36.

588. Ganjali, M.R. Norouzi, P. Golmohammadi, M. Rezapour, M. and Salavati-Niasari, M. Novel bromide PVC-based membrane sensor based on iron(III)-salen. Electroanalysis 2004, 16, 910 914.

589. Singh, A. K.; Mehtab, S.; Saxena, P. A bromide selective polymeric membrane electrode based on Zn(II) macrocyclic complex. Talanta 2006, 69, 1143-1148.

590. Shamsipur, M.; Ershad, S.; Samadi, N.; Moghimi, A.; Aghabozorg, H. A novel chemically modified carbon paste electrode based on a new mercury(II) complex for selective potentiometric determination of bromide ion. J. Solid State Electrochem. 2005, 9, 788-793.

591. Ganjali, M. R.; Tahami, M.; Poursaberi, T.; Pazoukian, A. R.; Javanbakht, M.; Shamsipur, M.; Baezat, M. R. Novel bromide liquid membrane electrode. Anal. Lett. 2003, 36, 347-360.

592. Shamsipur, M.; Rouhani, S.; Mohajeri, A.; Ganjali, M. R.; Rashidi-Ranjbar, P. A bromide ionselective polymeric membrane electrode based on a benzo-derivative xanthenium bromide salt. Anal. Chim. Acta 2000, 418, 197-203.

593. Shamsipur, M.; Sadeghi, S.; Naeimi, H.; Sharghi, H. Iodide ion-selective PVC membrane electrode based on a recently synthesized salen-Mn(II) complex. Pol. J. Chem. 2000, 74, 231238.

594. Shamsipur, M.; Soleymanpour, A.; Akhond, M.; Sharghi, H.; Naseri, M. A. Iodide-selective carbon paste electrodes based on recently synthesized Schiff base complexes of Fe(III). Anal. Chim. Acta 2001, 450, 37-44. 
595. Ganjali, M.R. Poursaberi, T. Hosseini, M. Salavati-Niasari, M. Yousefi, M.; Shamsipur, M. Highly selective iodide membrane electrode based on a cerium salen. Anal. Sci. 2002, 18, 289292.

596. Farhadi, K.; Maleki, R.; Yamchi, R.H.; Sharghi, H.; Shamsipur, M. [Tetrakis(4-N,Ndimethylaminobenzene)porphyrinato]-manganese(III) acetate as a novel carrier for a selective iodide PVC membrane electrode. Anal. Sci. 2004, 20, 805-809.

597. Zare, H. R.; Memarzadeh, F.; Gorji, A.; Ardakani, M. M. Iodide-selective membrane electrode based on salophen complex of cobalt (III). J. Braz. Chem. Soc. 2005, 16, 571-577.

598. Abdel-Latif, M. S.; Al-Saraj, M. R. A novel potentiometric solid-state iodide sensor. Anal. Lett. 2007, 40, 729-736.

599. Wygladacz, K.; Bakker, E. Fluorescent microsphere fiber optic microsensor array for direct iodide detection at low picomolar concentrations. Analyst 2007, 132, 268-272.

600. Jeong, D. C.; Lee, H. K.; Jeon, S. Polymeric iodide-ion selective electrodes based on urea derivative as an ionophore. Bull. Korean Chem. Soc. 2006, 27, 1985-1988.

601. Sun, C. Q.; Zhao, J. H.; Xu, H. D.; Sun, Y. P.; Zhang, X.; Shen, J. C. Fabrication of a multilayer film electrode containing porphyrin and its application as a potentiometric sensor of iodide ion. Talanta 1998, 46, 15-21.

602. Ganjali, M.R. Daftari A, Mizani, F. and Salavati-Niasari, M. Titanium, acetylacetonate as an excellent ion-carrier in construction of iodide sensor. Bull. Korean Chem. Soc. 2003, 24, 23-26.

603. Almeida, A. A.; Jun, X.; Lima, J. Ion chromatographic determination of iodide in urine and serum using a tubular ion-selective electrode based on a homogeneous crystalline membrane. Mikrochim. Acta 1997, 127, 55-60.

604. Poursaberi, T. Hosseini, M. Taghizadeh, M. Pirelahi, H. Shamsipur, M.; Ganjali, M.R. A selective membrane electrode for iodide ion based on a thiopyrilium ion derivative as a new ionophore. Microchem. J. 2002, 72, 77-83.

605. Zeng, Y. L.; Tang, C. R.; Sheng, G. L.; Yu, R. Q. Studies on liquid membrane iodide selective electrode with long lifetime and high selectivity. Chem. J. 1999, 20, 603-607.

606. Katsu, T.; Hashimoto, T. Triphenyllead chloride as a new ionophore for an iodide-selective membrane electrode. Anal. Lett. 1999, 32, 665-675.

607. Chai, Y. Q.; Yuan, R.; Xu, L.; Xu, W. J.; Dai, J. Y.; Jiang, F. A highly sensitive PVC membrane iodide electrode based on complexes of mercury(II) as neutral carrier. Anal. Bioanal. Chem. 2004, 380, 333-338.

608. Shahrokhian, S.; Taghani, A.; Moattar, F. Iodide-selective electrode based on copper phthalocyanine. Electroanalysis 2002, 14, 1621-1628.

609. Lizondo-Sabater, J.; Martinez-Manez, R.; Sancenon, F.; Segui, M. J.; Soto, J. Cobalt(II) and nickel(II) complexes of a cyclam derivative as carriers in iodide-selective electrodes. Anal. Chim. Acta 2002, 459, 229-234.

610. Dobcnik, D.; Kolar, M.; Komljenovic, J.; Radic, N. Preparation of an ion-selective electrode by chemical treatment of copper wire for the measurement of copper(II) and iodide by batch and flow-injection potentiometry. Fresen. J. Anal. Chem. 1999, 365, 314-319. 
611. Sadeghi, S.; Gafarzadeh, A.; Naseri, M. A.; Sharghi, H. Triiodide-selective polymeric membrane electrodes based on Schiff base complexes of $\mathrm{Cu}(\mathrm{II})$ and Fe(III). Sens. Actuators B 2004, 98, 174179.

612. Ganjali, M. R.; Norouzi, P.; Shirvani-Arani, S.; Salavati-Niasari, M. Novel triiodide PVC-based membrane sensor based on a charge transfer complex of iodine and bis(2hydroxyacetophenone)butane-2,3-dihydrazone. Bull. Korean Chem. Soc. 2005, 26, 1738-1742.

613. Ganjali, M. R.; Emami, M.; Javanbakht, M.; Salavati-Niasari, M.; Shamsipur, M.; Yousefi, M. Novel triiodide ion-selective polymeric membrane sensor based on mercury-salen. Sens. Actuators B 2005, 105, 127-131.

614. Sadeghi, S.; Fathi, F.; Ali Esmaeili, A.; Naeimi, H. Novel triiodide ion-selective polymeric membrane electrodes based on some transition metal-Schiff base complexes. Sens. Actuators $B$ 2006, 114, 928-935.

615. Ganjali, M. R.; Norouzi, P.; Qomi, M.; Salavati-Niasari, M. Charge-transfer complex between iodine and a new Schiff's base as anion-carrier in construction of a highly selective triiodide PVC-based membrane electrode. Can. J. Anal. Sci. Spect. 2006, 51, 108-116.

616. Ganjali, M. R.; Shirvani-Arani, S.; Bidhendi, G. N.; Norouzi, P.; Salavati-Niasari, M. Highly selective and sensitive triiodide PVC-membrane electrode based on a new charge-transfer complex of bis(2,4-dimethoxybenzaidehyde)butane-2,3-dihydrazone with iodine. J. Chin. Chem. Soc. 2006, 53, 275-281.

617. Ganjali, M. R.; Norouzi, P.; Shirvani-Arani, S.; Kakanezhadifard, A. Synthesis of a chargetransfer complex of (1,3-diphenyldihydro-1H-imidazole)-4,5-dione dioxide with iodide and its application to the development of a highly selective and sensitive triiodide PVC-membrane electrode. J, Anal. Chem. 2007, 62, 279-284.

618. Sadeghi, S.; Fathi, F.; Ali Esmaeili, A.; Naeimi, H. Novel triiodide ion-selective polymeric membrane electrodes based on some transition metal-Schiff base complexes. Sens. Actuators $B$ 2006, 114, 928-935.

619. Ganjali, M. R.; Norouzi, P.; Shirvani-Arani, S.; Salavati-Niasari, M. Novel triiodide PVC-based membrane sensor based on a charge transfer complex of iodine and bis(2hydroxyacetophenone)butane-2,3-dihydrazone. Bull. Korean Chem. Soc. 2005, 26, 1738-1742.

620. Khayatian, G.; Rezatabar, H.; Salimi, A. Charge-transfer triiodide ion-selective electrode based on 7,16-dibenzyl-1,4,10,13-tetraoxa-7,16-diazacyclooctadecane. Anal. Sci. 2005, 21, 297-302.

621. Rouhollahi, A.; Shamsipur, M. Triiodide PVC membrane electrode based on a charge-transfer complex of iodine with 2,4,6,8-tetraphenyl-2,4,6,8-tetraazabicyclo[3.3.0]octane. Anal. Chem. 1999, 71, 1350-1353.

622. Ortuno, J. A.; Sanchez-Pedreno, C.; Hernandez, J.; Oliva, D. J. Flow-injection potentiometric determination of triiodide by plasticized poly(vinyl chloride) membrane electrodes and its application to the determination of chlorine-containing disinfectants. Talanta 2005, 65, 11901195.

623. Ganjali, M. R.; Emami, M.; Javanbakht, M.; Salavati-Niasari, M.; Shamsipur, M.; Yousefi, M. Novel triiodide ion-selective polymeric membrane sensor based on mercury-salen. Sens. Actuators B 2005, 105, 127-131. 
624. Farhadi, K.; Maleki, R. Construction of triiodide ion selective electrodes based on phenothiazine derivatives. Anal. Lett. 2004, 37, 1063-1078.

625. Farhadi, K.; Shaikhlouei, H.; Maleki, R.; Sharghi, H.; Shamsipur, M. Highly selective triiodide polymeric membrane electrode based on tetra(p-chlorophenyl)porphyrinato manganese (III) acetate. Bull. Korean Chem. Soc, 2002, 23, 1635-1639.

626. Farhadi, K.; Bonab, H. S.; Maleki, R.; Shamsipur, M.; Shargi, H. Tetrachlorophenylporphyrinato manganese(III) acetate as a new ionophore for a coated triiodide ion-selective electrode. J. Chin. Chem. Soc. 2002, 49, 861-866.

627. Farhadi, K.; Maleki, R.; Shamsipur, M. Triiodide ion-selective polymeric membrane electrode based on a ketoconazole-triiodide ion pair. Electroanalysis 2002, 14, 760-766.

628. Sadeghi, S.; Dashti, G. R. Triiodide PVC membrane electrodes based on charge-transfer complexes. Anal. Chem. 2002, 74, 2591-2595.

629. Farhadi, K.; Maleki, R. Clotrimazole-triiodide ion association as an ion exchanger for a triiodide ion-selective electrode. Anal. Sci. 2002, 18, 133-136.

630. Ganjali, M. R.; Norouzi, P.; Shirvani-Arani, S.; Salavati-Niasari, M. Novel triiodide PVC-based membrane sensor based on a charge transfer complex of iodine and bis(2hydroxyacetophenone)butane-2,3-dihydrazone. Bull. Korean Chem. Soc. 2005, 26, 1738-1742.

631. Montenegro, M.; Sales, M. G. F. Flow-injection analysis of dopamine in injections with a periodate-selective electrode. J. Pharm. Sci. 2000, 89, 876-884.

632. Metelka, R.; Slavikova, S.; Vytras, K. Determination of arsenate and organic arsenic via potentiometric titration of its heteropoly anions. Talanta 2002, 58, 147-151.

(C) 2008 by MDPI (http://www.mdpi.org). Reproduction is permitted for noncommercial purposes. 